\title{
3D-HST WFC3-SELECTED PHOTOMETRIC CATALOGS IN THE FIVE CANDELS/3D-HST FIELDS: PHOTOMETRY, PHOTOMETRIC REDSHIFTS, AND STELLAR MASSES
}

\author{
Rosalind E. Skelton ${ }^{1,2}$, Katherine E. Whitaker ${ }^{3}$, Ivelina G. Momcheva ${ }^{2}$, Gabriel B. Brammer ${ }^{4}$, \\ Pieter G. van Dokkum ${ }^{2}$, Ivo Labbé ${ }^{5}$, Marijn Franx ${ }^{5}$, Arjen van der Wel ${ }^{6}$, Rachel Bezanson ${ }^{2,7}$, Elisabete Da Cunha ${ }^{6}$,

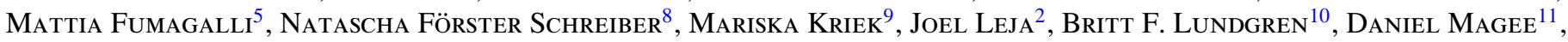 \\ Danilo Marchesini ${ }^{12}$, Michael V. Maseda ${ }^{6}$, Erica J. Nelson ${ }^{2}$, Pascal Oesch $^{2}$, Camilla Pacifici $^{13}$, Shannon G. Patel $^{14}$, \\ Sedona Price ${ }^{9}$, Hans-Walter Rix ${ }^{6}$, Tomer Tal ${ }^{11}$, David A. Wake ${ }^{10,15}$, and Stijn Wuyts ${ }^{8}$ \\ ${ }^{1}$ South African Astronomical Observatory, PO Box 9, Observatory, Cape Town 7935, South Africa; ros@ saao.ac.za \\ ${ }^{2}$ Department of Astronomy, Yale University, 260 Whitney Avenue, New Haven, CT 06511, USA \\ ${ }^{3}$ Astrophysics Science Division, Goddard Space Flight Center, Greenbelt, MD 20771, USA \\ ${ }^{4}$ Space Telescope Science Institute, 3700 San Martin Drive, Baltimore, MD 21218, USA \\ ${ }^{5}$ Leiden Observatory, Leiden University, Leiden, The Netherlands \\ ${ }^{6}$ Max Planck Institute for Astronomy (MPIA), Königstuhl 17, D-69117, Heidelberg, Germany \\ ${ }^{7}$ Steward Observatory, University of Arizona, 933 North Cherry Avenue, Tucson, AZ 85721, USA \\ ${ }^{8}$ Max-Planck-Institut für extraterrestrische Physik, Giessenbachstrasse, D-85748 Garching, Germany \\ ${ }^{9}$ Astronomy Department, University of California, Berkeley, CA 94720, USA \\ ${ }^{10}$ Department of Astronomy, University of Wisconsin-Madison, 475 North Charter Street, Madison, WI 53706, USA \\ ${ }^{11}$ Department of Astronomy \& Astrophysics, University of California, Santa Cruz, CA, USA \\ ${ }_{12}$ Department of Physics and Astronomy, Tufts University, Medford, MA 02155, USA \\ ${ }^{13}$ Yonsei University Observatory, Yonsei University, Seoul 120-749, Republic of Korea \\ ${ }^{14}$ Carnegie Observatories, Pasadena, CA 91101, USA \\ ${ }^{15}$ Department of Physical Sciences, The Open University, Milton Keynes, MK7 6AA, UK \\ Received 2014 March 14; accepted 2014 July 10; published 2014 October 1
}

\begin{abstract}
The 3D-HST and CANDELS programs have provided WFC3 and ACS spectroscopy and photometry over $\approx 900 \mathrm{arcmin}^{2}$ in five fields: AEGIS, COSMOS, GOODS-North, GOODS-South, and the UKIDSS UDS field. All these fields have a wealth of publicly available imaging data sets in addition to the Hubble Space Telescope (HST) data, which makes it possible to construct the spectral energy distributions (SEDs) of objects over a wide wavelength range. In this paper we describe a photometric analysis of the CANDELS and 3D-HST HST imaging and the ancillary imaging data at wavelengths $0.3-8 \mu \mathrm{m}$. Objects were selected in the WFC 3 near-IR bands, and their SEDs were determined by carefully taking the effects of the point-spread function in each observation into account. A total of 147 distinct imaging data sets were used in the analysis. The photometry is made available in the form of six catalogs: one for each field, as well as a master catalog containing all objects in the entire survey. We also provide derived data products: photometric redshifts, determined with the EAZY code, and stellar population parameters determined with the FAST code. We make all the imaging data that were used in the analysis available, including our reductions of the WFC3 imaging in all five fields. 3D-HST is a spectroscopic survey with the WFC3 and ACS grisms, and the photometric catalogs presented here constitute a necessary first step in the analysis of these grism data. All the data presented in this paper are available through the 3D-HST Web site (http://3dhst.research.yale.edu).
\end{abstract}

Key words: catalogs - galaxies: evolution - galaxies: general - methods: data analysis - techniques: photometric

Online-only material: color figures

\section{INTRODUCTION}

Large multi-wavelength photometric surveys have made it possible to study galaxy populations over most of cosmic history. Near-infrared selected samples have been used to trace the evolution of the stellar mass function (e.g., Marchesini et al. 2009; Pérez-González et al. 2008), the star-formation-mass relation (e.g., Whitaker et al. 2012), and the structural evolution of galaxies (e.g., Franx et al. 2008; Bell et al. 2012; Wuyts et al. 2012; van der Wel et al. 2012). Until recently most of these surveys relied on deep, wide-field imaging from ground-based telescopes (e.g., Muzzin et al. 2013; Williams et al. 2009). The WFC3 camera on the Hubble Space Telescope (HST) has opened up the possibility to select and study galaxies at near-infrared wavelengths with excellent sensitivity and spatial resolution. Furthermore, the WFC3 grisms enable space-based near-IR slitless spectroscopy of all objects in the camera's field-of-view (see, e.g., van Dokkum \& Brammer 2010).
The largest area WFC3 imaging survey done to date is the Cosmic Assembly Near-infrared Deep Extragalactic Legacy Survey (CANDELS; Grogin et al. 2011; Koekemoer et al. 2011), a 912 orbit Multi-Cycle Treasury imaging program (PIs: $\mathrm{S}$. Faber and H. Ferguson). This survey encompasses five wellstudied extragalactic fields: the All-wavelength Extended Groth Strip International Survey (AEGIS) field, the Cosmic Evolution Survey (COSMOS) field, the Great Observatories Origins Survey (GOODS) Northern and Southern fields (GOODS-North and GOODS-South) and the UKIRT InfraRed Deep Sky Surveys (UKIDSS) Ultra Deep Field (UDS). The coordinates of the five fields are given in Table 1. As these fields have been observed extensively over the past decade, the CANDELS imaging builds on a vast array of publicly available photometry at other wavelengths, ranging from the near-UV to the far-IR (see Grogin et al. 2011).

Building, in turn, on the CANDELS survey, we have undertaken a WFC3 spectroscopic survey in these same fields. 

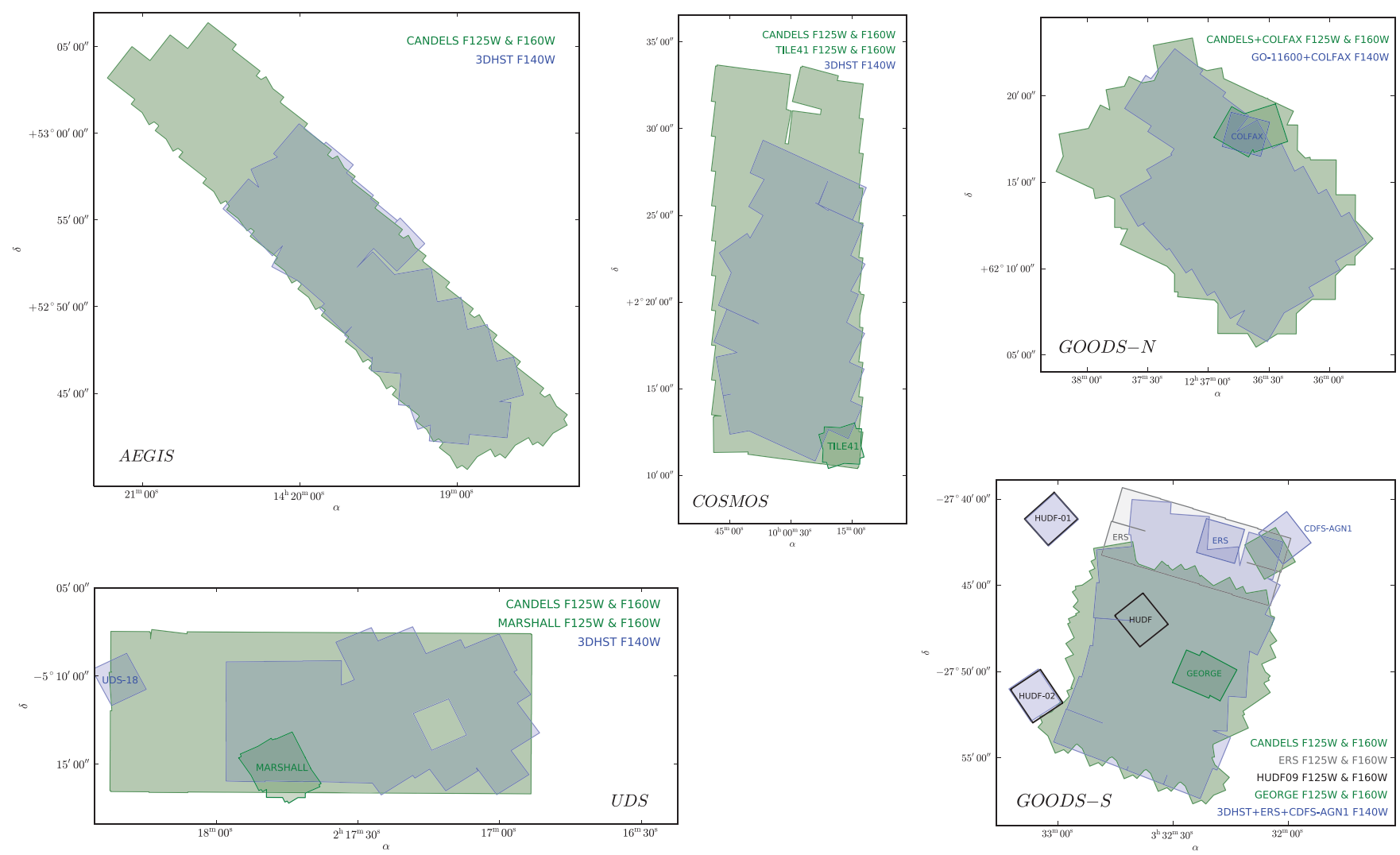

Figure 1. Layout of the WFC3 observations used in this paper. The catalogs presented in this paper cover the entire area that is covered by either $F 125 W, F 140 W$, or $F 160 \mathrm{~W}$, in each of the five fields. Table 2 lists the programs and PIs for all the HST/WFC3 observations that were used in our work. North is up and east is to the left. (A color version of this figure is available in the online journal.)

Table 1

3D-HST Fields

\begin{tabular}{lcccc}
\hline \hline Field & $\begin{array}{c}\text { R.A. } \\
(\mathrm{h} \mathrm{m} \mathrm{s})\end{array}$ & $\begin{array}{c}\text { Decl. } \\
(\mathrm{d} \mathrm{m} \mathrm{s})\end{array}$ & $\begin{array}{c}\text { Total Area } \\
\left(\operatorname{arcmin}^{2}\right)\end{array}$ & $\begin{array}{c}\text { Science Area } \\
\left(\operatorname{arcmin}^{2}\right)\end{array}$ \\
\hline AEGIS & 141836.00 & +52390.00 & 201 & 192.4 \\
COSMOS & 100031.00 & +02240.00 & 199 & 183.9 \\
GOODS-North & 123554.98 & +621151.3 & 164 & 157.8 \\
GOODS-South & 033230.00 & -274719.00 & 177 & 171.0 \\
UDS & 021749.00 & -05122.00 & 201 & 191.2 \\
\hline
\end{tabular}

3D-HST is a 248-orbit HST Treasury program (Programs 12177 and 12328; PI: P. van Dokkum) that uses the WFC3 G141 grism for slitless spectroscopy across $\sim 700 \mathrm{arcmin}^{2}$ of the sky, approximately $75 \%$ of the CANDELS area (see Figure 1). This rich data set is providing excellent redshifts and spatially resolved spectral lines for thousands of galaxies in the key epoch $1<z<3$ (e.g., Whitaker et al. 2013; Nelson et al. 2012; Brammer et al. 2012a). The survey is described in Brammer et al. (2012b). We targeted four of the five CANDELS fields: AEGIS, COSMOS, GOODS-South, and UDS. WFC3/G141 grism data in GOODSNorth were already available from program GO-11600 (PI: B. Weiner); these data are incorporated in the 3D-HST analysis and data releases. The 3D-HST observations yield the following four types of data: WFC3 G141 grism observations, WFC3 F140W imaging for wavelength calibration of the spectra, parallel ACS G800L grism spectroscopy, and parallel $F 814 W$ imaging.

The scientific returns from CANDELS, 3D-HST, and all other surveys in these five fields are maximized when the various data sets are combined in a homogeneous way, as it is often the combination of different kinds of data that provides new insight. To give just one example, Wuyts et al. (2012) studied the structure of galaxies (determined from HST imaging) as a function of photometric redshift (determined from fits to multiwavelength, broadband spectral energy distributions, SEDs) and star formation rate (determined from SEDs and space-based infrared photometry). The interpretation of the data is also made easier when all information is used: it is much easier to correctly identify an emission line in a grism spectrum when the redshift range of the object is constrained by the available photometric information.

The 3D-HST project has the aim of providing this homogenous combination of data sets in the five CANDELS/3D-HST fields. This undertaking has several linked aspects.

1. We obtained and reduced the available HST/WFC3 imaging in the fields, using the same pixel scale and tangent point as those used by the CANDELS team. The WFC3 imaging includes the CANDELS data and also the Early Release Science data in GOODS-South and various other programs such as the HUDF09 Ultra Deep Field campaign.

2. Source catalogs are created with SExtractor (Bertin \& Arnouts 1996), detecting objects in deep combined $F 125 W+F 140 W+F 160 W$ images.

3. These source catalogs, along with the detection images, associated segmentation maps and PSFs, are used as the basis to measure photometric fluxes at wavelengths $0.3-8 \mu \mathrm{m}$ from a large array of publicly available imaging data sets. The resulting SEDs are of very high quality, particularly in fields with extensive optical and near-IR medium band photometry.

4. Photometric redshifts, and redshift probability distributions, are estimated from the SEDs. 
5. Stellar population parameters are determined by fitting stellar population synthesis models to the SEDs, using the photometric redshifts as input.

6. Mid- and far-IR photometry is obtained from Spitzer/MIPS and Herschel imaging. These data, combined with restframe UV emission measurements from the SEDs, are used to determine star formation rates of the galaxies.

7. The set of images, PSFs, and catalogs is used to measure structural parameters of the objects in the WFC3 and ACS bands, following the methodology of van der Wel et al. (2012).

8. The coordinates in the catalogs and segmentation maps are mapped back to the original (interlaced) coordinate system of the WFC3 and ACS grism data, and spectra are extracted for each object in the photometric catalog that is covered by the grism. No source matching is required since each extracted spectrum is associated with a particular object in the photometric catalog. The photometric SED can be combined directly with the grism spectroscopy of each object for further analysis.

9. The spectra and SEDs are fitted simultaneously, to measure redshifts and emission line fluxes.

10. Parameters measured in steps 5-7 are re-measured using the updated redshifts.

In this paper we describe steps $1-5$ of the 3D-HST project; steps 6-10 will be described in future papers. As outlined above the photometric catalogs ultimately serve as input to the fits of the grism spectroscopy, but as we show here they constitute a formidable data set in their own right. Furthermore, the majority of objects in the photometric catalogs are so faint that the grism does not provide useful additional information. We provide the homogenized set of imaging data sets that are used in this paper to the community, as well as the photometric catalogs and the EAZY and FAST fits to the photometry. The structure of this paper is as follows. In Section 2.1, we describe the data reduction and mosaicking of the WFC3 detection images. Section 2.2 details the additional multi-wavelength data available for each field. Section 3 describes our photometric methods, accounting for differences in the depth and resolution of the data in different bands. We discuss the survey completeness in Section 3.10. We verify the quality and consistency of the catalogs in Section 4. In Sections 5, 5.3, and 5.4 we describe the photometric redshift, rest-frame color, and stellar population parameter fits to the SEDs. Additional information on the PSFs and zero point offsets applied to the catalogs are provided in Appendices A and B. We present a comparison of our photometry with other available catalogs for each of the five fields in Appendix C.

We use the AB magnitude system throughout (Oke 1971) and where necessary, a $\Lambda$ CDM cosmology with $\Omega_{M}=0.3, \Omega_{\Lambda}=$ 0.7 and $H_{0}=70 \mathrm{~km} \mathrm{~s}^{-1} \mathrm{Mpc}^{-1}$.

\section{DATA SETS}

The five CANDELS/3D-HST fields have been observed with HST/WFC3, HST/ACS, Spitzer, and many ground-based telescopes. In each field, the heart of the data consists of the WFC $3 F 125 W, F 140 W$, and $F 160 W$ images obtained by the CANDELS and 3D-HST Treasury programs. In this section, we describe our reductions of these data, and briefly discuss all other space- and ground-based data that are used to construct the SEDs.

The photometric catalogs make use of some 150 different image mosaics. As part of the analysis we projected these data onto our astrometric grid and pixel scale (which is identical to that used by the CANDELS team) and, in some cases, process the images to remove artifacts. For convenience, all images used in our work and the derived photometry are made available for download on the 3D-HST Web site. The images are also available through the Mikulski Archive for Space Telescopes (MAST). The majority of these data have been made public previously, but this is the first time all of them are offered as one comprehensive data set.

\subsection{WFC3 Imaging \\ 2.1.1. Sources of Data}

The majority of HST/WFC3 imaging comes from the 3DHST and CANDELS surveys which, jointly, have covered $\sim 940 \mathrm{arcmin}^{2}$ in three infrared filters: $F 125 \mathrm{~W}, F 140 \mathrm{~W}$, and $F 160 \mathrm{~W}$. The coordinates of the five fields and the areas covered by the WFC3 imaging are given in Table 1. The "Total Area" column indicates the total area for which there is data in $F 125 W, F 140 W$, or $F 160 W$, while the "Science Area" column indicates the useful area after accounting for bright stars and regions without sufficient coverage in one of the CANDELS bands (see Section 3.11). Other HST programs have carried out observations of portions of these fields with combinations of the three filters. In order to increase the depth of the data and maximize the footprint of the mosaics we have incorporated many of these additional data sets. Table 2 lists all HST/WFC3 data sets used in our work as well as the HST proposal ID which requested the observations, the proposal PI, and the total number of orbits. In total, we utilize 1160 orbits of $H S T /$ WFC3 imaging observations. Figure 1 illustrates the layout of the WFC3 observations. We summarize the relevant observational details for each field below, focusing primarily on the CANDELS and 3D-HST data. Details for the remaining programs can be found on the MAST archive. ${ }^{16}$

All near-infrared HST observations were obtained using the WFC3 IR detector (WFC3/IR) which has a $1024 \times 1024$ $\mathrm{HgCdTe}$ array. The usable portion of the detector is $1014 \times$ 1014 pixels, covering a region of $136^{\prime \prime} \times 123^{\prime \prime}$ across with a native pixel scale of $0^{\prime \prime} 128$ pixel $^{-1}$ (at the central reference pixel). The majority of currently available observations in the five deep fields are done in three wide filters: $F 125 \mathrm{~W}$, $F 140 \mathrm{~W}$, and $F 160 \mathrm{~W}$, which cover the wavelength ranges of $\sim 1.1 \mu \mathrm{m}-1.4 \mu \mathrm{m}, \sim 1.2 \mu \mathrm{m}-1.6 \mu \mathrm{m}$, and $1.4 \mu \mathrm{m}-1.7 \mu \mathrm{m}$, respectively. The $F 125 W$ filter is slightly wider than the standard ground-based $J$ band, while the $F 160 W$ is slightly narrower to better match the detector QE and to limit the effects of the thermal background. The $F 140 \mathrm{~W}$ filter covers the gap between the $J$ and $H$ bands which is inaccessible from the ground. The standard designations for the three filters are $J_{F 125 W}, J H_{F 140 W}$ and $H_{F 160 W}$, however, throughout this paper we will refer to them by the filter name to avoid confusion with ground-based $J$ and $H$ bandpasses. The WFC3/IR PSF has an FWHM between $0{ }^{\prime \prime} 13$ and $0^{\prime \prime} 15$ over this wavelength range (1.02-1.18 native pixels).

3D-HST is primarily a spectroscopic survey, with most of the $2 \times 248$ primary and parallel orbits devoted to grism observations (see, e.g., Brammer et al. 2012b). In addition to the grism exposures, we obtain direct images in broadband filters, as required for wavelength calibration of the spectra and for associating spectra with objects (Kümmel et al. 2009).

\footnotetext{
16 http://archive.stsci.edu/hst/search.php
} 
Table 2

HST Observations

\begin{tabular}{|c|c|c|c|c|c|c|c|}
\hline Field & Instrument & Filters & $N_{\text {orbits }}$ & Proposal ID & HST Cycle & Survey & PI \\
\hline \multirow[t]{3}{*}{ AEGIS } & WFC3 & $F 125 W, F 160 W$ & 90 & 12063 & 18 & CANDELS & Faber \\
\hline & WFC3 & $F 140 W$ & $8^{a}$ & 12177 & 18 & 3D-HST & van Dokkum \\
\hline & $\mathrm{ACS}$ & $F 606 W, F 814 W$ & 90 & 12063 & 18 & CANDELS & Faber \\
\hline \multirow[t]{4}{*}{ COSMOS } & WFC3 & $F 125 W, F 160 W$ & 88 & 12440 & 18 & CANDELS & Faber \\
\hline & WFC3 & $F 125 W, F 160 W$ & $10^{\mathrm{a}}$ & 12461 & 19 & TILE41 & Riess \\
\hline & WFC3 & $F 140 W$ & $8^{\mathrm{a}}$ & 12328 & 18 & 3D-HST & van Dokkum \\
\hline & $\mathrm{ACS}$ & $F 606 W, F 814 W$ & 88 & 12440 & 18 & CANDELS & Faber \\
\hline \multirow[t]{5}{*}{ GOODS-N } & WFC3 & $F 125 W, F 160 W$ & 173 & $12443-12445$ & 19 & CANDELS & Faber \\
\hline & WFC3 & $F 140 W$ & $7^{\mathrm{a}}$ & 11600 & 17 & AGHAST & Weiner \\
\hline & WFC3 & $F 125 W, F 140 W, F 160 W$ & $5^{\mathrm{a}}$ & 12461 & 19 & COLFAX & Riess \\
\hline & ACS & $F 606 W, F 814 W, F 850 L P$ & 219 & $12442-12445$ & 19 & CANDELS & Faber \\
\hline & $\mathrm{ACS}$ & $F 435 W, F 606 W, F 775 W, F 850 W$ & 199 & 9583 & 11 & GOODS & Giavalisco \\
\hline \multirow[t]{8}{*}{ GOODS-S } & WFC3 & $F 125 W, F 160 W$ & 173 & 12061,12062 & 18 & CANDELS & Faber \\
\hline & WFC3 & $F 140 W$ & $11^{\mathrm{a}}$ & 12177 & 18 & 3D-HST & van Dokkum \\
\hline & WFC3 & $F 125 W, F 160 W$ & $4^{\mathrm{a}}$ & 12099 & 18 & GEORGE & Riess \\
\hline & WFC3 & $F 140 W$ & $1^{\mathrm{a}}$ & 12190 & 18 & CDFS-AGN1 & Koekemoer \\
\hline & WFC3 & $F 125 W, F 140 W, F 160 W$ & 12 & 11359,11360 & 17 & ERS & O’Connell \\
\hline & WFC3 & $F 125 W, F 160 W$ & 149 & 11563 & 17 & HUDF09 & Illingworth \\
\hline & ACS & $F 606 W, F 814 W, F 850 L P$ & 229 & $12060-12062$ & 18 & CANDELS & Faber \\
\hline & $\mathrm{ACS}$ & $F 435 W, F 606 W, F 775 W, F 850 W$ & 199 & 9425 & 11 & GOODS & Giavalisco \\
\hline \multirow[t]{4}{*}{ UDS } & WFC3 & $F 125 W, F 160 W$ & 88 & 12064 & 18 & CANDELS & Faber \\
\hline & WFC3 & $F 140 W$ & $8^{\mathrm{a}}$ & 12328 & 18 & 3D-HST & van Dokkum \\
\hline & WFC3 & $F 125 W, F 160 W$ & $18^{\mathrm{a}}$ & 12099 & 18 & MARSHALL & Riess \\
\hline & ACS & $F 606 W, F 814 W$ & 88 & 12064 & 18 & CANDELS & Faber \\
\hline
\end{tabular}

Note. ${ }^{a}$ For orbits that contain grism observations, the number of orbits has been determined based on the fraction of the time dedicated to direct images and rounded to the nearest full orbit.

These direct images are obtained in the $F 814 W$ filter for ACS and in the $F 140 W$ filter for WFC3. The $F 140 W$ filter is broad and overlaps with most of the wavelength range of the G141 grism. In the context of the available imaging in the CANDELS fields, the $F 140 W$ data offer an important photometric datapoint between the CANDELS $F 125 W$ and $F 160 \mathrm{~W}$ imaging described below. For the current photometric catalogs, we do not make use of the direct images taken with the $F 814 W$ filter for 3D-HST, but rather use the deeper, publicly available CANDELS mosaics in this band. The majority of the 3D-HST data were obtained between 2010 October and 2012 May, with two pointings in the AEGIS field obtained in 2012 December. This paper makes use of all 124 pointings, as well as 28 pointings in the GOODS-N field, which was observed between 2009 September and 2011 April in the program GO11600 (PI: B. Weiner). Each pointing was observed for two orbits, with $\sim 800$ s of direct imaging in the $F 140 \mathrm{~W}$ filter and 4511-5111 s with the G141 grism per orbit (amounting to $\sim 0.3$ orbits of imaging data per pointing in total). The average $5 \sigma$ depth of the $F 140 \mathrm{~W}$ images is $J H_{F 140 W} \sim 25.8$ mag within a $1^{\prime \prime}$ aperture. The point-source depth is 0.05 mag brighter than this, after correcting the depth for the flux outside of the $1^{\prime \prime}$ aperture using the growth curve; see Section 3.3.

The HST/WFC3 observations for the CANDELS survey cover all five fields and have a two-tiered depth structure. The "wide" observations cover $\sim 800 \operatorname{arcmin}^{2}$ combined over the five fields to $2 / 3$-orbit depth in $F 125 \mathrm{~W}$ and 4/3-orbit depth in $F 160 \mathrm{~W}$. The $F 160 \mathrm{~W}$ median $5 \sigma$ depths in a $1^{\prime \prime}$ aperture are 26.4, 26.4, 26.2, 26.6, 26.9, and 26.4 in the AEGIS, COSMOS, GOODS-N, GOODS-S, and UDS fields, respectively. The CANDELS "deep" observations cover a smaller $\sim 125 \operatorname{arcmin}^{2}$ area in GOODS-N and GOODS-S with four orbits in $F 125 \mathrm{~W}$ and six orbits in $F 160 \mathrm{~W}$. A third tier in depth is added by the even-deeper HUDF area in GOODS-S where the CANDELS, HUDF09 (GO: 11563; PI: G. Illingworth) and HUDF12 (GO: 12498; PI: R. Ellis) observations add up to 38 orbits in $F 125 \mathrm{~W}$, 33 orbits in $F 140 \mathrm{~W}$ and 85 orbits in $F 160 W$. The CANDELS data we use in this paper were taken between 2010 August and 2013 May 26. The final GOODS-N epoch, which was observed in 2013 August, is not included in our current mosaics. Adding this epoch was not a priority for the current release as it does not provide additional coverage but only addition depth in the "deep" portion of the field. These data will be included in future reductions. We summarize the relevant details here (for a detailed description of the CANDELS observations we refer the reader to Koekemoer et al. 2011).

AEGIS: (a.k.a. EGS) The CANDELS footprint is a rectangular region of $3 \times 15$ pointings or $\sim 6.5 \times 32.5$ (Figure 2). Observations in $F 125 \mathrm{~W}$ and $F 160 \mathrm{~W}$ were carried out in two epochs at different roll angles. The 3D-HST $F 140 \mathrm{~W}$ observations in AEGIS comprise 30 pointings. Fifteen of them are arranged in a regular $3 \times 5$ pattern covering the northwestern area of the field, minimizing overlap and maximizing coverage. Scheduling constraints limited the range of available roll angles for the remaining 15 pointings which led to gaps in the mosaic and more substantial overlap between pointings. The 3D-HST footprint in this field is smaller than the CANDELS one: approximately two-thirds of the CANDELS footprint also has 3D-HST F $140 W$ coverage.

COSMOS: The CANDELS mosaic is a rectangular region of $4 \times 11$ pointings or $\sim 8.6 \times 23.8$ (Figure 3 ). Observations in $F 125 \mathrm{~W}$ and $F 160 \mathrm{~W}$ were carried out in two epochs at the same roll angle. Deeper $F 125 W$ and $F 160 W$ observations of the TILE41 supernova (GO: 12461; PI: A. Riess) were added 


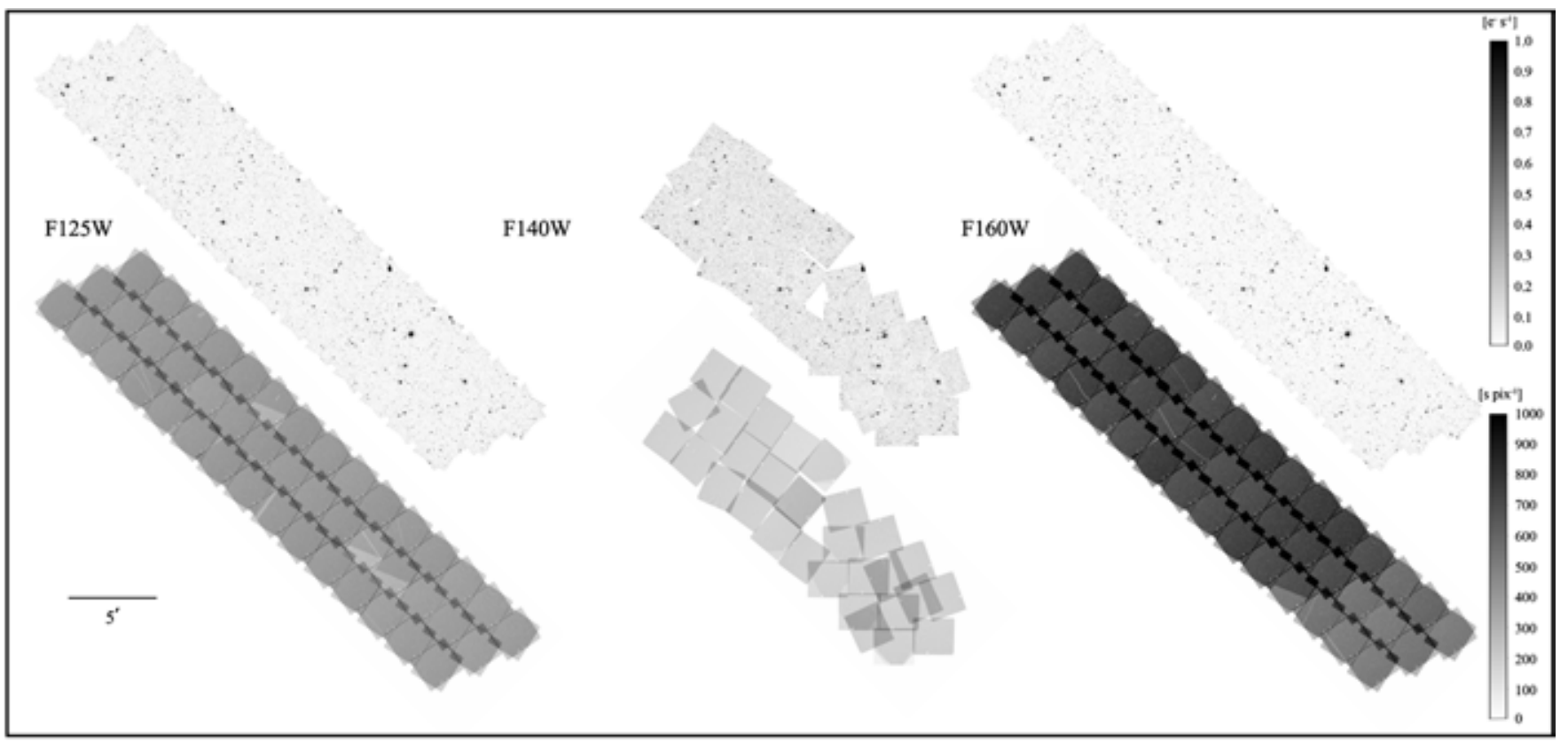

Figure 2. WFC3 mosaic science images and exposure maps for $F 125 W, F 140 W$, and $F 160 W$ in the AEGIS field. North is up, east is to the left. The science image is in units of electrons per second per pixel. The exposure map is in units of seconds per pixel. See the text for descriptions of how these mosaics were created.

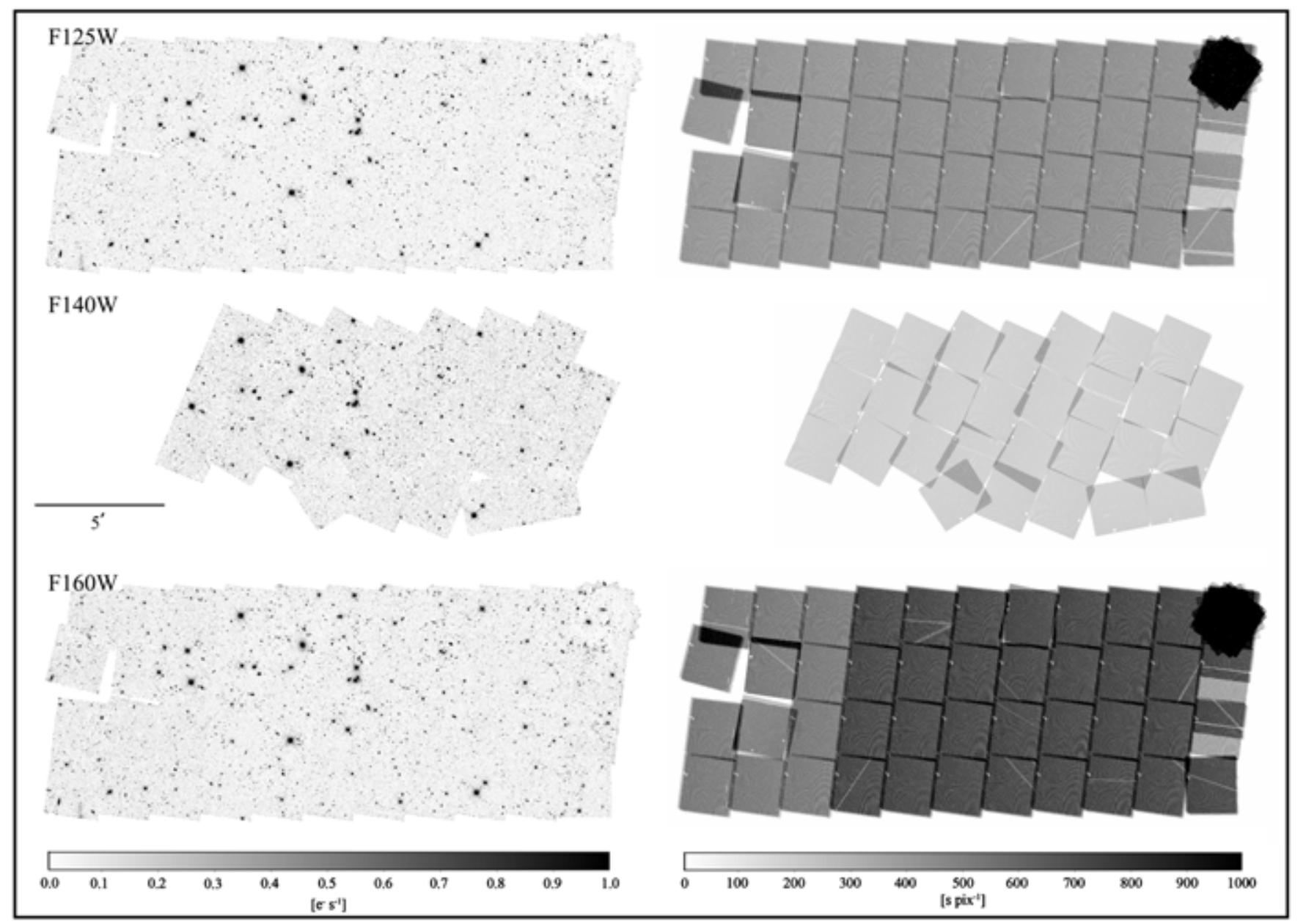

Figure 3. Same as Figure 2, for the COSMOS field. North is to the left; east is to the bottom of the page. Note that the deep region of the image saturates on the scale shown for the exposure map. 


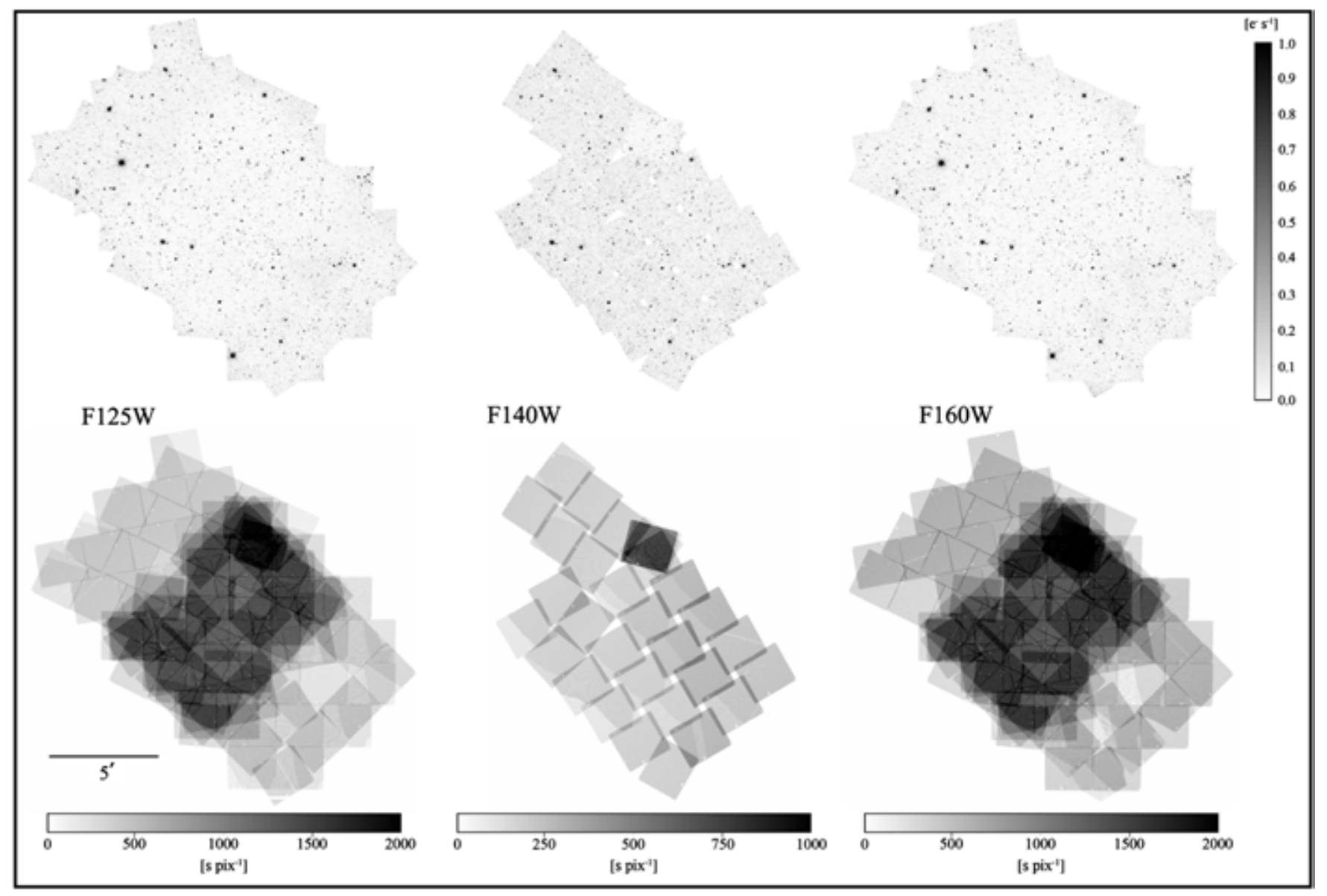

Figure 4. Same as Figure 2, for the GOODS-N field. North is up; east is to the left. Note that the scale of the $F 140 W$ weight image differs from that of $F 125 W / F 160 W$.

to our mosaics to aid in the reduction of the supernova grism observations. The 3D-HST $F 140 W$ observation in COSMOS constitute 28 pointings, most of them arranged in a $3 \times 8$ pattern. The 3D-HST footprint covers $\sim 2 / 3$ of the CANDELS footprint in this field.

GOODS-N: The CANDELS observations in this field are two-tiered. The "deep" area consists of a rectangular grid of $3 \times 5$ pointings in $F 125 W$ and $F 160 W$. The observations were done over 10 epochs (9 of which are used here) and roll angles vary by $\sim 45^{\circ}-50^{\circ}$. The remaining southern and northern areas of the field are part of the shallower tier, each covered with $\sim 2 \times 4$ pointings in both filters. The areas of the "deep" and "wide" coverage are distinctly visible in Figure 4. The $F 140 \mathrm{~W}$ observations were taken by GO: $11600^{17}$ (PI: B. Weiner) using a strategy identical to the one described above for the 3D-HST survey. The field is covered with 30 pointings arranged in a $4 \times 6$ grid with 4 additional pointings covering the northeast edge of the field. There is no $F 140 \mathrm{~W}$ imaging (or grism spectra) in the northwestern edge of the field. Additional images in $F 125 \mathrm{~W}$, $F 140 \mathrm{~W}$, and $F 160 \mathrm{~W}$ were added over the field of the COLFAX supernova (GO:12461; PI: A. Riess).

GOODS-S: The CANDELS observations in GOODS-S are also two-tiered. The "deep" area observations mirror those in GOODS-N: they cover an area of $3 \times 5$ pointings in the $F 125 W$ and $F 160 \mathrm{~W}$ filters, obtained over 10 epochs. The observations of the southern portion of the field are in the shallow tier over an area of $\sim 2 \times 4$ pointings. The northern portion of

\footnotetext{
17 http://mingus.as.arizona.edu/ bjw/aghast/
}

the field is covered by the WFC3/IR Early Release Science-2 (ERS) observations (GO/DD: 11359,11360; PI: R. O'Connell; Windhorst et al. 2011) over an area of $2 \times 4$ pointings with two orbits in each of the $F 125 \mathrm{~W}$ and $F 160 \mathrm{~W}$ filters. We have further incorporated the observations form the Hubble Ultra Deep Field 2009 (HUDF09 ${ }^{18}$ ) program (GO: 11563; PI: G. Illingworth). HUDF09 provides 34 orbits of $F 125 W$ observations and 53 orbits of $F 160 \mathrm{~W}$ observations in a single pointing in the center of the GOODS-S “deep" area. In addition, observations are carried out in two flanking fields HUDF09-1 and HUDF092 , which coincide with prior ACS coverage. The depth in HUDF09-1 is 12 orbits in $F 125 W$ and 13 orbits in $F 160 W$. The depth in HUDF09-2 is 18 and 19 orbits in the two filters, respectively. The $F 125 \mathrm{~W}$ and $F 160 \mathrm{~W}$ mosaics also include the observations of the supernova GEORGE (GO: 12099; PI: A. Riess). The 3D-HST F140W coverage in this field is broken into 38 individual pointings. Of these, 32 cover a rectangular region $\sim 8.6 \times 17.3$ in area. Two more pointings, GOODS-S1 and GOODS-S-28, cover the flanking fields. The final four pointings overlap on the HUDF area to provide deep G141 grism spectra. The $F 140 \mathrm{~W}$ mosaics also include data from the ERS2 program in a single pointing which fills a gap in the 3DHST mosaic. Finally, we added the $F 140 \mathrm{~W}$ direct observations for CDFS-AGN1 from GO:12190 (PI: A. Koekemoer), slightly extending the footprint of the mosaic. Figure 1 indicates the areas covered by different programs and Figure 5 indicates the depths across the field in each of the WFC3 bands.

\footnotetext{
18 http://archive.stsci.edu/prepds/hudf09/
} 


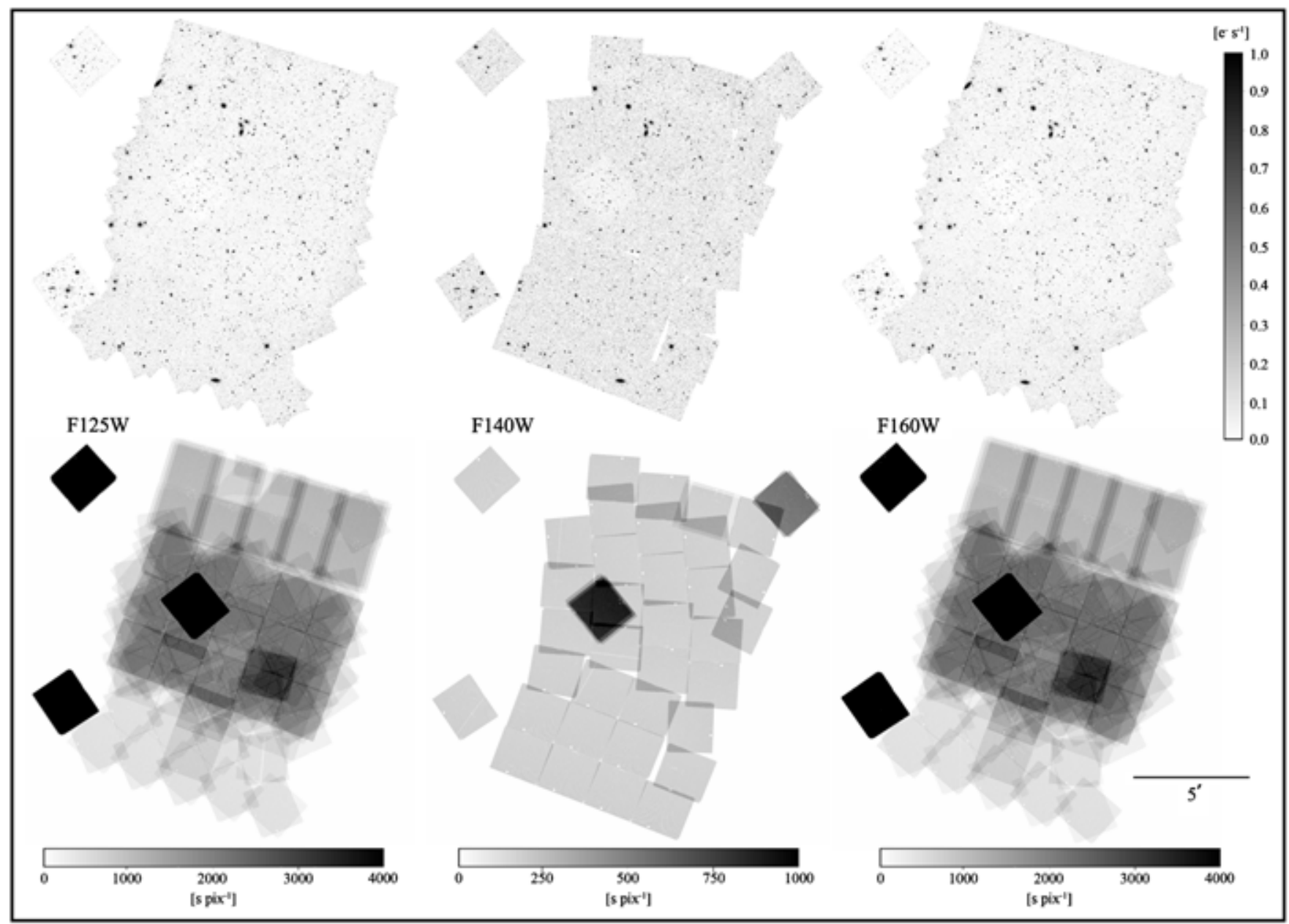

Figure 5. Same as Figure 2, for the GOODS-S field. North is up; east is to the left. Note that the scale of the $F 140 W$ weight image differs from that of $F 125 W / F 160 W$ and that the deep HUDF regions are saturated on the exposure map scale shown here.

UDS: The CANDELS mosaic is a rectangular region identical to the one in COSMOS with $4 \times 11$ pointings or $\sim 8^{\prime} .6 \times 23.8$ (Figure 6) with observations in both $F 125 W$ and $F 160 W$ taken over two epochs at the same roll angle. The pointings are arranged in a tight mosaic which maximizes contiguous coverage. Observations of the MARSHALL supernova in the $F 125 W$ and $F 160 W$ filters (GO: 12099; PI: A. Riess) are added to the mosaics. The 3D-HST $F 140 \mathrm{~W}$ observations consist of 28 pointings. Ten of the 28 pointings form a regular grid which covers the central portion of the CANDELS footprint, matching the $F 125 W / F 160 W$ roll angle. Due to scheduling constraints, the remaining 18 pointings are rotated by $\sim 45^{\circ}, 17$ of them providing a more uneven coverage of the east portion of the field. The $F 140 \mathrm{~W}$ coverage has a hole because the final pointing, UDS-18, was moved to the westernmost edge of the CANDELS coverage to carry out G141 observations of the IRC0812A $z=1.62$ cluster (Papovich et al. 2010). Figure 1 illustrates the position of UDS-18 relative to the full mosaic.

\subsubsection{Data Reduction}

We downloaded the calibrated images and association tables from the MAST archive between 2013 April and June. These images were processed on the fly with the best available calibrations at the time by the calwfc3 pipeline. We briefly summarize the calibration steps. The calwfc3 task starts with the raw files (*_raw.fits) and first populates the data quality arrays from the known bad pixel tables. It subtracts the bias for each read based on the overscan regions. It then subtracts the zeroth read to remove the bias structure across the detector, subtracts the dark current reference file based on the readout sequence, and performs a nonlinearity correction. Following these corrections on the individual reads, the task does an upthe-ramp fit to each pixel to maximize the dynamic range of the images and identify cosmic rays. The count rate is computed from the unflagged reads for each pixel and stored in the final calibrated exposure. The uncertainty in the slope is stored as the error array. Finally, the appropriate multiplicative corrections for the gain and the flat-field are applied. The resulting images (*_ft.fits) are placed on the STAGE drive and downloaded via FTP. The flat-field correction is reapplied as described below.

A number of corrections are applied to improve the data quality and produce the final data products: masking satellite trails, persistence correction, sky-subtraction, flat-field re-application, initial astrometric alignment, and additional cosmic-ray and bad pixel rejection. Some of the these steps have already been described in Brammer et al. (2012b) in the context of the $F 140 \mathrm{~W}$ and G141 reduction. These are briefly summarized here with more attention given to new steps.

The pipeline-reduced images occasionally contain satellite trails and other cosmetic blemishes, which we identify by visually inspecting all *_ft.fits images. When necessary we create mask files which mark the positions of any cosmetic blemishes in the following manner. Each $*$ ftt.fits image is displayed in DS9 and blemishes are marked with a polygon 

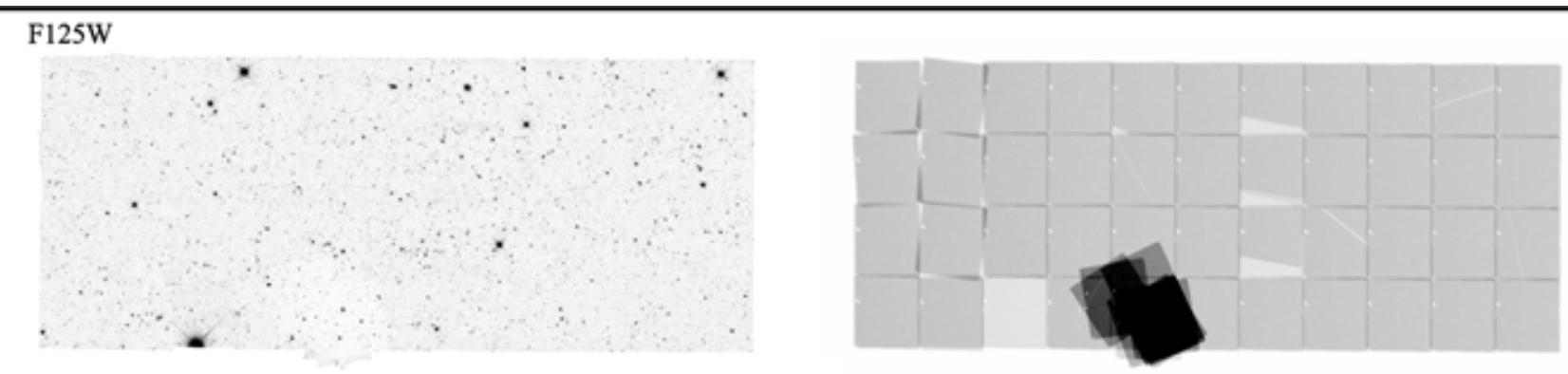

F140W
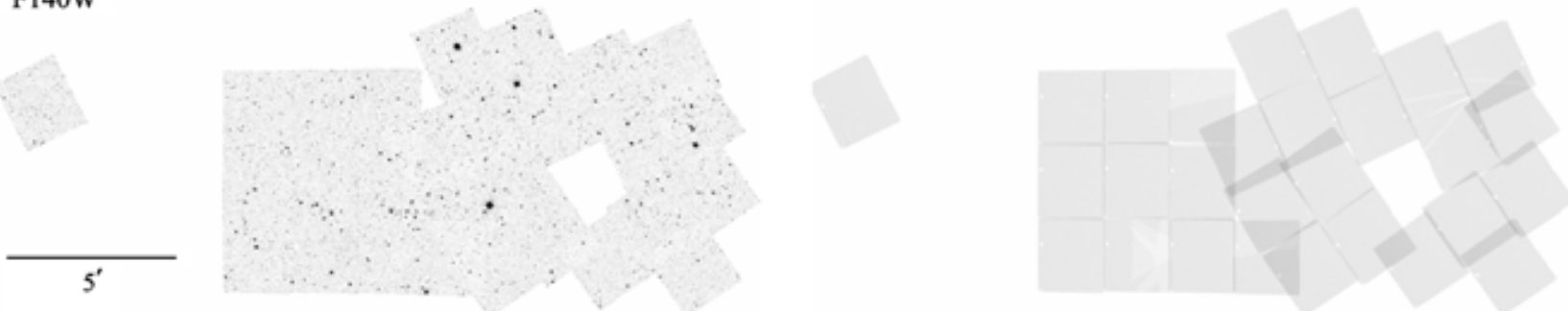

F160W
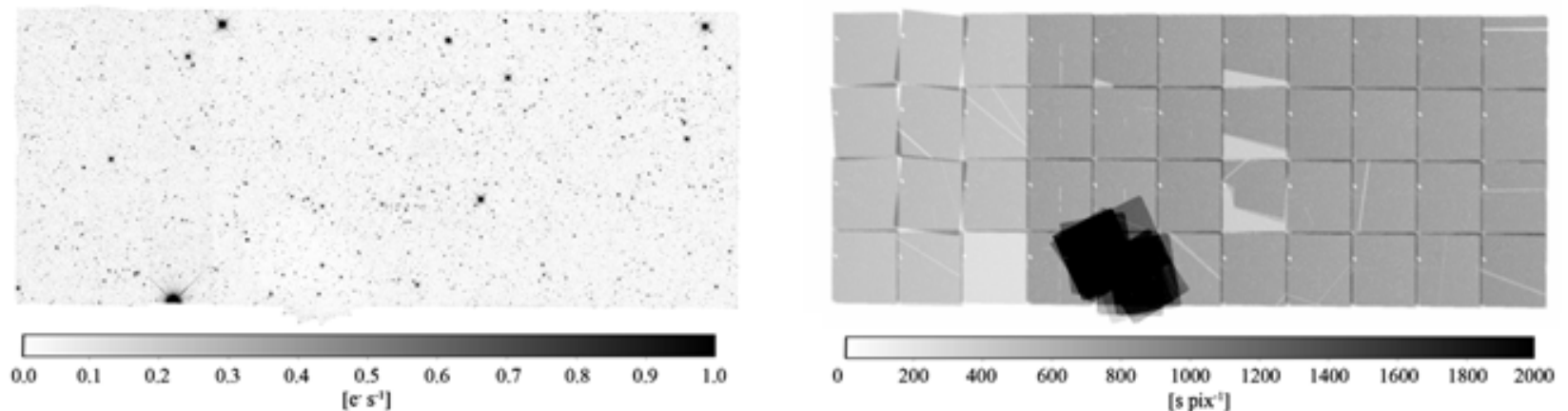

Figure 6. Same as Figure 2 for the UDS field. North is up; east is to the left. Note that the deep region of the image saturates on the scale shown for the exposure map.
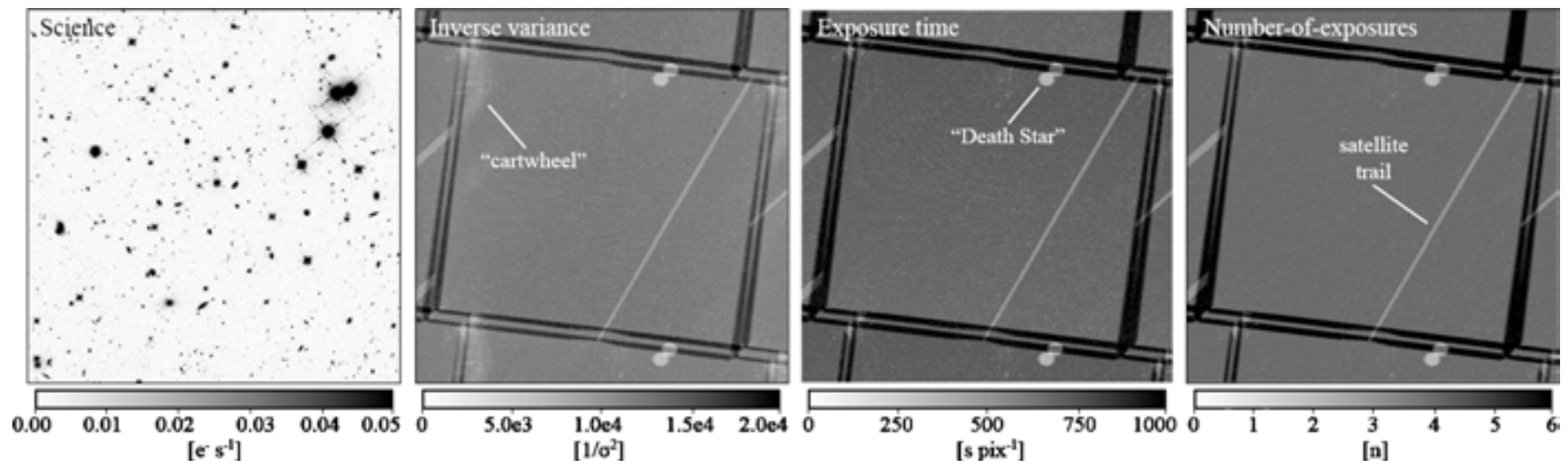

Figure 7. WFC3 science images are accompanied by three types of weight maps: inverse variance, exposure time, and number-of-exposures. We show an area of the COSMOS $F 160 \mathrm{~W}$ mosaic (pointing COSMOS-V24/COSMOS-V68) to illustrate the differences in the weight maps. Each cutout is $\sim 2.5$ on the side. A scale for each of the images is provided below the cutout. All three weight maps reflect masked and flagged pixels (satellite trails, the "Death Star"), but only the inverse variance map takes into account other error sources (flat field, background subtraction, read noise, dark current, etc.). All weight maps, as well as area maps that can be used for clustering analysis, are made available as part of the data release.

region. The coordinates of all regions are saved and then used in the reduction to set the 2048 bit for the corresponding pixels in the quality array (i.e., interpreted and ignored as a cosmic ray). The positions of such masked blemishes can be seen in the weight images; an example is shown in Figure 7. Contamination from scattered light from the bright Earth limb has been noticed in some $F 140 \mathrm{~W}$ images, especially in the GOODS-N field. Occasionally, a similar issue affects $F 125 \mathrm{~W}$ and $F 160 W$ exposures. For the current release we mitigate the problem by masking the affected exposures in the same manner as the satellite trails. In future releases, the $F 125 \mathrm{~W}$ and $F 160 \mathrm{~W}$ issue will be treated more carefully by removing only the affected reads (typically, at the beginning or the end of the exposure). Unfortunately, the short direct $F 140 \mathrm{~W}$ observations will not benefit from the new approach as they are comprised of very few reads.

Persistence is a concern with the WFC3/IR detector, causing residual flux in an exposure from bright targets in preceding separate observing programs or in other exposures within the same program. The 3D-HST observations interleave the direct 
$F 140 W$ images and the G141 grism spectra and persistence from the spectra of bright stars commonly occurs in the subsequent direct images. The spatial extent of the persistent flux is larger than the dither pattern steps and they are not usually flagged by Multidrizzle. The imaging observations from the other grism programs incorporated in our mosaics suffer the same effect. We download the persistence images provided by STScI (*_persist.fits), which provide estimates for the total persistence, for all grism programs as well as the HUDF09 program from the MAST archive. ${ }^{19}$ Rather than subtract the persistence, we adopt a more conservative approach and use the persistence images to flag affected pixels. For each individual image, we create a mask of the image where the persistence flux is greater than 0.6 times the error, convolve the mask with a maximum filter to grow the area slightly and then set the masked pixels in the error array to 4196 . These are later treated as cosmic rays and are not used in the final mosaics. The most severe persistence in the CANDELS observations was masked by hand as described above for the satellite trails.

Even though HST is above Earth's atmosphere the nearIR background is non-negligible, with the background arising predominantly from zodiacal light. The background subtraction of the $F 140 W$ images is described in detail by Brammer et al. (2012b). For each association table, we align the images to each other using tweakshifts and then create a combined pointing image with Multidrizzle. Objects are detected in the combined image using SExtractor and masked aggressively in the original distorted frames. The background is determined in two passes: first a median is subtracted, then a first-order polynomial is fit to the background and subtracted. The same procedure is applied to the $F 125 W$ and $F 160 W$ images.

We find that the standard flat applied as part go the calwfc3 task is insufficient to correct for the time-variable behavior of some features, namely the appearance of new "IR blobs" with time. We therefore create and apply time-dependent flats in the three WFC3/IR filters by splitting the CANDELS and 3D-HST observations in epochs (two for $F 125 \mathrm{~W}$ and $F 160 \mathrm{~W}$, three for $F 140 \mathrm{~W}$ ) and creating super-sky flats from the masked science exposures themselves with a method similar to that described by Pirzkal et al. (2011).

We use the Multidrizzle software (Koekemoer et al. 2003) to identify hot pixels and cosmic rays not flagged by the instrument calibration pipeline (Brammer et al. 2012b). This step is applied separately for each epoch of the CANDELS observations to avoid flagging the diffraction spikes of stars (which vary between epochs due to the different rotation angles). In the "wide" areas of CANDELS where each epoch only has two exposures, some cosmic rays and hot pixels may be missed. Therefore, our mosaics may appear less cosmetically clean. We accept this compromise to preserve the structure of stellar PSFs, as otherwise the core of the PSF and the diffraction spikes are frequently flagged as cosmic rays. We adopt a conservative value of the relevant Multidrizzle parameter, DRIZ_CR_SCALE = "2.5 0.7".

In order to provide sub-pixel sampling and mitigate the effects of hot pixels and other artifacts, all observations are dithered between exposures. Both CANDELS and 3D-HST observations employ a four-point dither pattern that provides half-pixel subsampling. In 3D-HST, all four exposures are taken during a single visit (see Brammer et al. 2012b), while for CANDELS-Wide, two exposures are taken during each of two

\footnotetext{
19 http://archive.stsci.edu/prepds/persist/
}

epochs (Koekemoer et al. 2011). The largest blemish on the WFC3/IR detector is a circle of dead pixels $\sim 50$ pixels in diameter, dubbed the "Death Star." The CANDELS epoch-toepoch dither steps are large enough to cover the hole; however, $3 \mathrm{D}$-HST only has a single epoch with small $\leqslant 10$ pixels offsets. Therefore, the "Death Star" is present in the $F 140 W$ mosaics.

At the end of the processing steps, we add back a constant background estimated by comparing the median levels in the pipeline and the processed FLT images. This is done so that the Poisson term from the background subtraction is added to the pixel errors by Astrodrizzle when creating the final mosaics.

\subsubsection{The WFC3/IR Mosaics}

Finally, the individual processed images are corrected for distortions and combined into mosaics for each field and each filter. A total of 3477 individual * flt.fits images containing $3.77 \times 10^{9}$ pixels are combined to produce these mosaics. Here we describe the alignment to a common world coordinate system (WCS) and the production of the final mosaics.

Due to uncertainties of the guide star positions, small adjustments to the commanded telescope positions are needed to align the individual images with accuracy $<1$ pixel. We use Tweakreg (version 1.2.1, updated 2013 January 25) to align each image to an external reference frame. Tweakreg fits for small differences in position, orientation, and scale between adjacent HST pointings. Measurements of positions of objects are used to determine the relative offsets; the WCS information in the image header is used as an initial estimate. The source finding algorithm used by Tweakreg has been optimized for point sources, which are sparse in the deep extragalactic fields of 3D-HST/CANDELS. For this reason we do not rely on the automatic source finding algorithm, but supply a reference catalog to be used in place of a catalog extracted from the reference image. This procedure allows us to use all the sources in the image for the alignment and enables a transformation to an absolute reference frame. The reference catalogs are created using SExtractor v2.8.6 (Bertin \& Arnouts 1996) on the following publicly available images: AEGIS, ACS- $F 814 W$ (Davis et al. 2007); COSMOS, WFC3-F160W (v1.0, Koekemoer et al. 2011); GOODS-N and GOODS-S, ACS-F814W; and UDS, WFC3-F160W (v1.0; Koekemoer et al. 2011). The 3D-HST $F 140 \mathrm{~W}$ images are shallower than the CANDELS $F 125 \mathrm{~W}$ and $F 160 \mathrm{~W}$ images. For these images, we used a magnitude limited catalog $(F 814 W, F 160 W<24)$ to decrease the rms of the differences in matched positions. For the UDF parallel fields in GOODS-S, HUDF09-01, and HUDF09-02, we used the $F 850 L P$ tiles 42 and 25 respectively from the GEMS survey (Rix et al. 2004). Aside from the reference catalog input variables, we used the defaults for all other Tweakreg parameters. The typical rms in the differences of matched positions is 0.4 native pixels, corresponding to an uncertainty of $\approx 0.3$ pixels or $\approx 0$ '.03 in the positions of the (generally faint, spatially extended) objects that were used in the procedure.

The final mosaics in each filter are produced with AstroDrizzle (version 1.1.9.dev23803, 2013 February 6). We use inversevariance weighting, a square kernel, and pixfrac $=0.8$. In order to exactly match the pixel scale $(0.06)$, the center, and the tangent point of our output mosaics to those produced by CANDELS we provide AstroDrizzle with reference images from the CANDELS public data releases (v0.5 for AEGIS and GOODS-N; v1.0 for COSMOS, GOODS-S, and UDS). The publicly available GOODS-S v1.0 images from CANDELS cover an area much larger than the WFC3 data which makes 
the final file sizes unwieldy. Our final mosaics are matched to a cropped version of the CANDELS mosaic such that the $x$ and $y$ positions of all objects are smaller by 5000 and 11,000 pixels, respectively. We create an association table of all images taken with a given filter and use it as an input to AstroDrizzle. The final images have a pixel scale of 0.06 pixel $^{-1}$. A small portion of the UDS-18 F140W 3D-HST pointing falls outside the CANDELS footprint, however all other pointings are fully contained within the mosaics. The reduction process is very similar to that used to produce the publicly available CANDELS mosaics, as described in Koekemoer et al. (2011). A key difference is that we include all the available epochs, as well as observations from other programs. Other differences include the lower threshold for cosmic ray rejection, the application of a persistence correction, the time-variable post-pipeline flat-fielding, the use of different reference images for astrometric alignment and Astrodrizzle rather than MultiDrizzle for the production of the final mosaics.

Accompanying the images are exposure maps, inverse variance weight maps and number-of-exposures maps. These outputs are used throughout the photometric analysis described below to calculate the errors, determine the depths and define source reliability flags. The inverse variance and exposure maps are standard outputs of AstroDrizzle and were created by doing consecutive runs with different final_wht_type selection. The exposure map is in units of seconds. In order to preserve flux, the exposure time in each original pixel is divided by the ratio in the areas of the original to the final pixels. The inverse variance weight map is based on the flat-field reference file and computed dark value from the image header and the final weight image accounts for all background noise sources (sky level, read noise, dark current, etc.) but not the Poisson noise from the objects themselves. The inverse variance weight map is used as a weight image input for SExtractor. The number-ofexposures map encodes the number of individual images that have contributed to the flux in each pixel. These are produced from the context images output by AstroDrizzle. A comparison of the three weight maps for a region of the COSMOS field is shown in Figure 7. The maps have different scales but generally reflect the same features because flagged pixels are taken into account by all of them. Features in the flat field (the "cartwheel" and the IR-blobs which are too small to be visible in the figure) are only visible in the inverse variance map. The moire patterns in the exposure and inverse variance maps are real and result from the dithering.

The WFC3 mosaics and all weight images are made available as part of the data release.

\subsection{Additional Data}

We use publicly available ancillary data from many different sources to build a comprehensive photometric catalog for each field. The image sources are listed in Table 3 and described in the section for each field below. Figure 8 shows the set of filter curves used for each field, together with examples of the resulting SEDs. The wavelength coverage in each field is excellent, spanning from the $U$ band through $8 \mu \mathrm{m}$. Each of the images was matched to the same pixel grid as the WFC3 images using the IRAF task wregister. The GOODS-N and UDS IRAC $8 \mu \mathrm{m}$ images showed a clear shift with respect to the WFC3 images. In these cases the image registration was improved by using the iraf task xregister, which crosscorrelates source positions to determine the shifts between the images.
We obtained all HST ACS images from the CANDELS program, and as we used the CANDELS coordinate system for our WFC3 mosaics these data are exactly aligned with ours. However, the ground-based and Spitzer IRAC data exhibit small, position-dependent astrometric offsets, which are due to a combination of the use of slightly different absolute reference systems and small position-dependent errors in the astrometry of the various data sets. The photometry software we use, described in Section 3.5, fits not only for the position dependence of the PSF but also for position-dependent astrometric errors. Information on astrometric offsets will be supplied with the release of the ancillary data.

\subsubsection{AEGIS}

The 3D-HST AEGIS field lies within the larger Extended Groth Strip (EGS), which has publicly available imaging at many wavelengths from the All-wavelength Extended Groth Strip International Survey (AEGIS; Davis et al. 2007). We incorporate imaging in 23 filters into the AEGIS photometric catalog. In the optical, we use ugriz Deep Canada-France-Hawaii Telescope Legacy Survey (CFHTLS) broadband images from the CARS team (Erben et al. 2009; Hildebrandt et al. 2009) and our own reduction of the $H S T$ /ACS $F 606 W$ and $F 814 W$ images from the CANDELS survey. In the NIR, we use $J H K_{S}$ imaging from the WIRCam Deep Survey (WIRDS; McCracken et al. 2010; Bielby et al. 2012) and $J 1, J 2, J 3, H 1, H 2, K$ from the NEWFIRM Medium-Band Survey (NMBS; Whitaker et al. 2011). The IRAC 3.6, $4.5 \mu \mathrm{m}$ images are from the Spitzer Extended Deep Survey (SEDS; Ashby et al. 2013) v1.2 data release, while the 5.8 and $8 \mu \mathrm{m}$ images are from the EGS (Barmby et al. 2008). The central wavelength of the filter, $95 \%$ cumulative throughput width of the filter, dust attenuation from Galactic extinction, aperture used for photometry, image zero point, average FWHM, and median $5 \sigma$ depth for each of the images are listed in Table 4. The depths are calculated as the median of five times the total error in each band for all the objects in the catalog, in magnitudes.

\subsubsection{COSMOS}

The 3D-HST COSMOS field is within the Cosmic Evolution Survey field (Scoville et al. 2007) and has a wealth of publicly available ancillary data. The catalog includes a total of 44 bands -27 broad bands and 17 medium bands that span both the optical and NIR. We use ugriz broadband images from the final release of the Deep Canada-France-Hawaii Telescope Legacy Survey (CFHTLS; Cuillandre et al. 2012), deep Subaru broadband images in the $B, V, r^{\prime}, i^{\prime}$, and $z^{\prime}$ bands $^{20}$ and 12 Subaru optical medium bands (Taniguchi et al. 2007). We add the CANDELS ACS/ F606W and ACS/ F814W bands using the publicly released v1.0 mosaics. ${ }^{21}$ In the NIR we use images from the UltraVISTA survey in $Y, J, H$, and $K_{s}$ (McCracken et al. 2012), $J 1, J 2, J 3, H 1, H 2, K$ from NMBS (Whitaker et al. 2011) and $J H K_{S}$ from WIRDS (McCracken et al. 2010; Bielby et al. 2012). The IRAC 3.6, $4.5 \mu \mathrm{m}$ images are from the SEDS v1.2 data release (Ashby et al. 2013), while the 5.8 and $8 \mu \mathrm{m}$ images are from the S-COSMOS survey (Sanders et al. 2007). The central wavelength of the filter, $95 \%$ cumulative throughput width of the filter, dust attenuation from Galactic extinction,

\footnotetext{
20 Note that these bands are designated $B_{J}, V_{J}, r^{+}, i^{+}$and $z^{+}$in some previous works on the COSMOS field (e.g., Whitaker et al. 2011). We use the Subaru naming convention here for consistency between the fields.

21 http://candels.ucolick.org/data_access/COSMOS.html
} 
Table 3

Image Sources

\begin{tabular}{|c|c|c|c|c|}
\hline Field & Filters & Telescope/Instrument & Survey & Reference \\
\hline \multirow[t]{8}{*}{ AEGIS } & $u, g, r, i, z$ & CFHT/MegaCam & CFHTLS & Erben et al. (2009), Hildebrandt et al. (2009) \\
\hline & $F 606 W, F 814 W$ & $H S T / \mathrm{ACS}$ & CANDELS & Grogin et al. (2011), Koekemoer et al. (2011) \\
\hline & $J 1, J 2, J 3, H 1, H 2, K$ & KPNO 4m/NEWFIRM & NMBS & Whitaker et al. (2011) \\
\hline & $J, H, K_{s}$ & CFHT/WIRCam & WIRDS & Bielby et al. (2012) \\
\hline & $F 140 W$ & $H S T / \mathrm{WFC} 3$ & 3D-HST & Brammer et al. (2012b) \\
\hline & $F 125 W, F 160 W$ & HST/WFC3 & CANDELS & Grogin et al. (2011), Koekemoer et al. (2011) \\
\hline & $3.6,4.5 \mu \mathrm{m}$ & Spitzer/IRAC & SEDS & Ashby et al. (2013) \\
\hline & $5.8,8 \mu \mathrm{m}$ & Spitzer/IRAC & EGS & Barmby et al. (2008) \\
\hline \multirow[t]{10}{*}{ COSMOS } & $u, g, r, i, z$ & CFHT/MegaCam & CFHTLS & Erben et al. (2009), Hildebrandt et al. (2009) \\
\hline & $B, V, r^{\prime}, i^{\prime}, z^{\prime}, 12$ medium-band optical & Subaru/Suprime-Cam & & Taniguchi et al. (2007) \\
\hline & $F 606 W, F 814 W$ & $H S T / \mathrm{ACS}$ & CANDELS & Grogin et al. (2011), Koekemoer et al. (2011) \\
\hline & $J 1, J 2, J 3, H 1, H 2, K$ & KPNO 4 m/NEWFIRM & NMBS & Whitaker et al. (2011) \\
\hline & $J, H, K_{s}$ & CFHT/WIRCam & WIRDS & Bielby et al. (2012) \\
\hline & $Y, J, H, K_{s}$ & VISTA & UltraVISTA & McCracken et al. (2012) \\
\hline & $F 140 W$ & $H S T / \mathrm{WFC} 3$ & 3D-HST & Brammer et al. (2012b) \\
\hline & $F 125 W, F 160 W$ & $H S T / W F C 3$ & CANDELS & Grogin et al. (2011), Koekemoer et al. (2011) \\
\hline & $3.6,4.5 \mu \mathrm{m}$ & Spitzer/IRAC & SEDS & Ashby et al. (2013) \\
\hline & $5.8,8 \mu \mathrm{m}$ & Spitzer/IRAC & S-COSMOS & Sanders et al. (2007) \\
\hline \multirow[t]{9}{*}{ GOODS-N } & $U$ & KPNO 4 m/Mosaic & Hawaii HDFN & Capak et al. (2004) \\
\hline & $G, R_{s}$ & Keck/LRIS & & Steidel et al. (2003) \\
\hline & $F 435 W, F 606 W, F 775 W, F 850 L P$ & $H S T / \mathrm{ACS}$ & GOODS & Giavalisco et al. (2004) \\
\hline & $B, V, R_{c}, I_{c}, z^{\prime}$ & Subaru/Suprime-Cam & Hawaii HDFN & Capak et al. (2004) \\
\hline & $F 140 W$ & $H S T / \mathrm{WFC} 3$ & 3D-HST & Brammer et al. (2012b) \\
\hline & $F 125 W, F 160 W$ & HST/WFC3 & CANDELS & Grogin et al. (2011), Koekemoer et al. (2011) \\
\hline & $J, H, K_{s}$ & Subaru/MOIRCS & MODS & Kajisawa et al. (2011) \\
\hline & $3.6,4.5 \mu \mathrm{m}$ & Spitzer/IRAC & SEDS & Ashby et al. (2013) \\
\hline & $5.8,8 \mu \mathrm{m}$ & Spitzer/IRAC & GOODS & Dickinson et al. (2003) \\
\hline \multirow[t]{11}{*}{ GOODS-S } & $U, R$ & VLT/VIMOS & $\mathrm{ESO} / \mathrm{GOODS}$ & Nonino et al. (2009) \\
\hline & $U 38, B, V, R_{c}, I$ & WFI $2.2 \mathrm{~m}$ & GaBoDs & Hildebrandt et al. (2006), Erben et al. (2005) \\
\hline & 14 medium bands & Subaru/Suprime-Cam & MUSYC & Cardamone et al. (2010) \\
\hline & $F 435 W, F 606 W, F 775 W, F 850 L P$ & $H S T / \mathrm{ACS}$ & GOODS & Giavalisco et al. (2004) \\
\hline & $F 606 W, F 814 W, F 850 L P$ & $H S T / \mathrm{ACS}$ & CANDELS & Grogin et al. (2011), Koekemoer et al. (2011) \\
\hline & $F 140 W$ & $H S T / \mathrm{WFC} 3$ & 3D-HST & Brammer et al. (2012b) \\
\hline & $F 125 W, F 160 W$ & $H S T / \mathrm{WFC} 3$ & CANDELS & Grogin et al. (2011), Koekemoer et al. (2011) \\
\hline & $J, H, K_{s}$ & VLT/ISAAC & ESO/GOODS, FIREWORKS & Retzlaff et al. (2010), Wuyts et al. (2008) \\
\hline & $J, K_{s}$ & CFHT/WIRcam & TENIS & Hsieh et al. (2012) \\
\hline & $3.6,4.5 \mu \mathrm{m}$ & Spitzer/IRAC & SEDS & Ashby et al. (2013) \\
\hline & $5.8,8 \mu \mathrm{m}$ & Spitzer/IRAC & GOODS & Dickinson et al. (2003) \\
\hline \multirow[t]{8}{*}{ UDS } & $U$ & CFHT/MegaCam & & O. Almaini et al./S. Foucaud et al. in preparation \\
\hline & $B, V, R_{c}, i^{\prime}, z^{\prime}$ & Subaru/Suprime-Cam & SXDS & Furusawa et al. (2008) \\
\hline & $F 606 W, F 814 W$ & $H S T / \mathrm{ACS}$ & CANDELS & Grogin et al. (2011), Koekemoer et al. (2011) \\
\hline & $F 140 W$ & HST/WFC3 & 3D-HST & Brammer et al. (2012b) \\
\hline & $F 125 W, F 160 W$ & $H S T / \mathrm{WFC} 3$ & CANDELS & Grogin et al. (2011), Koekemoer et al. (2011) \\
\hline & $J, H, K_{s}$ & UKIRT/WFCAM & UKIDSS DR8 & O. Almaini et al. in preparation \\
\hline & $3.6,4.5 \mu \mathrm{m}$ & Spitzer/IRAC & SEDS & Ashby et al. (2013) \\
\hline & $5.8,8 \mu \mathrm{m}$ & Spitzer/IRAC & SpUDS & J. Dunlop et al. in preparation \\
\hline
\end{tabular}

aperture used for photometry, image zero point, average FWHM, and median $5 \sigma$ depth for each of the images are listed in Table 5 .

The centers of bright stars are masked in the five optical broadband and 12 medium-band Subaru images, which results in artifacts in these localized regions after registration to the finer WFC/HST pixel scale. To avoid these artifacts, we flag the centers of these bright stars and grow the area by a factor of ten $\left(0{ }^{\prime} 6\right)$. Although no weight maps were publicly released for these data sets, we generate our own maps that mark the pixels flagged during this process.

\subsubsection{GOODS-North}

The GOODS-North field is a large field with GOODS ACS imaging centered on the Hubble Deep Field North (Dickinson et al. 2003). We use images in 22 filters for the GOODS-North catalog, many of which are provided by the GOODS team. The ACS $F 435 W, F 606 W, F 775 W$, and $F 850 L P$ mosaics are from the v2.0 data release (HST Cycle 11, program IDs 9425 and 9583; Giavalisco et al. 2004). The $U$-band image was taken with the Mosaic camera on the Kitt Peak 4-m telescope by the Hawaii Hubble Deep Field North project (Capak et al. 2004). ${ }^{22}$ Broadband optical data in the $B V R i z$ filters from Suprime-Cam on the Subaru $8.2 \mathrm{~m}$ are also provided by the Hawaii Hubble Deep Field North project. Optical $G$ and $R_{s}$ band images from LRIS on the Keck I telescope are provided by Steidel et al. (2004), Reddy et al. (2005). We use the HST/

\footnotetext{
22 http://www.astro.caltech.edu/ capak/hdf/index.html
} 


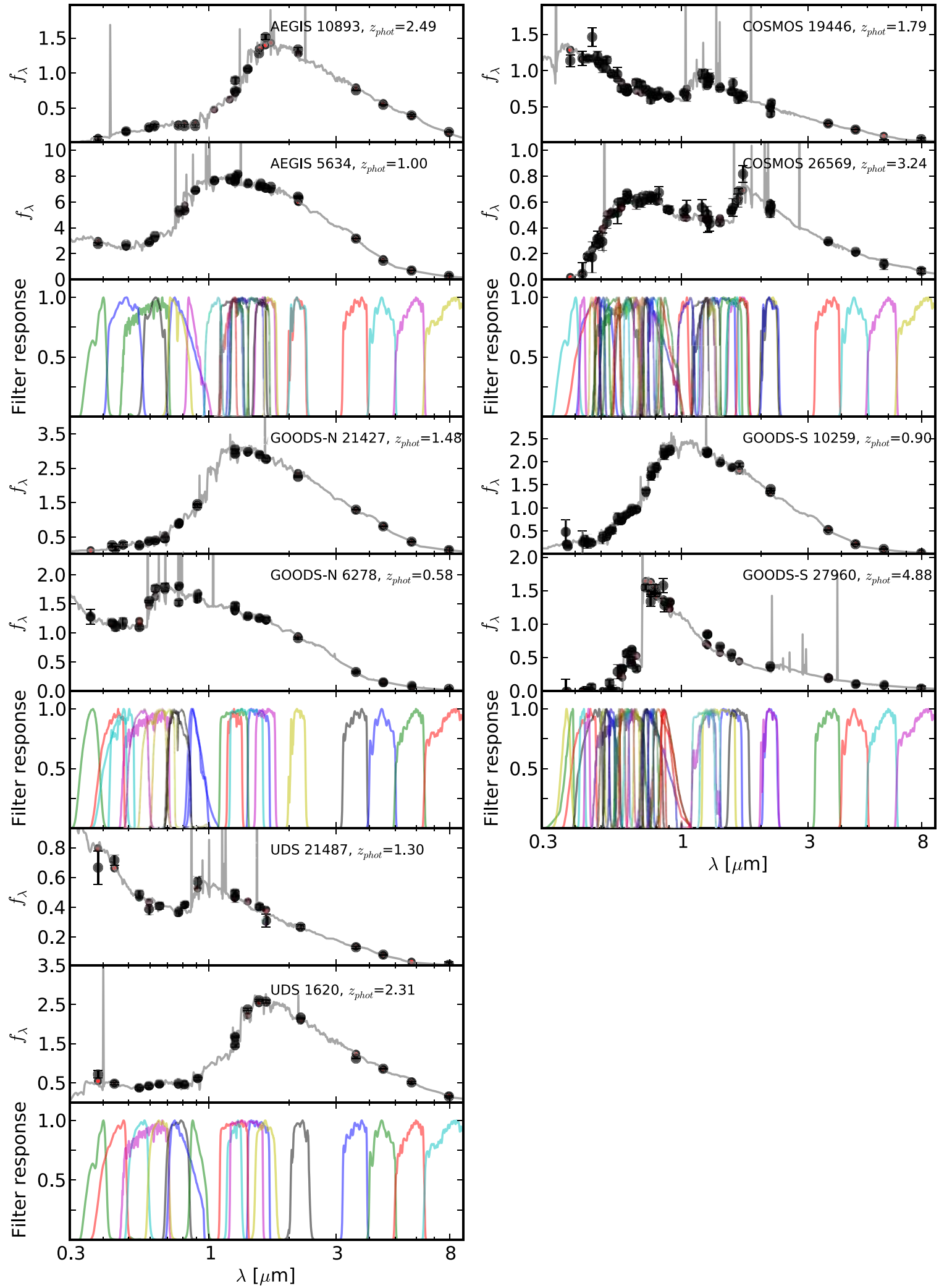

Figure 8. Photometric filter set and two example SEDs for each of the five fields. The lower panels show the full set of filter curves used for the photometric catalog of each field, normalized to a maximum transmission of one. The upper two panels show the SEDs of two galaxies randomly chosen to demonstrate some of the variety of objects in the catalogs. The id and redshift of each object are shown in the top right corner. The black circles indicate the observed flux in each filter, the best-fit EAZY template spectra are shown in gray with the expected flux from the fit for each band in that catalog shown by the red circles. Some objects lie in areas of the image without coverage in one or more bands; in these cases only the predicted flux (red point) is shown. COSMOS and the GOODS-South field are particularly well sampled by the medium bands in the optical region, but there is excellent multi-wavelength coverage in all of the fields.

(A color version of this figure is available in the online journal.) 
Table 4

AEGIS Imaging Data

\begin{tabular}{lccccccc}
\hline \hline Band & $\begin{array}{c}\lambda_{\text {central }} \\
(\mu \mathrm{m})\end{array}$ & $\begin{array}{c}\text { Width }^{\mathrm{a}} \\
(\mu \mathrm{m})\end{array}$ & $\begin{array}{c}A_{\lambda} \\
(\mathrm{mag})\end{array}$ & $\begin{array}{c}\text { Aperture } \\
(\operatorname{arcsec})\end{array}$ & $\begin{array}{c}\text { Zero Point } \\
(\mathrm{AB})\end{array}$ & $\begin{array}{c}\text { FWHM } \\
(\operatorname{arcsec})\end{array}$ & $\begin{array}{c}5 \sigma \text { Depth }^{\mathrm{b}} \\
(\mathrm{mag})\end{array}$ \\
\hline$U$ & 0.3828 & 0.0771 & 0.037 & 1.2 & 30.000 & 0.88 & 26.8 \\
$G$ & 0.4870 & 0.1428 & 0.030 & 1.2 & 30.000 & 0.81 & 27.4 \\
$F 606 W$ & 0.5921 & 0.2225 & 0.023 & 0.7 & 26.491 & 0.13 & 26.8 \\
$R$ & 0.6245 & 0.1232 & 0.020 & 1.2 & 30.000 & 0.81 & 27.3 \\
$I$ & 0.7676 & 0.1501 & 0.015 & 1.2 & 30.000 & 0.81 & 27.0 \\
$F 814 W$ & 0.8057 & 0.2358 & 0.014 & 0.7 & 25.943 & 0.11 & 26.4 \\
$Z$ & 0.8872 & 0.1719 & 0.012 & 1.2 & 30.000 & 0.72 & 26.2 \\
$J 1$ & 1.0460 & 0.1471 & 0.010 & 1.5 & 23.310 & 1.13 & 24.8 \\
$J 2$ & 1.1946 & 0.1476 & 0.008 & 1.5 & 23.350 & 1.16 & 24.5 \\
$J 3$ & 1.2778 & 0.1394 & 0.006 & 1.5 & 23.370 & 1.08 & 24.4 \\
$F 125 W$ & 1.2471 & 0.2867 & 0.006 & 0.7 & 26.247 & 0.18 & 26.3 \\
$J$ & 1.2530 & 0.1541 & 0.006 & 1.2 & 30.000 & 0.76 & 24.5 \\
$F 140 W$ & 1.3924 & 0.3760 & 0.005 & 0.7 & 26.465 & 0.19 & 25.7 \\
$F 160 W$ & 1.5396 & 0.2744 & 0.004 & 0.7 & 25.956 & 0.19 & 26.1 \\
$H 1$ & 1.5601 & 0.1658 & 0.005 & 1.5 & 23.590 & 1.10 & 23.8 \\
$H 2$ & 1.7064 & 0.1721 & 0.004 & 1.5 & 23.610 & 1.06 & 23.8 \\
$H$ & 1.6294 & 0.2766 & 0.004 & 1.2 & 30.000 & 0.68 & 24.3 \\
$K$ & 2.1684 & 0.3181 & 0.003 & 1.5 & 23.850 & 1.08 & 23.6 \\
$K s$ & 2.1574 & 0.3151 & 0.003 & 1.2 & 30.000 & 0.69 & 24.0 \\
IRAC1 & 3.5569 & 0.7139 & 0.000 & 3.0 & 21.545 & 1.7 & 25.2 \\
IRAC2 & 4.5020 & 0.9706 & 0.000 & 3.0 & 21.545 & 1.7 & 25.1 \\
IRAC3 & 5.7450 & 1.3591 & 0.000 & 3.0 & 21.545 & 1.9 & 22.6 \\
IRAC4 & 7.9158 & 2.7893 & 0.000 & 3.0 & 21.545 & 2.0 & 22.6 \\
\hline & & & & & & &
\end{tabular}

Notes.

a $95 \%$ cumulative throughput width.

${ }^{\mathrm{b}}$ Median $5 \sigma$ depth calculated from the errors of objects in the final catalogs.

WFC3 $F 140 W$ images from GO: 11600 (PI: B. Weiner) and the $F 125 W$ and $F 160 W$ images from CANDELS to produce our own mosaics, as described in Section 2.1. Ground-based $J$, $H$, and $K_{s}$-band images from the Multi-Object Infrared Camera and Spectrograph (MOIRCS) on Subaru are provided by the MOIRCS Deep Survey (MODS; Kajisawa et al. 2011). ${ }^{23} \mathrm{We}$ use the "convolved" mosaics provided by the MODS team, in which the four subfields making up each mosaic have been PSFmatched to the field with the worst seeing. The IRAC 3.6, $4.5 \mu \mathrm{m}$ images are from the SEDS v1.2 data release (Ashby et al. 2013), while the 5.8 and $8 \mu \mathrm{m}$ images are from the GOODS Spitzer 2nd data release. There are two epochs of data for the 5.8 and $8 \mu \mathrm{m}$ bands. We measure fluxes independently on the two epochs and where there is overlap, combine them for the final catalog, rather than coadding the images beforehand, due to the differences in the orientation of the PSFs. The central wavelength of the filter, $95 \%$ cumulative throughput width of the filter, dust attenuation from Galactic extinction, aperture used for photometry, image zero point, average FWHM, and median $5 \sigma$ depth for each of the images are listed in Table 6.

\subsubsection{GOODS-South}

The 3D-HST GOODS-South field is centered on the Chandra Deep Field South and contains the WFC3 Early Release Science (ERS) field and the Hubble Ultra Deep Field (HUDF) within its boundaries. In the GOODS-South field we make use of images in 26 broad bands and 14 medium bands. As for GOODS-N, much of the data are publicly available from the GOODS team. Here we make use of the ACS v2.0 data as described above. In addition, we include the publicly released v1.0 CANDELS ACS $/ F 606 W$, ACS $/ F 814 W$ and ACS $/ F 850 L P$ mosaics. ${ }^{24} U$ and $R$-band images from VIMOS on the Very Large Telescope

\footnotetext{
23 http://www.astr.tohoku.ac.jp/MODS

$24 \mathrm{http} / / /$ candels.ucolick.org/data_access/GOODS-S.html
}

Table 5

COSMOS Imaging Data

\begin{tabular}{|c|c|c|c|c|c|c|c|}
\hline Band & $\begin{array}{c}\lambda_{\text {central }} \\
(\mu \mathrm{m})\end{array}$ & $\begin{array}{l}\text { Width }^{\mathrm{a}} \\
(\mu \mathrm{m})\end{array}$ & $\begin{array}{c}A_{\lambda} \\
\text { (mag) }\end{array}$ & $\begin{array}{c}\text { Aperture } \\
(\operatorname{arcsec})\end{array}$ & $\begin{array}{l}\text { Zero Point } \\
\quad(\mathrm{AB})\end{array}$ & $\begin{array}{l}\text { FWHM } \\
(\operatorname{arcsec})\end{array}$ & $\begin{array}{c}5 \sigma \text { Depth }^{\mathrm{b}} \\
(\mathrm{mag})\end{array}$ \\
\hline$U$ & 0.3828 & 0.07710 & 0.076 & 1.2 & 25.233 & 0.85 & 26.7 \\
\hline$B$ & 0.4448 & 0.1035 & 0.068 & 1.2 & 31.400 & 0.78 & 27.9 \\
\hline$G$ & 0.4870 & 0.1428 & 0.062 & 1.2 & 26.375 & 0.85 & 27.2 \\
\hline$V$ & 0.5470 & 0.0993 & 0.051 & 1.2 & 31.400 & 0.90 & 27.2 \\
\hline$F 606 \mathrm{~W}$ & 0.5921 & 0.2225 & 0.019 & 0.7 & 26.491 & 0.11 & 26.7 \\
\hline$R$ & 0.6245 & 0.1232 & 0.044 & 1.2 & 25.926 & 0.78 & 27.2 \\
\hline$R p$ & 0.6276 & 0.1379 & 0.044 & 1.2 & 31.400 & 0.84 & 27.3 \\
\hline$I$ & 0.7676 & 0.1501 & 0.032 & 1.2 & 25.703 & 0.81 & 27.0 \\
\hline$I p$ & 0.7671 & 0.1489 & 0.032 & 1.2 & 31.40 & 0.74 & 27.0 \\
\hline$F 814 W$ & 0.8057 & 0.2358 & 0.029 & 0.7 & 25.943 & 0.10 & 26.5 \\
\hline$Z$ & 0.8872 & 0.1719 & 0.025 & 1.2 & 24.768 & 0.75 & 26.0 \\
\hline$Z p$ & 0.9028 & 0.1411 & 0.025 & 1.2 & 31.400 & 0.87 & 25.8 \\
\hline UVISTA_Y & 1.0217 & 0.1026 & 0.020 & 1.2 & 30.000 & 0.79 & 25.7 \\
\hline$J 1$ & 1.0460 & 0.1471 & 0.019 & 1.5 & 23.310 & 1.19 & 24.7 \\
\hline$J 2$ & 1.1946 & 0.1476 & 0.016 & 1.5 & 23.350 & 1.17 & 24.5 \\
\hline$J 3$ & 1.2778 & 0.1394 & 0.014 & 1.5 & 23.370 & 1.12 & 24.4 \\
\hline F125W & 1.2471 & 0.2867 & 0.014 & 0.7 & 26.247 & 0.19 & 26.1 \\
\hline$J$ & 1.2530 & 0.1541 & 0.014 & 1.2 & 30.000 & 0.93 & 23.8 \\
\hline UVISTA_J & 1.2527 & 0.1703 & 0.017 & 1.2 & 30.000 & 0.78 & 25.4 \\
\hline$F 140 W$ & 1.3924 & 0.3760 & 0.012 & 0.7 & 26.465 & 0.19 & 25.5 \\
\hline$F 160 W$ & 1.5396 & 0.2744 & 0.010 & 0.7 & 25.956 & 0.19 & 25.8 \\
\hline$H 1$ & 1.5601 & 0.1658 & 0.012 & 1.5 & 23.590 & 1.03 & 23.7 \\
\hline$H 2$ & 1.7064 & 0.1721 & 0.010 & 1.5 & 23.610 & 1.24 & 23.6 \\
\hline$H$ & 1.6294 & 0.2766 & 0.009 & 1.2 & 30.000 & 0.73 & 24.0 \\
\hline UVISTA_H & 1.6433 & 0.2844 & 0.009 & 1.2 & 30.000 & 0.76 & 24.9 \\
\hline$K$ & 2.1684 & 0.3181 & 0.005 & 1.5 & 23.850 & 1.08 & 23.7 \\
\hline$K s$ & 2.1574 & 0.3151 & 0.005 & 1.2 & 30.000 & 0.68 & 23.8 \\
\hline UVISTA_Ks & 2.1503 & 0.3109 & 0.005 & 1.2 & 30.000 & 0.74 & 25.0 \\
\hline IA427 & 0.4260 & 0.0223 & 0.069 & 1.2 & 31.400 & 0.81 & 26.3 \\
\hline IA464 & 0.4633 & 0.0238 & 0.063 & 1.2 & 31.400 & 0.91 & 25.8 \\
\hline IA484 & 0.4847 & 0.0250 & 0.060 & 1.2 & 31.400 & 0.60 & 26.4 \\
\hline IA505 & 0.5061 & 0.0259 & 0.058 & 1.2 & 31.400 & 0.83 & 26.2 \\
\hline IA527 & 0.5259 & 0.0282 & 0.056 & 1.2 & 31.400 & 0.71 & 26.5 \\
\hline IA574 & 0.5763 & 0.0303 & 0.051 & 1.5 & 31.400 & 1.08 & 25.7 \\
\hline IA624 & 0.6231 & 0.0337 & 0.042 & 1.2 & 31.400 & 0.84 & 26.4 \\
\hline IA679 & 0.6782 & 0.0372 & 0.038 & 1.5 & 31.400 & 1.12 & 25.9 \\
\hline IA709 & 0.7074 & 0.0358 & 0.040 & 1.5 & 31.400 & 1.05 & 26.1 \\
\hline IA738 & 0.7359 & 0.0355 & 0.036 & 1.2 & 31.400 & 0.85 & 26.0 \\
\hline IA767 & 0.7680 & 0.0389 & 0.032 & 1.5 & 31.400 & 1.09 & 25.7 \\
\hline IA827 & 0.8247 & 0.0367 & 0.028 & 1.5 & 31.400 & 1.07 & 25.6 \\
\hline IRAC1 & 3.5569 & 0.7139 & 0.000 & 3.0 & 21.581 & 1.7 & 25.1 \\
\hline IRAC2 & 4.5020 & 0.9706 & 0.000 & 3.0 & 21.581 & 1.7 & 25.0 \\
\hline IRAC3 & 5.7450 & 1.3591 & 0.000 & 3.0 & 21.581 & 1.9 & 21.6 \\
\hline IRAC4 & 7.9158 & 2.7893 & 0.000 & 3.0 & 21.581 & 2.0 & 21.6 \\
\hline
\end{tabular}

Notes.

a $95 \%$ cumulative throughput width.

${ }^{\mathrm{b}}$ Median $5 \sigma$ depth calculated from the errors of objects in the final catalogs.

(VLT) are from the European Southern Observatory (ESO) v1.0 data release (Nonino et al. 2009). We use the ESO WFI U38, $B, V, R c$ and $I$ images reduced by the Garching-Bonn Deep Survey (GaBoDS) consortium (Hildebrandt et al. 2006; Erben et al. 2005). We incorporate 14 Subaru medium bands from the Multiwavelength Survey by Yale-Chile (MUSYC; Cardamone et al. 2010; Gawiser et al. 2006). ${ }^{25}$ Four medium bands (three of which have FWHM $>1$ 1.5) were excluded from the analysis due to large zero point uncertainties. We use our own reductions of the CANDELS data in the WFC $3 / F 125 W$ and WFC3/ $F 160 \mathrm{~W}$ bands and the 3D-HST $F 140 \mathrm{~W}$ image, as described in Section 2.1. We use the $J, H$ and $K_{s}$-bands mosaics from the FIREWORKS survey, kindly provided by S. Wuyts. The mosaics were constructed by convolving the individual ESO GOODS survey images from ISAAC on the VLT (v1.5 data

\footnotetext{
25 http://www.astro.yale.edu/MUSYC/
} 
Table 6

GOODS-N Imaging Data

\begin{tabular}{lccccccc}
\hline \hline Band & $\begin{array}{c}\lambda_{\text {central }} \\
(\mu \mathrm{m})\end{array}$ & $\begin{array}{c}\text { Width }^{\mathrm{a}} \\
(\mu \mathrm{m})\end{array}$ & $\begin{array}{c}A_{\lambda} \\
(\mathrm{mag})\end{array}$ & $\begin{array}{c}\text { Aperture } \\
(\operatorname{arcsec})\end{array}$ & $\begin{array}{c}\text { Zero Point } \\
(\mathrm{AB})\end{array}$ & $\begin{array}{c}\text { FWHM } \\
(\operatorname{arcsec})\end{array}$ & $\begin{array}{c}5 \sigma \text { Depth }^{\mathrm{b}} \\
(\mathrm{mag})\end{array}$ \\
\hline$U$ & 0.3593 & 0.0721 & 0.052 & 1.5 & 31.369 & 1.26 & 26.4 \\
$F 435 W$ & 0.4318 & 0.0993 & 0.044 & 0.7 & 25.689 & 0.10 & 27.1 \\
HDF.B & 0.4448 & 0.1035 & 0.042 & 1.0 & 31.136 & 0.71 & 26.7 \\
$G$ & 0.4751 & 0.0940 & 0.039 & 1.2 & 35.250 & 1.07 & 26.3 \\
HDF.V0201 & 0.5470 & 0.0993 & 0.033 & 1.0 & 34.707 & 0.71 & 27.0 \\
$F 606 W$ & 0.5919 & 0.2225 & 0.030 & 0.7 & 26.511 & 0.10 & 27.4 \\
HDF.R & 0.6276 & 0.1379 & 0.027 & 1.5 & 34.676 & 1.11 & 26.2 \\
$R s$ & 0.6819 & 0.1461 & 0.023 & 1.2 & 35.250 & 1.02 & 25.6 \\
HDF.I & 0.7671 & 0.1489 & 0.020 & 1.0 & 34.481 & 0.72 & 25.8 \\
$F 775 W$ & 0.7693 & 0.1491 & 0.020 & 0.7 & 25.671 & 0.11 & 26.9 \\
HDF.Z & 0.9028 & 0.1411 & 0.015 & 1.0 & 33.946 & 0.67 & 25.5 \\
$F 850 L P$ & 0.9036 & 0.2092 & 0.015 & 0.7 & 24.871 & 0.11 & 26.7 \\
$F 125 W$ & 1.2471 & 0.2867 & 0.009 & 0.7 & 26.230 & 0.18 & 26.7 \\
$J$ & 1.2517 & 0.1571 & 0.009 & 1.0 & 26.000 & 0.60 & 25.0 \\
$F 140 W$ & 1.3924 & 0.3760 & 0.007 & 0.7 & 26.452 & 0.18 & 25.9 \\
$F 160 W$ & 1.5396 & 0.2744 & 0.006 & 0.7 & 25.946 & 0.19 & 26.1 \\
$H$ & 1.6347 & 0.2686 & 0.005 & 1.0 & 26.000 & 0.60 & 24.3 \\
$K s$ & 2.1577 & 0.3044 & 0.004 & 1.0 & 26.000 & 0.60 & 24.7 \\
IRAC1 & 3.5569 & 0.7139 & 0.000 & 3.0 & 21.581 & 1.7 & 24.5 \\
IRAC2 & 4.5020 & 0.9706 & 0.000 & 3.0 & 21.581 & 1.7 & 24.6 \\
IRAC3 & 5.7450 & 1.3591 & 0.000 & 3.0 & 20.603 & 1.9 & 22.8 \\
IRAC4 & 7.9158 & 2.7893 & 0.000 & 3.0 & 21.781 & 2.0 & 22.7 \\
& & & & & & &
\end{tabular}

Notes.

a $95 \%$ cumulative throughput width.

${ }^{\mathrm{b}}$ Median $5 \sigma$ depth calculated from the errors of objects in the final catalogs.

release; Retzlaff et al. 2010) to a uniform PSF (see Wuyts et al. 2008 for details). We also include deep $J$ and $K_{s}$-band images from the Taiwan Extended Chandra Deep Field South NearInfrared Survey (TENIS; Hsieh et al. 2012). We use IRAC 3.6, $4.5 \mu \mathrm{m}$ data from SEDS v1.2 data release (Ashby et al. 2013) and 5.8 and $8 \mu \mathrm{m}$ images from the GOODS Spitzer 3rd data release. As with GOODS-N, there are two epochs of data for both the 5.8 and $8 \mu \mathrm{m}$ bands, which we treat separately, combining the two flux measurements for objects in the overlapping region for the final catalog. The central wavelength of the filter, $95 \%$ cumulative throughput width of the filter, dust attenuation from Galactic extinction, aperture used for photometry, image zero point, average FWHM, and median $5 \sigma$ depth for each of the images are listed in Table 7.

\subsubsection{UDS}

The 3D-HST UDS field covers part of the region observed by the UKIDSS UDS. The catalog incorporates photometry in 18 filters. In addition to the WFC $3 / F 140 W$ data from 3D-HST, we use our own reductions of the CANDELS WFC3/ $F 125 W$ and WFC $3 / F 160 W$ data and the ACS/ F606W and $\mathrm{ACS} / F 814 W$ data from the CANDELS v1.0 data release. ${ }^{26}$ This is supplemented by optical ground-based data from the Subaru/XMM-Newton Deep Survey (SXDS; Furusawa et al. 2008 ) in the $B, V, R, i$, and $z$-bands. ${ }^{27}$ We use the mosaics provided by M. Cirasuolo (Cirasuolo et al. 2010). ${ }^{28}$ We include a $u^{\prime}$-band image reduced from CFHT archival data by R. Williams and R. Quadri (2012, private communication). We have used the UKIDSS UDS NIR imaging data from the 8th data release. ${ }^{29}$ The UKIDSS project is defined in Lawrence et al. (2007). Further details on the UDS can be found in

\footnotetext{
26 http://candels.ucolick.org/data_access/UDS.html

$27 \mathrm{http} / / /$ www.naoj.org/Science/SubaruProject/SXDS/

28 http://www.roe.ac.uk/ ciras/Scientific_Research.html

29 http://surveys.roe.ac.uk/wsa/dr8plus_release.html
}

Table 7

GOODS-S Imaging Data

\begin{tabular}{|c|c|c|c|c|c|c|c|}
\hline Band & $\begin{array}{c}\lambda_{\text {central }} \\
(\mu \mathrm{m})\end{array}$ & $\begin{array}{l}\text { Width }^{\mathrm{a}} \\
(\mu \mathrm{m})\end{array}$ & $\begin{array}{c}A_{\lambda} \\
(\mathrm{mag})\end{array}$ & $\begin{array}{c}\text { Aperture } \\
(\operatorname{arcsec})\end{array}$ & $\begin{array}{l}\text { Zero Point } \\
\quad(\mathrm{AB})\end{array}$ & $\begin{array}{l}\text { FWHM } \\
(\operatorname{arcsec})\end{array}$ & $\begin{array}{c}5 \sigma \text { Depth }^{\mathrm{b}} \\
\text { (mag) }\end{array}$ \\
\hline U38 & 0.3637 & 0.0475 & 0.033 & 1.2 & 21.910 & 0.98 & 25.7 \\
\hline$B$ & 0.4563 & 0.0975 & 0.026 & 1.2 & 24.379 & 1.01 & 26.9 \\
\hline$V$ & 0.5396 & 0.0920 & 0.021 & 1.2 & 24.096 & 0.94 & 26.6 \\
\hline$R c$ & 0.6517 & 0.1600 & 0.016 & 1.2 & 24.651 & 0.83 & 26.6 \\
\hline$I$ & 0.7838 & 0.2459 & 0.012 & 1.2 & 23.640 & 0.96 & 24.7 \\
\hline$U$ & 0.3750 & 0.0591 & 0.032 & 1.2 & 26.150 & 0.8 & 27.9 \\
\hline$R$ & 0.6443 & 0.1333 & 0.017 & 1.2 & 27.490 & 0.7 & 27.5 \\
\hline$F 435 W$ & 0.4318 & 0.0993 & 0.028 & 0.7 & 25.690 & 0.11 & 27.3 \\
\hline$F 606 W$ cand & 0.5921 & 0.2225 & 0.019 & 0.7 & 26.493 & 0.11 & 27.2 \\
\hline$F 606 W$ & 0.5919 & 0.2225 & 0.019 & 0.7 & 26.511 & 0.11 & 27.4 \\
\hline$F 775 W$ & 0.7693 & 0.1491 & 0.013 & 0.7 & 25.671 & 0.10 & 26.9 \\
\hline$F 814 W$ & 0.8057 & 0.2358 & 0.012 & 0.7 & 25.947 & 0.10 & 27.2 \\
\hline$F 850 L P$ cand & 0.9033 & 0.2092 & 0.010 & 0.7 & 24.857 & 0.11 & 25.5 \\
\hline$F 850 L P$ & 0.9036 & 0.2092 & 0.010 & 0.7 & 24.871 & 0.11 & 26.5 \\
\hline$J$ & 1.2356 & 0.2668 & 0.005 & 1.0 & 26.000 & 0.65 & 25.1 \\
\hline TENIS J & 1.2530 & 0.1540 & 0.005 & 1.0 & 23.900 & 0.93 & 25.0 \\
\hline$F 125 W$ & 1.2471 & 0.2867 & 0.005 & 0.7 & 26.230 & 0.18 & 26.1 \\
\hline$F 140 W$ & 1.3924 & 0.3760 & 0.004 & 0.7 & 26.452 & 0.18 & 25.6 \\
\hline$F 160 W$ & 1.5396 & 0.2744 & 0.003 & 0.7 & 25.946 & 0.19 & 26.4 \\
\hline$H$ & 1.6496 & 0.2832 & 0.003 & 1.0 & 26.000 & 0.65 & 24.5 \\
\hline TENIS Ks & 2.1574 & 0.3151 & 0.002 & 1.0 & 23.900 & 0.83 & 24.5 \\
\hline$K s$ & 2.1667 & 0.2686 & 0.002 & 1.0 & 26.000 & 0.65 & 24.4 \\
\hline IA427 & 0.4260 & 0.0223 & 0.028 & 1.5 & 25.100 & 1.01 & 25.4 \\
\hline IA445 & 0.4443 & 0.0219 & 0.027 & 1.5 & 25.070 & 1.23 & 25.7 \\
\hline IA505 & 0.5061 & 0.0259 & 0.023 & 1.2 & 25.340 & 0.94 & 25.7 \\
\hline IA527 & 0.5259 & 0.0282 & 0.022 & 1.2 & 25.720 & 0.83 & 26.4 \\
\hline IA550 & 0.5495 & 0.0305 & 0.021 & 1.5 & 25.880 & 1.13 & 25.9 \\
\hline IA574 & 0.5763 & 0.0303 & 0.020 & 1.2 & 25.710 & 0.95 & 25.5 \\
\hline IA598 & 0.6007 & 0.0331 & 0.019 & 1.2 & 26.020 & 0.63 & 26.5 \\
\hline IA624 & 0.6231 & 0.0337 & 0.018 & 1.2 & 25.890 & 0.61 & 26.4 \\
\hline IA651 & 0.6498 & 0.0360 & 0.017 & 1.2 & 26.150 & 0.6 & 26.6 \\
\hline IA679 & 0.6782 & 0.0372 & 0.015 & 1.2 & 26.200 & 0.8 & 26.4 \\
\hline IA738 & 0.7359 & 0.0355 & 0.013 & 1.2 & 26.020 & 0.77 & 26.2 \\
\hline IA767 & 0.7680 & 0.0389 & 0.013 & 1.2 & 26.040 & 0.7 & 25.1 \\
\hline IA797 & 0.7966 & 0.0404 & 0.012 & 1.2 & 26.020 & 0.68 & 25.0 \\
\hline IA856 & 0.8565 & 0.0379 & 0.011 & 1.2 & 25.730 & 0.67 & 24.6 \\
\hline IRAC1 & 3.5569 & 0.7139 & 0.000 & 3.0 & 21.581 & 1.7 & 24.8 \\
\hline IRAC2 & 4.5020 & 0.9706 & 0.000 & 3.0 & 21.581 & 1.7 & 24.8 \\
\hline IRAC3 & 5.7450 & 1.3591 & 0.000 & 3.0 & 20.603 & 1.9 & 23.0 \\
\hline IRAC4 & 7.9158 & 2.7893 & 0.000 & 3.0 & 21.781 & 2.0 & 23.0 \\
\hline
\end{tabular}

Notes.

a $95 \%$ cumulative throughput width.

${ }^{\mathrm{b}}$ Median $5 \sigma$ depth calculated from the errors of objects in the final catalogs.

O. Almaini et al. (in preparation). UKIDSS uses the UKIRT Wide Field Camera (WFCAM; Casali et al. 2007. The photometric system is described in Hewett et al. (2006), and the calibration is described in Hodgkin et al. (2009). The pipeline processing and science archive are described in M. J. Irwin et al. (in preparation) and Hambly et al. (2008). We include the IRAC data from the Spitzer Public Legacy Survey of the UKIDSS UDS (SpUDS, PI: J. Dunlop et al.) in the 5.8 and $8 \mu \mathrm{m}$ pass bands and the SEDS v1.2 data release for the 3.6 and $4.5 \mu \mathrm{m}$ pass bands (Ashby et al. 2013). The central wavelength of the filter, 95\% cumulative throughput width of the filter, dust attenuation from Galactic extinction, aperture used for photometry, image zero point, and average FWHM, and median $5 \sigma$ depth for each of the images are listed in Table 8.

\section{PHOTOMETRY}

We construct a WFC3-selected photometric catalog for each field as detailed below. This is done in the same manner and consistently across all five fields. Briefly, we use a noiseequalized combination of the three WFC3 bands $(F 125 \mathrm{~W}$, 
Table 8

UDS Imaging Data

\begin{tabular}{lccccccc}
\hline \hline Band & $\begin{array}{c}\lambda_{\text {central }} \\
(\mu \mathrm{m})\end{array}$ & $\begin{array}{c}\text { Width }^{\mathrm{a}} \\
(\mu \mathrm{m})\end{array}$ & $\begin{array}{c}A_{\lambda} \\
(\mathrm{mag})\end{array}$ & $\begin{array}{c}\text { Aperture } \\
(\operatorname{arcsec})\end{array}$ & $\begin{array}{c}\text { Zero Point } \\
(\mathrm{AB})\end{array}$ & $\begin{array}{c}\text { FWHM } \\
(\operatorname{arcsec})\end{array}$ & $\begin{array}{c}5 \sigma \text { Depth }^{\mathrm{b}} \\
(\mathrm{mag})\end{array}$ \\
\hline$u$ & 0.3828 & 0.0771 & 0.092 & 1.5 & 25.350 & 0.90 & 26.4 \\
$B$ & 0.4408 & 0.1084 & 0.081 & 1.2 & 34.706 & 0.78 & 27.4 \\
$V$ & 0.5470 & 0.0993 & 0.061 & 1.2 & 33.602 & 0.83 & 27.2 \\
$F 606 W$ & 0.5921 & 0.2225 & 0.056 & 0.7 & 26.491 & 0.11 & 26.8 \\
$R$ & 0.6508 & 0.1194 & 0.048 & 1.2 & 34.260 & 0.79 & 26.9 \\
$i$ & 0.7655 & 0.1524 & 0.037 & 1.2 & 34.055 & 0.81 & 26.7 \\
$F 814 W$ & 0.8057 & 0.2358 & 0.034 & 0.7 & 25.943 & 0.11 & 26.8 \\
$z$ & 0.9060 & 0.1402 & 0.028 & 1.2 & 32.743 & 0.80 & 25.9 \\
$F 125 W$ & 1.2471 & 0.2867 & 0.016 & 0.7 & 26.230 & 0.18 & 25.8 \\
$J$ & 1.2502 & 0.1599 & 0.016 & 1.0 & 30.931 & 0.73 & 25.1 \\
$F 140 W$ & 1.3924 & 0.3760 & 0.013 & 0.7 & 26.452 & 0.18 & 25.2 \\
$H$ & 1.6360 & 0.2972 & 0.010 & 1.0 & 31.379 & 0.76 & 24.3 \\
$F 160 W$ & 1.5396 & 0.2744 & 0.011 & 0.7 & 26.946 & 0.19 & 25.9 \\
$K$ & 2.2060 & 0.3581 & 0.007 & 1.0 & 31.893 & 0.70 & 24.9 \\
IRAC1 & 3.5569 & 0.7139 & 0.000 & 3.0 & 21.581 & 1.7 & 24.6 \\
IRAC2 & 4.5020 & 0.9706 & 0.000 & 3.0 & 21.581 & 1.7 & 24.4 \\
IRAC3 & 5.7450 & 1.3591 & 0.000 & 3.0 & 21.581 & 1.9 & 21.7 \\
IRAC4 & 7.9158 & 2.7893 & 0.000 & 3.0 & 21.581 & 2.0 & 21.5 \\
\hline
\end{tabular}

Notes.

a $95 \%$ cumulative throughput width.

${ }^{\mathrm{b}}$ Median $5 \sigma$ depth calculated from the errors of objects in the final catalogs.

$F 140 W$ and $F 160 W$ ) for detection. We convolve each of the $H S T$ images to the same point-spread function (PSF) in order to measure consistent colors across multiple passbands. Aperture photometry was performed on the PSF-matched images in an aperture of 0.7 using SExtractor v2.8.6 (Bertin \& Arnouts 1996) in dual-image mode. For the ground-based and Spitzer data we used the photometry code Multi-resolution Object PHotometry oN Galaxy Observations (MOPHONGO) described in Labbé et al. (2006); Wuyts et al. (2007); Whitaker et al. (2011) to take into account the large difference in PSF size and confusion due to neighboring sources. A combination of the PSF-matched WFC3 images is used as a high resolution prior. We measure the aperture flux in each band, with an aperture size that depends on the PSF full-width at half maximum (FWHM) for each filter, and make a correction to total flux based on the $F 160 \mathrm{~W}$ growth curve. The methods we use are described below; they are similar to those described in Whitaker et al. (2011).

\subsection{Background Subtraction}

We determine the background level as a function of position across each image. For the HST images, we found that an initial run of SExtractor with the AUTO background subtraction using a background mesh size of 64 and filter size of 5 produced a reasonably flat background-subtracted image. We note that careful background subtraction for individual WFC3 pointings was already done as part of the reduction process described above; this additional step serves to remove any large scale gradients over the entire mosaic. For the ground-based and IRAC data, we applied a custom-developed code that uses a similar algorithm to mask sources and fit the background level in a variable-sized mesh, using sigma clipping to reduce the impact of bright sources. We verified that the flux distribution in empty regions of the image was centered on zero after the background subtraction.

\subsection{Source Detection}

For each field we create a noise-equalized version of the mosaic in each of the three WFC3 bands $F 125 W, F 140 W$ and $F 160 \mathrm{~W}$ by multiplying the science image by the square root of the inverse variance map. The three noise-equalized images are then coadded to form a deep detection image. As the variable weight across the mosaic is already taken into account with this method, we do not input a weight map to SExtractor when detecting sources on these images. We use a detection and analysis threshold of $1.8 \sigma$ and require a minimum area of 14 pixels for detection. The deblending threshold is set to 32 , with a minimum contrast parameter of 0.005 for all fields except GOODS-S, where we use a minimum contrast parameter of 0.0001 to improve the detection of sources within the wings of bright objects, particularly affecting the deep HUDF area. A Gaussian filter of 4 pixels is used to smooth the images before detection. The detection parameters were chosen as a compromise between deblending neighboring galaxies and splitting large objects into multiple components. An alternative approach would be to run SExtractor in a "hot" and "cold" mode, as was been done by the GEMS survey (Rix et al. 2004), and more recently, CANDELS (Guo et al. 2013; Galametz et al. 2013).

\subsection{HST PSF-Matching}

We PSF-match all the HST ACS and WFC3 images to the $F 160 W$ image, which has the PSF with the largest FWHM, before performing aperture photometry. An empirical PSF was created for each HST image by stacking isolated unsaturated stars from across the mosaic. As discussed in Section 2.1.2, we chose drizzle parameters that avoid clipping the centers of bright stars.

To create PSFs for each of the HST mosaics we made a careful selection of stars based on the tight stellar sequence in the ratio of the flux in a small aperture $\left(0^{\prime} .5\right)$ to that in a large aperture $\left(2^{\prime \prime}\right)$, adjusting the selection criteria appropriately for each of the ACS and WFC3 bands. The number of stars selected varied between 35 for the $F 606 \mathrm{~W}$ band in GOODS-S, which has limited coverage, to $\sim 200$ in $F 814 W$ in COSMOS. We mask neighboring objects within a postage stamp cut-out of 84 pixels $\left(\sim 5^{\prime \prime}\right)$ around each star. The postage stamps are recentered, normalized and then averaged to determine the PSF. Stamps where half-integer or large shifts are required to recenter the star are excluded from the final stack. Finally, we subtract a background correction based on the level measured in the outskirts ( $r>67$ pixels, corresponding to $\left.\sim 4^{\prime \prime}\right)$ of the stacked PSF stamp. We have not attempted to take variation with chip position into account, as the mosaics are made up of multiple pointings with different orientations and overlap, but such differences are likely to be small.

The PSF stamps for the ACS $F 814 W$ band and the WFC3 $F 160 \mathrm{~W}$ band in AEGIS are shown in Figure 9. PSFs for all the other fields and bands are shown in Appendix A. The PSFs in the figure are shown at three contrast levels for each of the ACS and WFC3 filters to expose the different level of structure: the core of the PSF, the first Airy ring $(\sim 0.5 \%)$ and the diffraction spikes $(\sim 0.1 \%)$. For a single orientation the HST PSF has four diffraction spikes, caused by the vanes of the secondary mirror assembly. The large number of diffraction spikes in the PSFs of Figure 9 (especially in the WFC3 PSFs) is due to the varying orientations of the data that went into the mosaics. We also show weight images, which encode the sum of the weights that went into each pixel.

The curve of growth (fraction of light enclosed as a function of aperture size) for each of the $F 160 \mathrm{~W}$ PSFs, normalized at $2^{\prime \prime}$, is shown in the top panel of Figure 10. The PSFs for the five fields are very consistent with each other, with almost indistinguishable growth curves on this scale. The agreement with the encircled energy as a function of aperture provided in the WFC3 

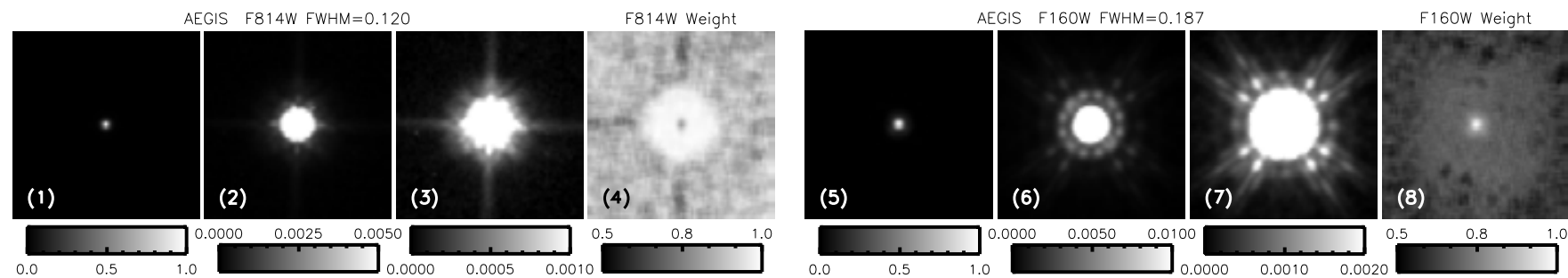

Figure 9. Point-spread functions (PSFs) for the ACS $/ F 814 \mathrm{~W}$ band and the WFC $3 / F 160 \mathrm{~W}$ band in the AEGIS field. The construction of the PSFs is described in Section 3.3. For each filter we show three stretch levels (panels 1-3 and 5-7) to expose the structure of the PSF: the core, the first Airy ring and the diffraction spikes. The images are normalized to a maximum value of one. The grayscale bars show the stretch for each panel. These are slightly different for ACS and WFC 3 as a result of the different FWHMs (listed above the images). We also show the combined weight images for each PSF. The weight is largest in the center and lower at larger radii due to masking of neighboring objects. The ACS PSFs have lower weights in the central pixels because of cosmic ray rejection flagging the centers of stars. PSFs for all other bands and fields are shown in the Appendix.

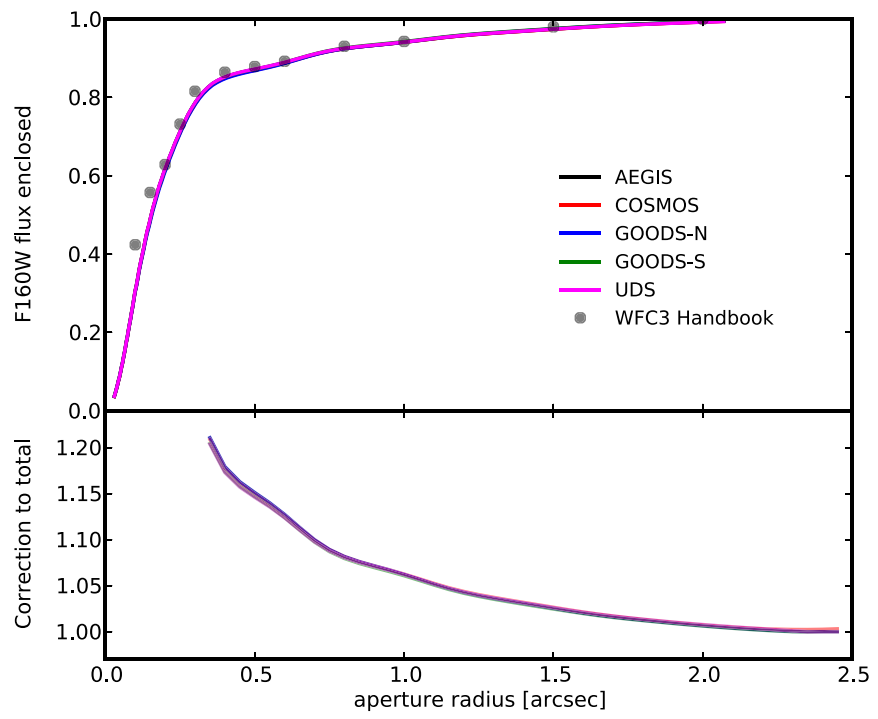

Figure 10. $F 160 \mathrm{~W}$ growth curves. Upper panel: the fraction of light enclosed as a function of radius relative to the total light within $2^{\prime \prime}, f(r) / f\left(2^{\prime \prime}\right)$, from the $F 160 W$ PSF stamp of each field. The PSFs of the five fields are very consistent with each other. The gray points show the encircled energy as a function of aperture size, also normalized to $2^{\prime \prime}$, from the WFC 3 handbook. The empirical growth curves agree well with the theoretical expectation. Lower panel: the correction to total flux for a point source with a circularized Kron radius equal to the aperture radius on the $x$-axis, derived as the inverse of the growth curves in the upper panel $\left(f\left(2^{\prime \prime}\right) / f(r)\right)$. The minimum Kron radius is set to the aperture radius in which we measure colors, 0 '. 35 , giving rise to a maximum correction of $\sim 1.21$.

(A color version of this figure is available in the online journal.)

handbook (normalized to the same maximum radius of $2^{\prime \prime}$ ) is also excellent. We show a comparison of the growth curves for the PSFs used in this paper to those generated from the CANDELS v1.0 mosaic and the "hybrid" PSF used for the morphological analysis in van der Wel et al. (2012) in Appendix A.

We use a deconvolution code developed by I. Labbé, which fits a series of Gaussian-weighted Hermite polynomials to the Fourier transform of the stacked stars, to find the kernel that convolves each PSF to match the $F 160 W$ PSF. We found residuals a factor of 5-10 times lower using this method than with the standard maximum entropy-type algorithms (e.g., the task lucy in IRAF). In Figure 11 we show the ratio of the growth curve in each band to that of the $F 160 \mathrm{~W}$ growth curve, before and after the convolution. The PSF-matching is accurate to $1 \%$ within a 0.7 aperture for all HST bands and fields.

\subsection{HST Photometry}

We ran SExtractor in dual mode to obtain aperture flux measurements for each HST band, using the detection images de- scribed above, the PSF-matched HST image and the corresponding weight map. We did not do a further background subtraction. Aperture photometry was done in an aperture of diameter 0 '.7 $^{\prime}$ in all the HST bands. Additional measurements in apertures of $1^{\prime \prime}, 1^{\prime \prime} .2,1^{\prime \prime} .5$ and $3^{\prime \prime}$ were done on the $F 140 W$ and $F 160 W$ images in order to correct the flux measurements from the ground and Spitzer bands to an equivalent color aperture, as described below.

The total flux in the reference band, which is chosen to be $F 160 \mathrm{~W}$ where there is $F 160 \mathrm{~W}$ coverage ( $99.7 \%$ of objects) and $F 140 \mathrm{~W}$ otherwise, is determined by correcting the SExtractor AUTO flux for the approximate amount of light that falls outside the AUTO aperture for a point source. This amount was calculated from the growth curves described in the previous Section. ${ }^{30}$ The AUTO flux is measured within a flexible elliptical aperture, known as the Kron radius (Kron 1980), that typically encloses $90 \%-95 \%$ of the total light. The correction is the inverse of the fraction of light within a circular aperture enclosing the same area as the Kron aperture (the circularized Kron radius), determined from the empirical growth curve for $F 160 \mathrm{~W}$.

$$
f_{\mathrm{WFC} 3 \text {, tot }}=f_{\mathrm{WFC} 3, \mathrm{AUTO}} \frac{f\left(r_{\mathrm{tot}}\right)}{f\left(r<r_{K}\right)},
$$

where $f\left(r<r_{K}\right)$ is the flux enclosed within the circularized Kron radius and $f\left(r_{\text {tot }}\right)$ is the total flux for a point source in $F 160 \mathrm{~W}$. The growth curve is normalized at $r_{\text {tot }}=2^{\prime \prime}$. We enforce a minimum radius of 0.35 , corresponding to the color aperture used for the HST photometry. The AUTO flux for objects with radii smaller than this is taken to be the flux measured in the color aperture.

The errors returned by SExtractor are known to be underestimated due to the correlations between pixels introduced by the drizzling process (Casertano et al. 2000). Rather than applying a single correction factor based on the drizzle parameters to all the errors, we determine the error in total magnitude and the errors on the HST aperture magnitudes by measuring the flux within empty apertures, as described by Labbé et al. (2005), Quadri et al. (2007), and Whitaker et al. (2011). In some of the $H S T$ bands, the depth varies dramatically across the image, so we cannot use an average measurement to represent the error at every point. To take variable depth into account, we measure the empty aperture fluxes on the noise-equalized mosaics. Each pixel in the noise-equalized mosaic is essentially weighted by

\footnotetext{
30 As is well known, these corrections slightly underestimate the required correction as galaxies have more extended profiles than point sources outside of the AUTO aperture. For our data these errors are small, as the total GALFIT-derived magnitudes of galaxies correspond very well to our total magnitudes (see Section 4.2).
} 

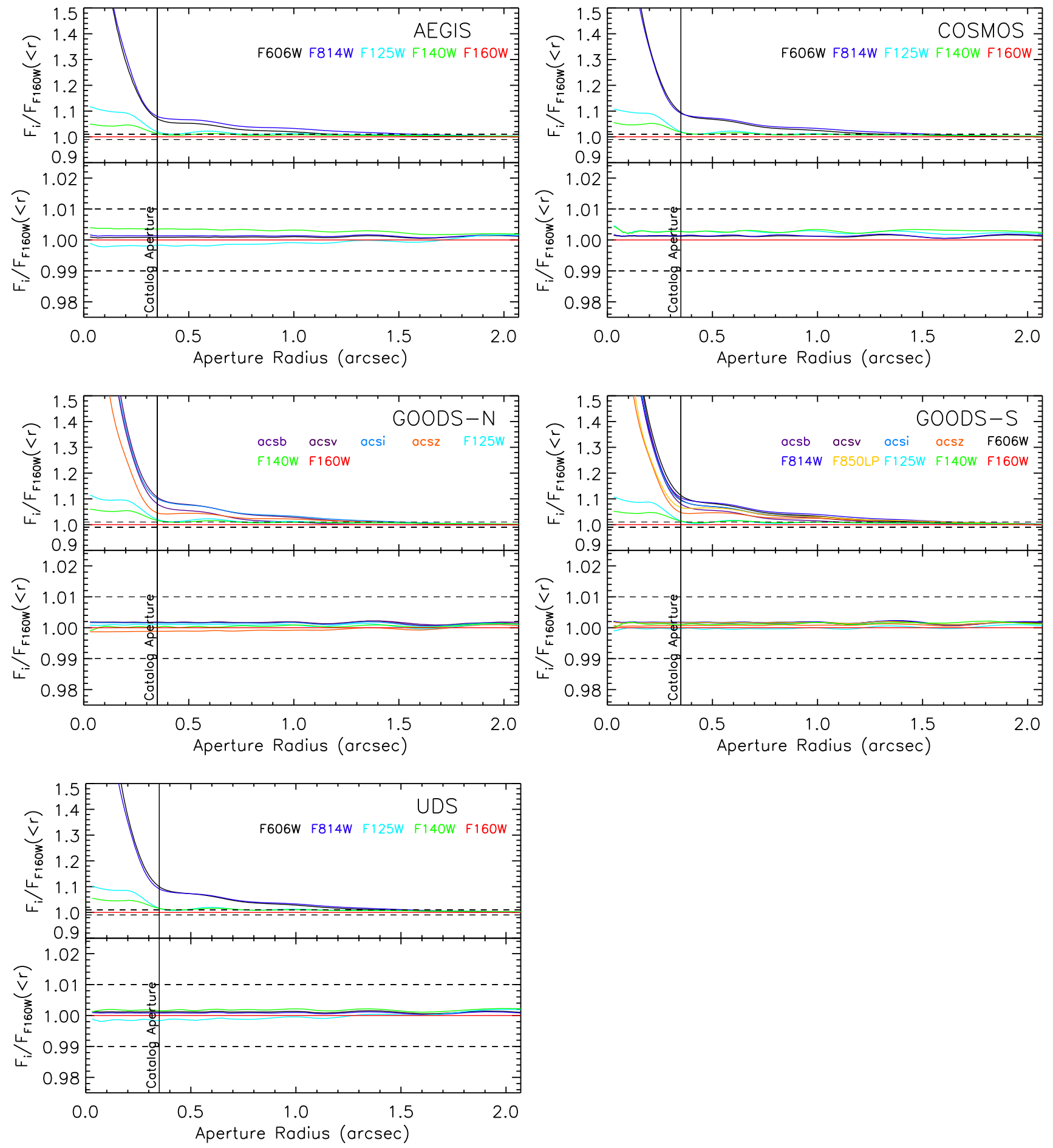

Figure 11. Growth curves showing the fraction of light enclosed as a function of radius for each HST filter relative to the $F 160 \mathrm{~W}$ growth curve in each of the five 3D-HST fields. The upper and lower panels show the growth curves before and after convolution to match the $F 160 \mathrm{~W}$ PSF, respectively. Note the change in scale between the upper and the lower panels. The dashed lines in both panels represents a $1 \%$ difference. After PSF-matching, the resulting growth curves in all bands are consistent with the $F 160 \mathrm{~W}$ PSF to well within $1 \%$ in each of the fields.

(A color version of this figure is available in the online journal.)

depth, bringing the noise to a standard level. We divide the resulting empty aperture errors by the square root of the weight at the position of each object for each band to obtain the errors provided in the catalogs.

To estimate the error on the total magnitudes, we determine the background noise in an aperture the size of the circularized Kron radius for each object, and correct this to total in the same way as for the fluxes, described by Equation (1) above.
To determine how the background noise scales with aperture size, we measure the distribution of counts in empty regions of increasing size within the noise-equalized $F 160 W(F 140 W)$ image. For each aperture size we measure the flux in $>2000$ apertures placed at random positions across the image. We exclude apertures that overlap with sources in the detection segmentation map. As an example, the left hand panel of Figure 12 shows the distribution of flux counts in empty 

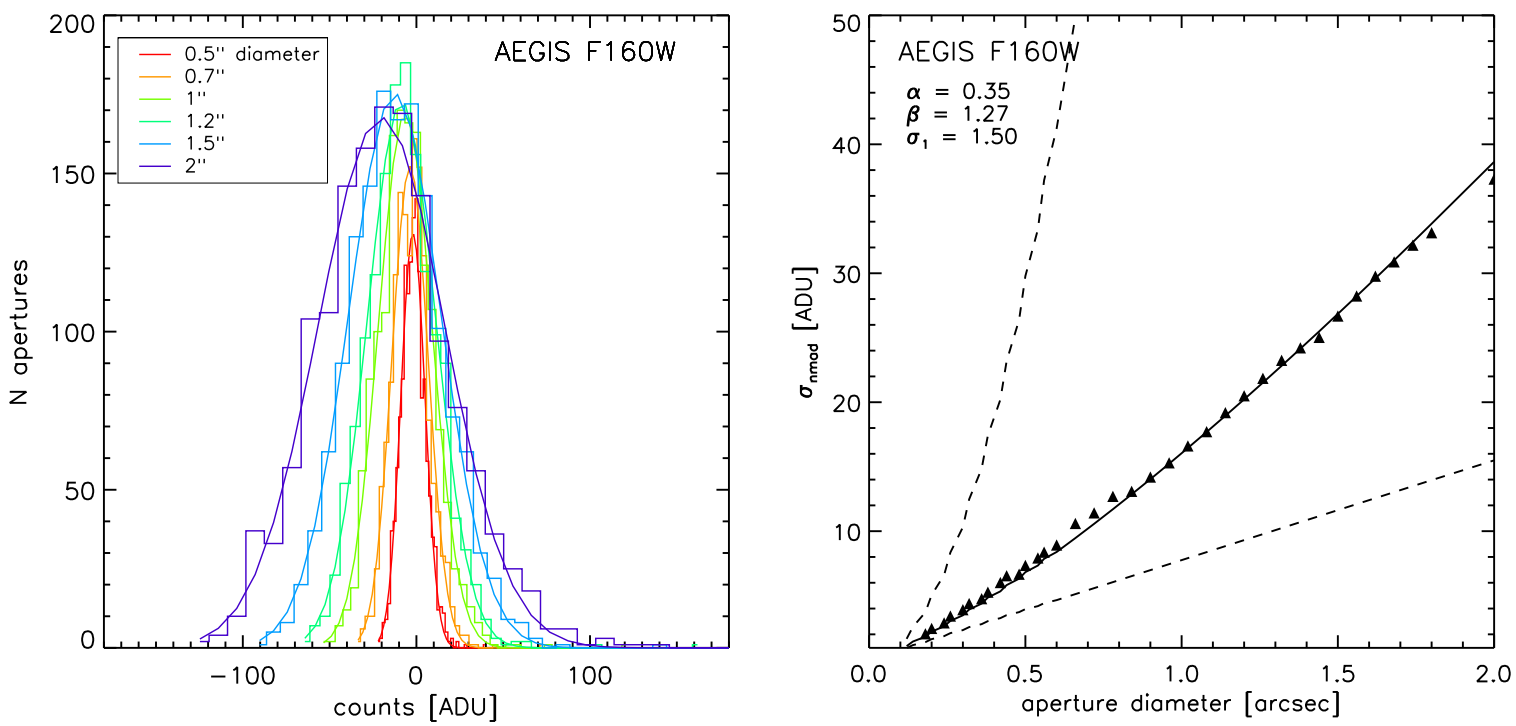

Figure 12. Histograms of summed counts in different aperture sizes from empty regions throughout the image (left-hand panel) and the resultant noise-scaling as a function of aperture (right-hand panel) for the AEGIS $F 160 \mathrm{~W}$ image. The measured $\sigma_{\text {nmad }}$ are shown by the triangles. The solid line shows the power-law fit to the data, with the fit parameters given in the upper left. The dashed lines show the linear $(\propto N)$ and $N^{2}$ scalings, which correspond to no correlation and perfect correlation between the pixels, respectively.

(A color version of this figure is available in the online journal.)

Table 9

Power-Law Parameters for Empty Aperture Errors

\begin{tabular}{llc}
\hline \hline Field & $\alpha$ & $\beta$ \\
\hline AEGIS & 0.35 & 1.27 \\
COSMOS & 0.35 & 1.28 \\
GOODS-N & 0.3 & 1.31 \\
GOODS-S & 0.3 & 1.31 \\
UDS & 0.3 & 1.31 \\
\hline
\end{tabular}

apertures of $0.5,0.7,1,1.2,1.5$, and $2^{\prime \prime}$ diameter in the UDS noise-equalized $F 160 \mathrm{~W}$ image. The other fields show similar distributions. Each histogram can be well-described by a Gaussian, with the width increasing as aperture size increases. The increase in standard deviation with linear aperture size $N=\sqrt{A}$, where $A$ is the area within the aperture, can be described as a power law. A power-law index of 1 would indicate that the noise is uncorrelated, while if the pixels within the aperture were perfectly correlated, the background noise would scale as $N^{2}$. The right-hand panel of Figure 12 shows the measured standard deviation as a function of aperture size in the UDS noise-equalized $F 160 \mathrm{~W}$ image. We fit a power-law of the form

$$
\sigma=\sigma_{1} \alpha N^{\beta}
$$

where $\sigma_{1}$ is the standard deviation of the background pixels, fixed to a value of 1.5 here, $\alpha$ is the normalization and $1<\beta<2$ (see Whitaker et al. 2011). The fitted parameters for each field are listed in Table 9. The power-law fit is shown by the solid line in the figure. The bracketing $\beta=1$ and $\beta=2$ scalings are shown by the dashed lines. The final error in the catalog is divided by the square root of the weight at the position of the object, as described above. Errors in the aperture fluxes are similarly calculated by measuring the standard deviation of the flux distribution in apertures of 0.7 diameter on the noiseequalized $H S T$ images.

We note that the distributions for the largest apertures show that the background flux is slightly negative far away from objects. This systematic error in the background is much smaller than the random error. We did not correct for this error as it is very small compared to the quoted errors and as it is not clear whether the background is similarly affected in regions where objects are detected.

\subsection{Low-Resolution Data Photometry}

The large differences between the PSF sizes in the HST data and the ground-based and Spitzer data must be taken into account in order to obtain accurate color information, without degrading the HST images to lower $\mathrm{S} / \mathrm{N}$ through smoothing or losing the exquisite high resolution information they provide. We use the MOPHONGO code developed by one of us (I. Labbé) to perform the photometry on the ground-based and Spitzer IRAC images, as described in Labbé et al. (2006), Wuyts et al. (2007), and Whitaker et al. (2011).

The code uses a high-resolution image as a prior to estimate the contributions from neighboring blended sources in the lower resolution image. We use an average of the PSF-matched $F 125 W, F 140 W$ and $F 160 W$ images as the high-resolution prior. A shift map that captures small differences in the astrometry of the high resolution reference image and low resolution measurement image is created by cross-correlating the object positions in the two images. The position-dependent convolution kernel that maps the higher resolution PSF to the lower resolution PSF is determined by fitting a series of Gaussianweighted Hermite polynomials to the Fourier transform of a number of point sources across each image. Point sources are selected in the same way as for the HST PSF-matching described above. Poorly fit objects are rejected and a smoothed map of the appropriate coefficients is created in two iterations. The high resolution image is then convolved with the local kernel to obtain a model of the low resolution image, with the flux normalization of individual sources as a free parameter. Photometry in an aperture size appropriate to the size of the PSF is done on the original image, with a correction applied for contamination from neighboring sources around each object as determined from the model. Fluxes are further corrected to account for flux that falls 
outside of the aperture due to the large PSF size. The correction is given by the ratio of the flux enclosed in the photometric aperture in the high resolution image (before convolution) to the low-resolution model (after convolution).

For the ground-based NIR and optical images we use aperture diameters that depend on the seeing. For images that have FWHM $\leqslant 0.8$ we use an aperture of $1^{\prime \prime}$, for images with $0^{\prime \prime} 8<$ FWHM $\leqslant 1^{\prime \prime}$. 0 we use an aperture of $1^{\prime \prime} .2$, and for images with larger FWHM we use an aperture of 1".5. For the optical medium bands in GOODS-S we use a minimum aperture of $1^{\prime \prime}$ and an aperture of $3^{\prime \prime}$ for the few bands with seeing $>1^{\prime \prime} .5$. For the IRAC bands we use an aperture diameter of $3^{\prime \prime}$. The apertures, FWHM and image zero points are listed in Tables 4-8.

The GOODS-N and GOODS-S fields have two epochs of IRAC 5.8 and $8 \mu \mathrm{m}$ data that overlap in the center of the field. For these fields, we compute average of the two flux measurements for objects that have a weight greater than zero in both epochs, after correcting each flux to total as described below. The errors are added in quadrature and the weight given in the catalog is the average of the relative weights in the two epochs.

\subsection{Flux Corrections}

We correct for Galactic extinction using the values given by the NASA Extragalactic Database extinction law calculator ${ }^{31}$ at the center of each field, based on the recalibration by Schlafly \& Finkbeiner (2011) of the COBE/DIRBE and IRAS/ISSA dust maps (Schlegel et al. 1998). The extinction estimates assume a Fitzpatrick (1999) reddening law with $R_{V}=3.1$. We interpolate between the values provided for a small set of filters to determine the extinction at the central wavelength of each filter in our data set. We do not account for the variable width of the filters or variations across the field. The Galactic extinction corrections are generally small ( $\lesssim 0.05 \mathrm{mag}$ ). Tables $4-8$ list the corrections applied for each band.

The fluxes provided in the catalog are total fluxes. We correct the color flux measured in each band to a total flux by multiplying by the ratio of the $F 160 W(F 140 W)$ total flux to the $F 160 W(F 140 W)$ flux measured in the appropriately sized aperture:

$$
f_{X, \text { tot }}=f_{X}(r) \times \frac{f_{\mathrm{WFC} 3, \mathrm{tot}}}{f_{\mathrm{WFC} 3}(r)},
$$

where $X$ represents each filter, $r$ is the appropriate aperture for that band as given in Tables $4-8$, and $f_{\mathrm{WFC} 3 \text {,tot }}$ is the total flux in the reference band as calculated by Equation (1). Note that differences between the PSFs have already been taken into account, so that $f_{X}(r)$ and $f_{\mathrm{WFC} 3}(r)$ measure an equivalent fraction of the total light in different bands. The aperture errors are similarly converted to a total error by multiplying by the same correction as the fluxes.

The $F 160 W$ and $F 140 W$ aperture flux measured within 0.7 as well as the total $F 160 \mathrm{~W}$ and $F 140 \mathrm{~W}$ flux are provided for each object in the catalog, allowing one to convert back to a consistent color measurement for any band. The correction from AUTO to total is also provided as a column in the catalog. An addition correction is applied to all the catalog fluxes to account for zero point and template uncertainties, as listed in Table 11. This is described in detail in Section 5 below.

\subsection{Point Source Classification}

Compact or unresolved sources form a tight sequence in sizemagnitude space, with fairly constant, small sizes as a function

\footnotetext{
31 http://ned.ipac.caltech.edu/help/extinction_law_calc.html
}

of magnitude. In the left panel of Figure 13 we show the SExtractor FLUX_RADIUS against total $F 160 W$ magnitude for the GOODS-N field. Point sources can be cleanly separated from extended sources down to $H_{F 160 W} \sim 25 \mathrm{mag}$. We provide a point source flag in the catalog based on cuts in this space, as measured on the $F 160 \mathrm{~W}$ images. Objects are classified as point sources (star_flag $=1$ ) if they have $H_{F 160 \mathrm{~W}} \leqslant 25 \mathrm{mag}$ and FLUX_RADIUS $<-0.115 H_{F 160 W}+5.15$. These objects are shown as red stars in the figure. Note that the group of very compact objects lying well below the stellar sequence are mostly artifacts. Objects fainter than 25 mag (dotted red line) cannot be cleanly separated and are assigned a star_flag of 2. All other objects are classified as extended, with star_flag $=0$. For the small fraction of objects with no coverage in $F 160 \mathrm{~W}$, we assign a star_flag of 2 .

We note that the ratio of fluxes in a large $\left(2^{\prime \prime}\right)$ and small $(0 ! 5)$ aperture plotted against magnitude provides a similar tight sequence of point sources for $H_{F 160 \mathrm{~W}} \lesssim 24$ mag (right hand panel of Figure 13). A selection in this space was made for the PSF-matching. Both sequences proved to be useful diagnostics of the image quality, with large spread in the stellar sequence indicating that the centers of stars have been down weighted during cosmic ray rejection.

\section{8. "Use" Flag}

For convenience, we provide a flag with the catalog that allows a straightforward selection of galaxies that have photometry of reasonably uniform quality. This "use" flag (listed as "use_phot" in the catalog, to distinguish it from spectroscopic quality flags) is set to 1 if the following criteria are met:

1. Not a star, or too faint for reliable star/galaxy separation: star_flag $=0$ or star_flag $=2$.

2. Not close to a bright star. The halos and diffraction spikes of bright stars can cause severe problems with the photometry, in particular in ground-based optical images. This criterion is implemented in two parts: not within $18^{\prime \prime}$ of a star with $F 606 W<17$ and/or $F 160 W<15$, and not within $12^{\prime \prime}$ of a star with $F 606 W<19$ and/or $F 160 W<17$. This near_star flag is provided as a catalog column.

3. Well-exposed in the $F 125 W$ and $F 160 W$ bands. We require that a minimum of two individual exposures cover the object in both $F 125 W$ and $F 160 W$. This removes objects on the edges of the mosaics, and in gaps. The number of exposures for a given object is the median number in a $0.7 \times 0.7$ $(12 \times 12$ pixel $)$ box. The number of exposures in each of the WFC3 bands are provided as catalog columns.

4. A detection in $F 160 W$. We apply a very low $\mathrm{S} / \mathrm{N}$ cut to limit the number of false positives, requiring f_F $F 160 W /$ e_ $F 160 W>3$.

5. A "non-catastrophic" photometric redshift fit (see Section 5.2). The criterion is $\chi_{p}<1000$.

6. A "non-catastrophic" stellar population fit (see Section 5.4). This criterion is $\log (M)>0$.

This flag selects approximately $85 \%$ of all objects in the catalogs. The flag is not very restrictive: for most science purposes further cuts (particularly on magnitude or $\mathrm{S} / \mathrm{N}$ ratio) are required. Furthermore, we caution that the flag is not $100 \%$ successful in weeding out problematic SEDs. The overall quality of the SEDs is higher for galaxies with a higher $\mathrm{S} / \mathrm{N}$ in the WFC3 bands. Although the use_phot flag only requires a $\mathrm{S} / \mathrm{N}>3$, we caution that the SEDs of galaxies with $\mathrm{S} / \mathrm{N}$ $(F 160 W) \lesssim 7$ will be quite noisy. As with all photometric 

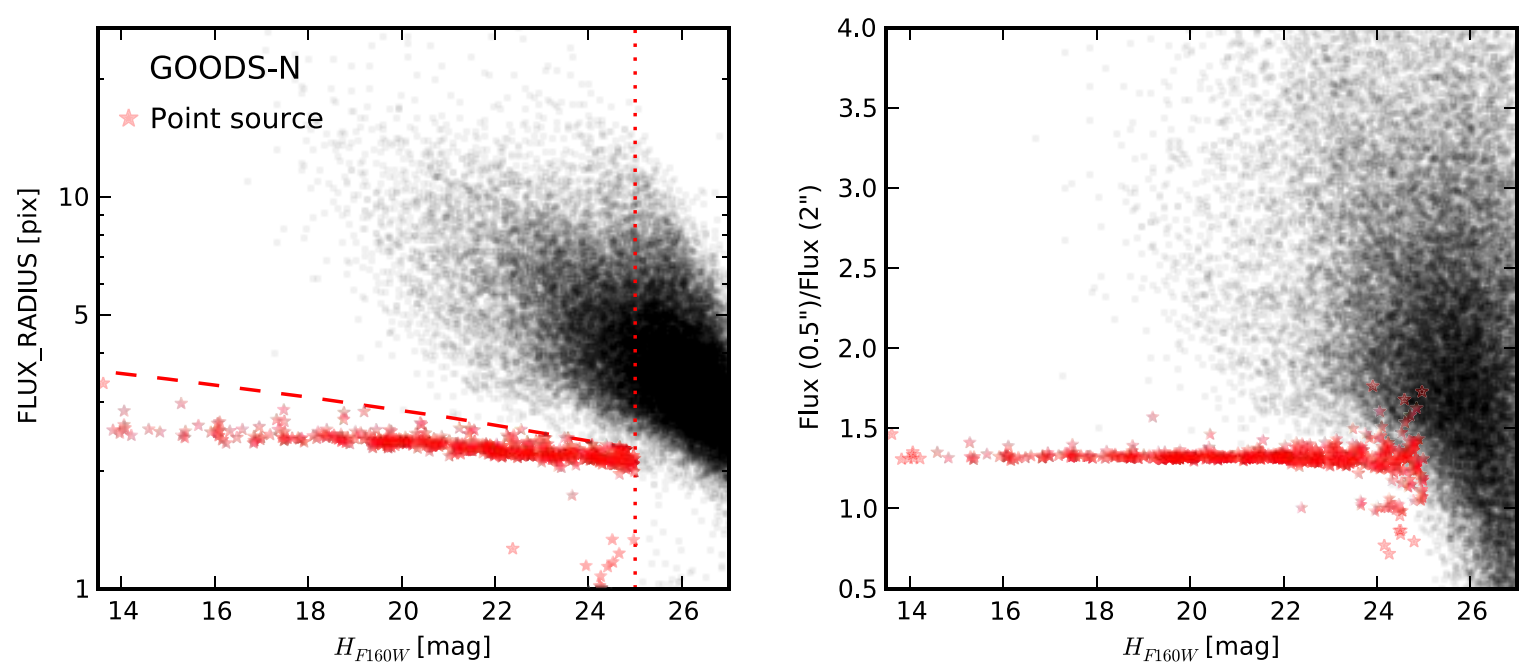

Figure 13. Left panel: SExtractor's FLUX_RADIUS against total $F 160 \mathrm{~W}$ magnitude for the GOODS-N field. Objects classified as point sources in the catalog are shown with red star symbols, galaxies and uncertain classifications (with $H_{F 160 \mathrm{~W}}>25 \mathrm{mag}$ ) as black symbols. The red dashed line, corresponding to the equation in the text, is used to make the selection. The red dotted line shows the magnitude limit of 25 , beyond which the classification is deemed uncertain. The right panel demonstrates an alternate method of selecting point sources using the ratio of fluxes in a large and small aperture. The tightness of the stellar sequence in this ratio at brighter magnitudes $\left(H_{F 160 \mathrm{~W}} \lesssim 24 \mathrm{mag}\right)$ allows for a more stringent classification, but the separation becomes less clear than the flux radius selection at fainter magnitudes. The flux ratio was used to select stars for the PSF-matching and kernel fitting. The symbols in the right panel correspond to the selection shown in the left panel; in general, the two methods agree well.

(A color version of this figure is available in the online journal.)

catalogs, individual SEDs should be inspected when selecting objects for, say, spectroscopic follow-up studies. For statistical studies of large samples the use_phot flag should be sufficiently reliable, particularly when combined with a $\mathrm{S} / \mathrm{N}$ or magnitude criterion.

\subsection{Catalog Format}

We provide a full photometric catalog for each of the five 3D-HST fields, as well as a master catalog with a subset of parameters in common for objects from all five fields. The catalogs contain total flux measurements and structural parameters for 207967 objects in total: 41200, 33879, 38279, 50507, and 44102 for AEGIS, COSMOS, GOODS-N, GOODS$\mathrm{S}$, and the UDS, respectively.

A description of the columns in each photometric catalog is given in Table 10. All fluxes are normalized to an AB zero point of 25 , such that

$$
\operatorname{mag}_{A B}=-2.5 \times \log _{10}(F)+25 .
$$

As described above, the catalogs contain the aperture flux in 0.7 and $1 \sigma$ error for the $F 140 W$ and $F 160 W$ bands and the total fluxes and $1 \sigma$ errors for every band listed in Table 3. The structural parameters from SExtractor and the corrections to total magnitudes are derived from the $F 160 \mathrm{~W}$ image where there is $F 160 \mathrm{~W}$ coverage and $F 140 \mathrm{~W}$ otherwise (only $0.3 \%$ of objects). The "f140w_flag" column indicates whether the $F 140 W$ image was used rather than $F 160 W(1=F 140 W$ used, $0=F 160 W$ used).

For the GOODS fields, where there is $H S T /$ ACS data from both the GOODS survey and CANDELS, we append "cand" to the column names for the CANDELS data. The NIR column names correspond to the filters listed in Tables $4-8$ and in some cases are appended with the survey name for clarity.

We provide a weight column for each band to indicate the relative weight for each object compared to the maximum weight for that filter. In practice, the weight is calculated as the ratio of the weight at each object's position to the 95th percentile of the weight map. We smooth the weight map using a filter of 3 pixels, and use the 95th percentile rather than the absolute maximum, to avoid being affected by extreme values. Objects with weights greater than the 95th percentile weight have a value of 1 in the weight column.

The catalog also contains a series of "minimum weight" columns (wmin_ground, wmin_hst, wmin_wfc3 and wmin irac) which store the minimum of the relative weight values for the set of ground-based, HST, HST/WFC3 and IRAC filters, respectively. These columns provide a useful way to select objects that have sufficient coverage in a particular set of filters. The "nexp_f125W," "nexp_f140w" and "nexp_f160W" columns in the catalogs give the median number of exposures for each of the WFC3 bands in a $12 \times 12$ pixel $\left(0^{\prime \prime} .7 \times 0^{\prime \prime} .7\right)$ box around each object.

\subsection{Completeness}

The depth of the images varies from field to field and across individual fields, as we show in the weight maps in Figures 2-6. As a result, the completeness will depend on position, as well as varying for sources of different morphologies, magnitudes and sizes. For a discussion of the completeness within the deep and wide areas within the CANDELS/GOODS-S field and the simulated dependence of completeness on size and flux for de Vaucouleurs and exponential galaxy profiles, see Guo et al. (2013).

The nominal completeness within the CANDELS/Wide survey images can be estimated by comparing the number of detections in a shallow image with the typical exposure time of the CANDELS/Wide survey (2 orbit depth) to those in the CANDELS/Deep area in the GOODS-South field, which has approximately four times the exposure time. This is the method that was used by Tal et al. (2014) to determine completeness as a function of various galaxy properties, such as redshift, mass, etc. We follow the same procedure here to determine the completeness as a function of $F 160 \mathrm{~W}$ magnitude. 
Table 10

Catalog Columns

\begin{tabular}{|c|c|}
\hline Column Name & Description \\
\hline id & Unique identifier \\
\hline$x$ & $\mathrm{X}$ centroid in image coordinates \\
\hline$y$ & Y centroid in image coordinates \\
\hline $\mathrm{ra}$ & RA J2000 (degrees) \\
\hline dec & Dec J2000 (degrees) \\
\hline faper_F $F 160 W$ & $F 160 \mathrm{~W}$ flux within a 0.7 arcsecond aperture \\
\hline eaper_F160W & 1 sigma error within a 0.7 arcsecond aperture \\
\hline faper_F140W & $F 140 \mathrm{~W}$ flux within a 0.7 arcsecond aperture \\
\hline eaper_F140W & 1 sigma error within a 0.7 arcsecond aperture \\
\hline $\mathrm{f} \_\mathrm{X}$ & Total flux for each filter $\mathrm{X}$ (zero point $=25$ ) \\
\hline$e_{-} X$ & 1 sigma error for each filter $\mathrm{X}$ (zero point $=25$ ) \\
\hline $\mathrm{w}_{-}^{-} \mathrm{X}$ & Weight relative to maximum exposure within image $\mathrm{X}$ (see text) \\
\hline tot_cor & Inverse fraction of light enclosed at the circularized Kron radius \\
\hline wmin_ground & Minimum weight for all ground-based photometry (excluding zero exposure) \\
\hline wmin_hst & Minimum weight for ACS and WFC 3 bands (excluding zero exposure) \\
\hline wmin_wfc3 & Minimum weight for WFC3 bands (excluding zero exposure) \\
\hline wmin_irac & Minimum weight for IRAC bands (excluding zero exposure) \\
\hline Z_spec & Spectroscopic redshift, when available (see notes on quality in each field) \\
\hline \multirow[t]{2}{*}{ star_flag } & Point source $=1$, extended source $=0$ for objects with total $H_{F 160 \mathrm{~W}} \leqslant 25 \mathrm{mag}$ \\
\hline & All objects with $H_{F 160 W}>25$ mag or no $F 160 W$ coverage have star_flag $=2$ \\
\hline kron_radius & SExtractor KRON_RADIUS (pixels) \\
\hline a_image & Semi-major axis (SExtractor A_IMAGE, pixels) \\
\hline b_image & Semi-minor axis (SExtractor B_IMAGE, pixels) \\
\hline theta $\_$J2000 & Position angle of the major axis (counter-clockwise, measured from East) \\
\hline class_star & Stellarity index (SExtractor CLASS_STAR parameter) \\
\hline flux_radius & Circular aperture radius enclosing half the total flux (SExtractor FLUX_RADIUS parameter, pixels) \\
\hline fwhm image & FWHM from a Gaussian fit to the core (SExtractor FWHM parameter, pixels) \\
\hline flags & SExtractor extraction flags (SExtractor FLAGS parameter) \\
\hline IRACx_contam & Ratio of contaminating flux from neighbors to the object's flux in each of the IRAC bands $(x=1-4)$ \\
\hline contam_flag & $\begin{array}{l}\text { A flag indicating if any of the photometry has a contamination ratio } \geqslant 50 \% \text { in any of the IRAC bands } \\
(1 \text { if } \geqslant 50 \% \text { in at least } 1 \text { band, } 0=\mathrm{OK})\end{array}$ \\
\hline f140w_flag & A flag indicating whether the corrections and structural parameters were derived from $F 140 \mathrm{~W}$ rather than $F 160 \mathrm{~W}$ \\
\hline use_phot & Flag indicating source is likely to be a galaxy with reliable measurements (see text) \\
\hline near_star & Flag indicating whether source is close to a star $(1=$ close to a bright star, $0=\mathrm{OK})$ \\
\hline nexp_f125w & Median number of exposures in $F 125 W$ within a $12 \times 12$ pixel box centered on the source \\
\hline nexp_f140w & Median number of exposures in $F 140 W$ within a $12 \times 12$ pixel box centered on the source \\
\hline nexp_f160w & Median number of exposures in $F 160 W$ within a $12 \times 12$ pixel box centered on the source \\
\hline
\end{tabular}

Note. $X=$ filter name, as given in Table 3, or IRAC1, IRAC2, IRAC3, IRAC4 representing the 3.6, 4.5, 5.8, and $8 \mu \mathrm{m}$ IRAC bands.

Deep and shallow $F 125 W$ and $F 160 W$ images of the central area of GOODS-S reduced in a similar way to that described in Section 2.1 above were kindly provided by D. Magee. We created new deep and shallow detection images by coadding the noise-equalized $F 125 \mathrm{~W}, F 140 \mathrm{~W}$ and $F 160 \mathrm{~W}$ images in the same way as described in Section 3.2 and applied the same SExtractor parameters to detect sources. We adjusted the DEBLEND_MINCONT parameter to 0.005 for the shallow image and 0.0001 for the deep image, as the detection of objects in the wings of nearby bright objects becomes problematic in the deeper image. We ran SExtractor in dual mode on each detection image, with our $F 160 \mathrm{~W}$ image as the measurement image. The number of detections in the shallow image are compared to the number of detections in the deep image as a function of $F 160 \mathrm{~W}$ AUTO magnitude in Figure 14 (green and gray histograms). The fraction of detections recovered in the shallow image compared to the deep image is shown by the thick black line. We find that the number of detections in the shallow image begins to deviate significantly from the deep image at an $H_{F 160 W}$ magnitude of 25, reaching $90 \%, 75 \%$ and $50 \%$ completeness levels at magnitudes of $25.1,25.9$ and $26.5 \mathrm{mag}$, respectively. Note that the decrease is fairly gradual at first, so the $75 \%$ completeness level we quote is more reliable than the $90 \%$ level.
We also compare the number of objects detected in the shallow image to the number expected based on the detections in the HUDF (53 orbit depth), using the catalog of Lundgren et al. (2014). As the HUDF is much smaller in area than the images we use for these tests, we have scaled the counts in the HUDF by a factor of nine to bring them into approximate agreement at the bright end. The scaled HUDF counts are shown by the red histogram in Figure 14. We find good agreement between the scaled HUDF counts and the number of detections in the deep image down to $H_{F 160 W} \sim 25.8 \mathrm{mag}$, but the HUDF is approximately $1 \mathrm{mag}$ deeper, resulting in a faster drop in the fraction of objects detected in the shallow image. The fraction of objects recovered in the shallow image relative to the HUDF reaches $90 \%, 75 \%$ and $50 \%$ at magnitudes of $25.1,25.8$ and $26.2 \mathrm{mag}$, respectively. This is shown by the dashed black line in Figure 14. The weight of the shallow image used here corresponds to the shallowest portions of each of the full mosaics and this is therefore a conservative estimate of completeness.

\subsection{Number Counts}

We determined the approximate effective survey area of each of the five fields in the following way. For each of the WFC3 


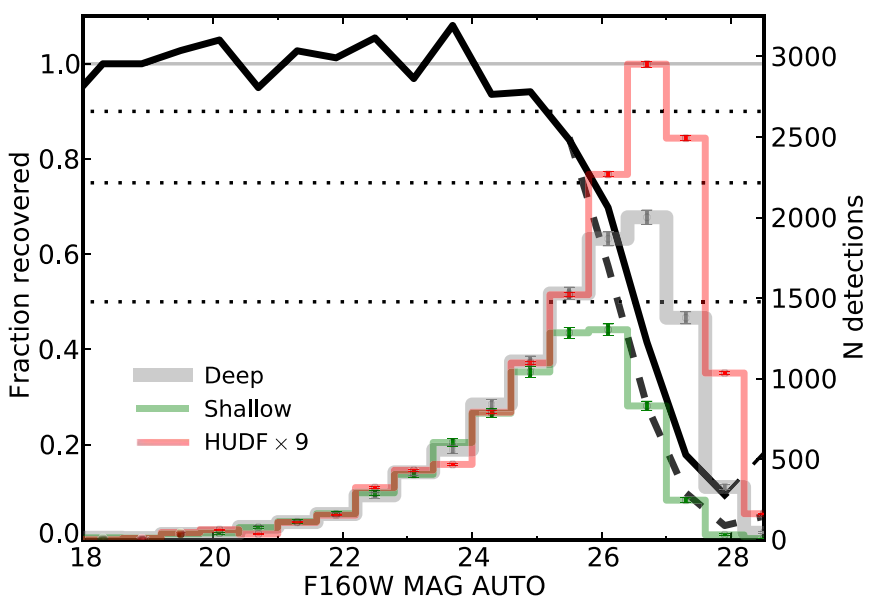

Figure 14. Completeness fraction as a function of $F 160 \mathrm{~W}$ magnitude determined by comparing the detections in an image with the typical depth of the CANDELS/Wide images to the CANDELS/Deep image of the same area within GOODS-South and the HUDF. The deep image is twice as deep as the shallow image, with approximately four times the exposure time. The counts in the HUDF have been scaled by a factor of nine to account for the difference in area. The solid black line shows the ratio of the counts in the shallow and deep areas. The fraction drops off faster relative to the HUDF, as seen from the dashed black line at $H_{160 W}>25$. The dotted lines show detection fractions of $90 \%, 75 \%$ and $50 \%$ with corresponding $H_{160 W}$-AUTO magnitudes of 25.1 , 25.9 and 26.5 mag relative to the deep image and 25.1, 25.8, and $26.2 \mathrm{mag}$ relative to the HUDF. The gray, green and red histograms show the number of objects detected in the deep, shallow and HUDF images, respectively, with the error bars representing Poisson errors.

(A color version of this figure is available in the online journal.)

images we create a map of the number of exposures contributing to each pixel. We masked out the areas affected by bright stars, using the near_star criteria described above. The useful science area (corresponding to the use_phot flag) was then calculated by adding up the number of unmasked pixels with at least two exposures in both $F 125 W$ and $F 160 W$. The maps are provided as part of the data release. The area of each field is given in Table 1. The number density of galaxies (satisfying our "use_phot" flag criterion) in the 3D-HST fields is shown in Figure 15. The left-hand panel shows the number counts as a function of total $H_{F 160 W}$ magnitude for each of the five fields, while the right hand panel compares the $K$-band number counts for AEGIS and COSMOS with those obtained by the NMBS (Whitaker et al. 2011) and the UltraVISTA catalog from Muzzin et al. (2013). The error bars represent Poisson errors in both panels. The number counts are fairly consistent across the five fields. COSMOS shows a slight excess of objects compared to other fields, particularly at the bright end. The NMBS found a similar difference between COSMOS and the AEGIS field, as can be seen in the right hand panel. For bright sources, the agreement between the two surveys in the AEGIS field is excellent, while for COSMOS, 3D-HST and UltraVISTA show excellent agreement but slightly lower number counts than NMBS.

\subsection{Photometry of Close Pairs}

We caution that the photometry of galaxies that are in close proximity of one another may have systematic errors that are not properly accounted for in the formal uncertainties. The groundbased and IRAC photometry is performed after subtracting a model for neighboring sources (see Section 3.5), but the space-based photometry is performed directly on PSF-matched data without explicitly accounting for the flux of neighbors. SExtractor does attempt to mask and correct the aperture fluxes symmetrically for regions affected by overlapping objects (with the MASK_TYPE parameter set to CORRECT). As described in Section 3.4 the photometric aperture has a diameter of 0 I. $^{\prime} 7$. We estimate the fraction of potentially affected objects in the

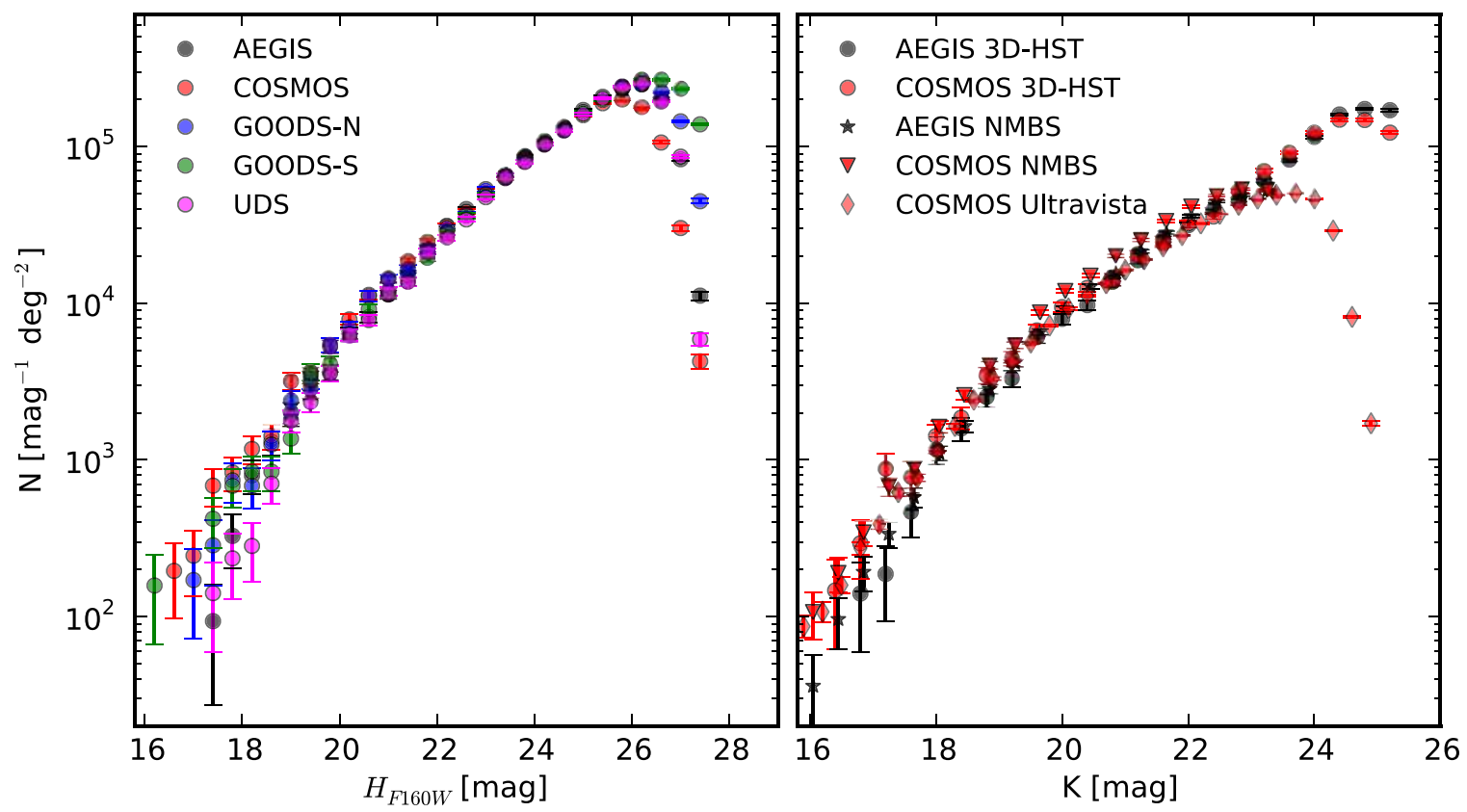

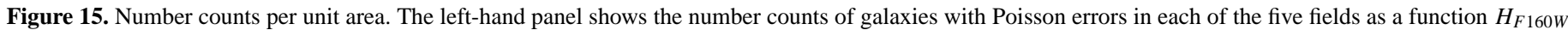

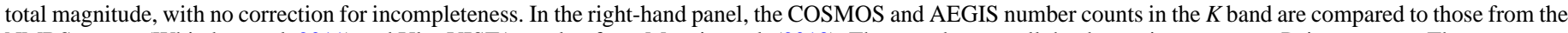

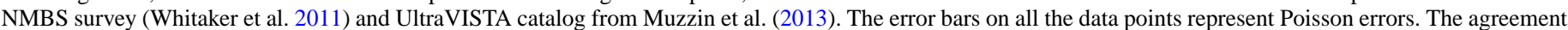

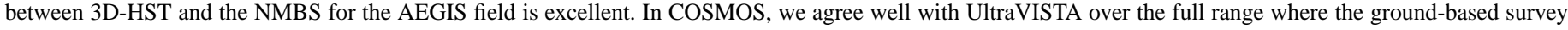

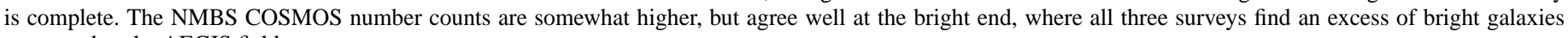
compared to the AEGIS field.

(A color version of this figure is available in the online journal.) 


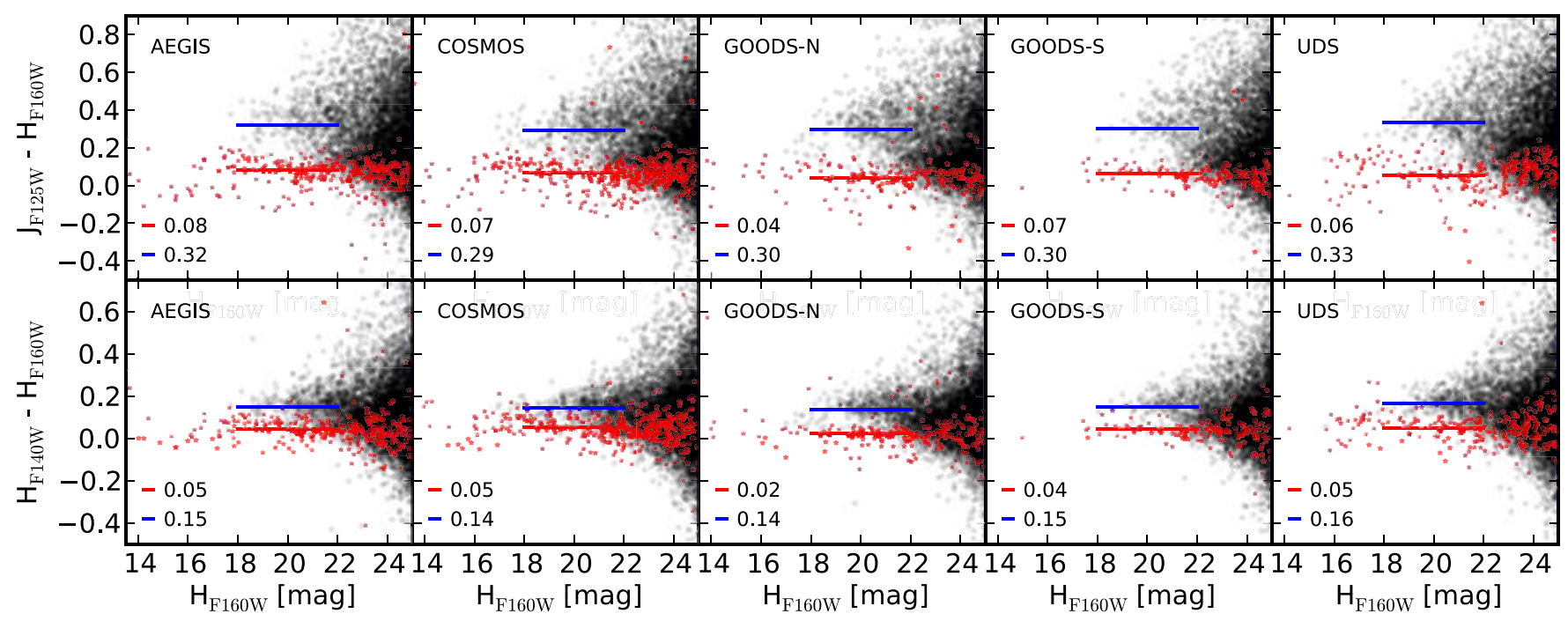

Figure 16. $J_{F 125 W}-H_{F 160 W}$ and $H_{F 140 W}-H_{F 160 W}$ colors vs. $H_{F 160 W}$ magnitude for each of the five fields. Point sources are shown in red and extended sources in black. The medians for point sources and extended sources in the range $18<H_{F 160 W}<22$ are labeled and shown by the red and blue lines, respectively. Objects with a SExtractor flag $>0$ have been excluded.

(A color version of this figure is available in the online journal.)

catalog by determining the number of pairs with a distance smaller than 0 '.7, i.e., with overlapping photometric apertures. This fraction ranges from $3 \%$ to $7 \%$ in the five fields, with an average of $5 \%$. If we assume that only the faintest object of the pair is affected, we infer that $2 \%-3 \%$ of objects may have problematic HST photometry due to the effects of neighbors.

\section{QUALITY AND CONSISTENCY TESTS}

We carried out a number of tests to assess the photometric quality of the catalogs. Here we limit the discussion to internal tests: we ask whether the colors and uncertainties are reasonable and also whether there are offsets between the five fields. In Appendix $\mathrm{C}$ we do external tests, comparing the photometry in our catalogs to other published surveys. In what follows, we assume that all objects defined as point sources (see Section 3.7) are stars, which is likely to be true for the majority of objects. We note that QSOs may be labeled as stars in our catalogs.

\subsection{Colors}

In Figure 16 we compare the WFC3 colors of objects in the five fields. The top panels show the relation between $J_{F 125 W}-$ $H_{F 160 W}$ color and $H_{F 160 W}$ magnitude in each of the fields. The bottom panels show the relation between $H_{F 140 W}-H_{F 160 W}$ color and magnitude. Stars (objects with star_flag $=1$ ) are shown in red. There is scatter in the colors of stars and galaxies, reflecting the fact that not all stars and galaxies have identical $J-H$ colors. In order to assess whether there are offsets between the fields we assume that the median observed $J-H$ colors do not have a strong field dependence. The red and blue lines show the median color in the magnitude range $18<H_{F 160 W}<22$ for stars and galaxies respectively. The median values are listed in the figure.

We find that the median WFC3 colors show very little field dependence. The rms field-to-field variation in the median $J_{F 125 W}-H_{F 160 W}$ color is 0.016 mag for both stars and galaxies. The difference between the highest and lowest field is $0.04 \mathrm{mag}$. The offsets of stars and galaxies are uncorrelated: subtracting the median color of the stars from the median color of the galaxies in each field does not reduce the field-to-field scatter. Taking the average of the median color of stars and the median color of galaxies does reduce the scatter, to an rms of only 0.01 mag and a maximum difference of 0.03 mag between the fields. The $H_{F 140 W}-H_{F 160 W}$ colors show even less variation between fields than the $J_{F 125 W}-H_{F 160 W}$ colors: the rms field-to-field variation in the average color of stars (galaxies) is 0.013 (0.010) mag, and the maximum difference is $0.03 \mathrm{mag}$.

Figure 17 shows color-color relations in wavelength ranges that are particularly useful for separating stars from galaxies. There are subtle differences in the distributions of galaxies, partly due to nonuniformity in the ground-based filters that are used in the five fields. However, stars are well separated from galaxies in each field, particularly in the bottom panels of Figure 17. We infer that the star/galaxy separation is excellent, at least for objects with $K<24.5$.

\subsection{Total Fluxes}

As is usually the case, the colors of objects in the catalogs are determined with higher accuracy than their total fluxes. Colors, and (more generally) the shapes of the SEDs of objects, are measured using carefully matched apertures. However, total fluxes are based on measurements in SExtractor's AUTO aperture, corrected on an object-by-object basis for flux falling outside of this aperture (see Section 3.4). As described in Section 3.6, total fluxes were empirically determined for the $H_{F 160 \mathrm{~W}}$ band only; all other bands were corrected to a total flux using the ratio of total flux to color aperture flux in the $H_{F 160 W}$ band. As a result, the shapes of the SEDs in our catalog are based on psf-matched photometry using a reference aperture of 0.7 , and their normalizations are based on the total $H_{F 160 W}$ flux. We note here that only the HST bands are true 0.7 aperture measurements. The shapes of the SEDs of galaxies with strong color gradients outside of this aperture may have small systematic errors, in particular in the IRAC bands.

We test the accuracy of the total flux measurements in two ways. First, we measure fluxes in large apertures directly from the WFC3 mosaics. Figure 18 shows $F_{\text {ap }} / F_{\text {tot }}$, the ratio of these aperture magnitudes and the total flux as listed in the catalog, as a function of aperture size. Stars are shown as red lines, with median values indicated by open star symbols. The 

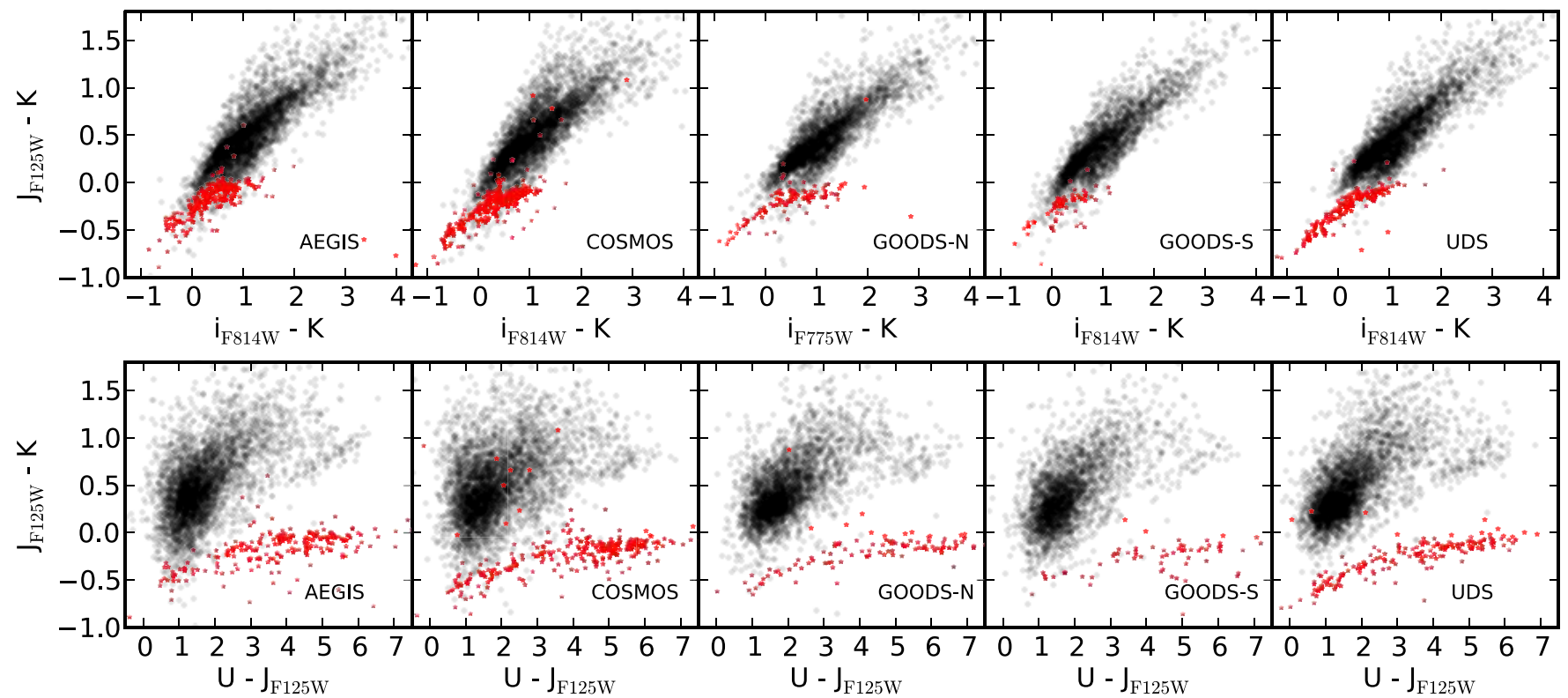

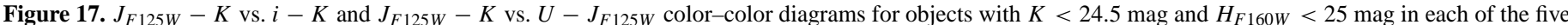

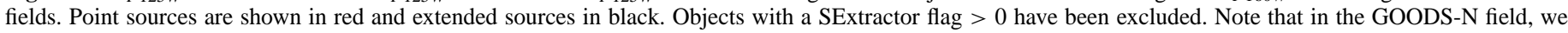

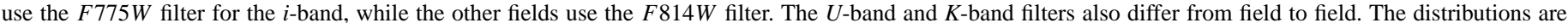
consistent with each other. The stellar locus can be clearly identified in color-color space.

(A color version of this figure is available in the online journal.)

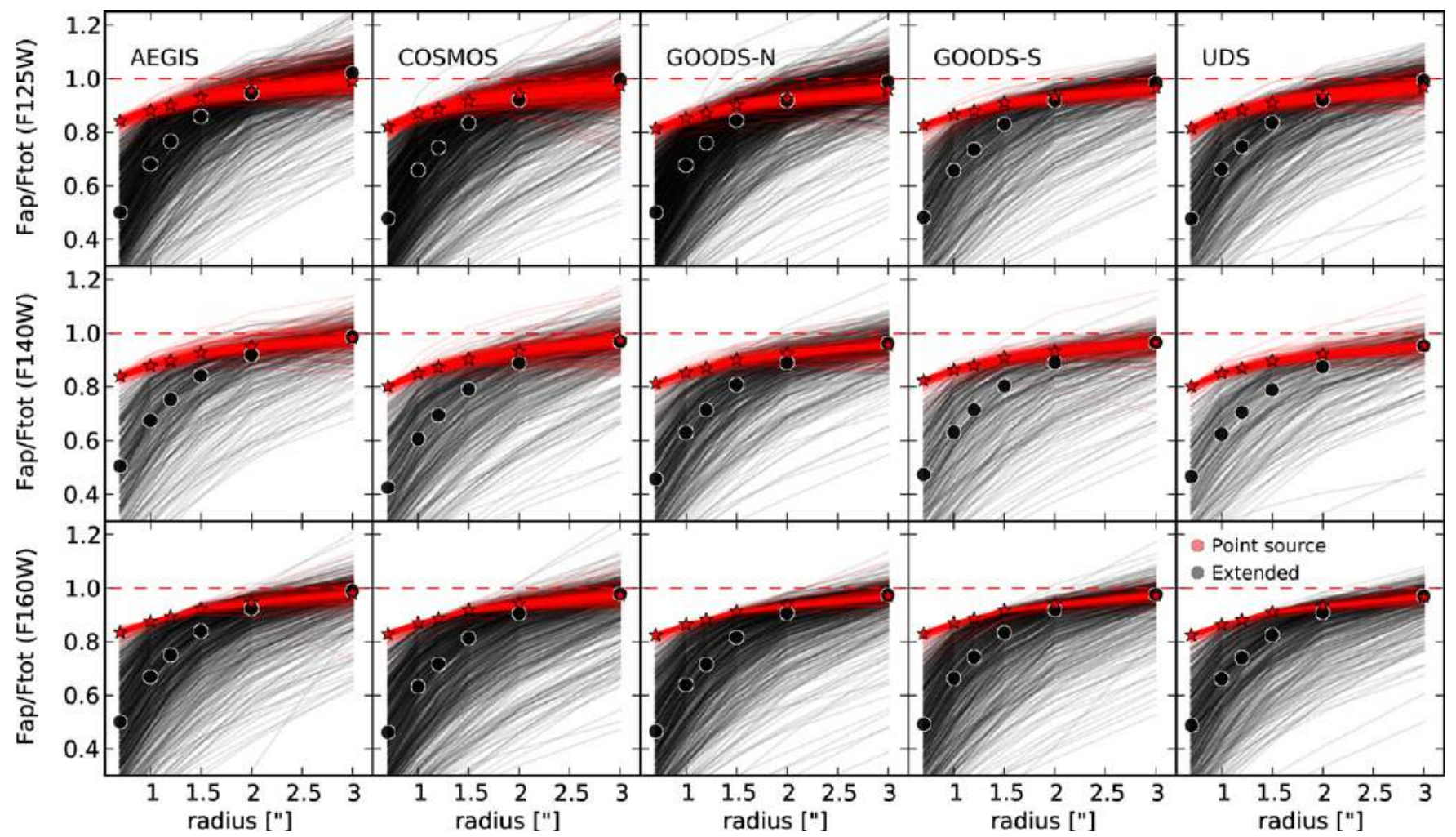

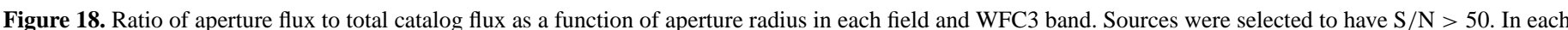

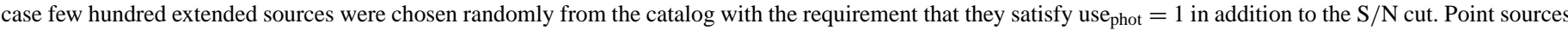

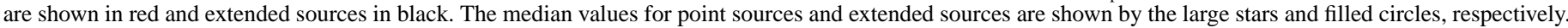

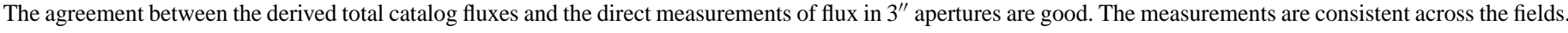
(A color version of this figure is available in the online journal.)

growth curves show little scatter, and reach values that are within 0.05 mag of unity for an aperture radius of $3^{\prime \prime}$. As our correction to total fluxes is partly based on the growth curves of stars this is not surprising; nevertheless, this test empirically demonstrates that stellar photometry is reliable. The grey curves and black points show growth curves of galaxies. There is a large variation in the curves, reflecting the large variation in the apparent sizes of galaxies. Rather remarkably, the median growth curves again reach unity (within $0.05 \mathrm{mag}$ ) at the largest aperture sizes, in all three filters and in all five fields. This implies that our correction 


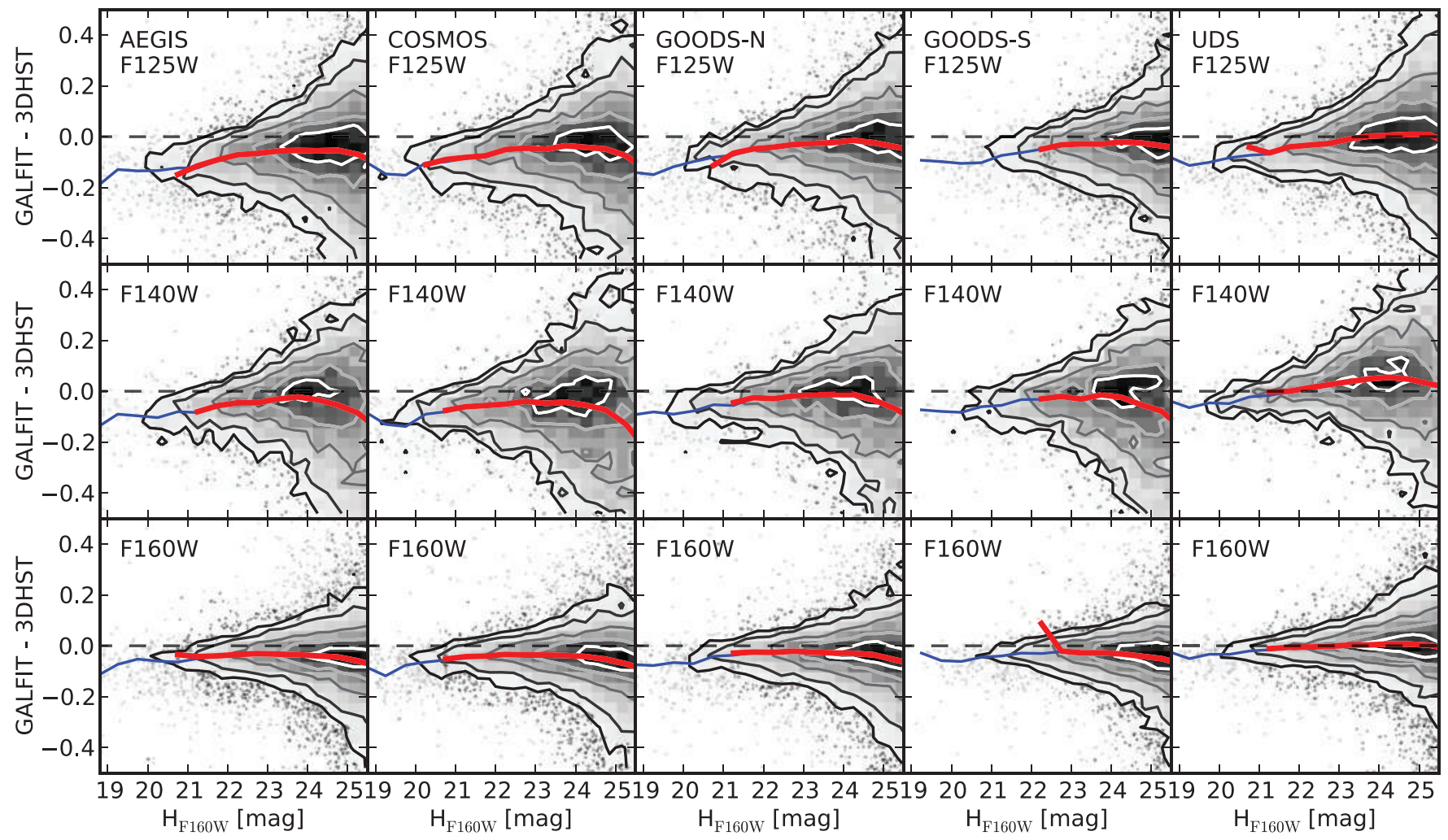

Figure 19. Comparison of the 3D-HST WFC 3 total magnitudes to total magnitudes from morphological fits with GALFIT measured on the same images (see van der Wel et al. 2012). Objects with a good fit from GALFIT (GALFIT flag $=0$ ) are shown dark gray, while objects with a suspicious fit (GALFIT flag $=1$ ) are shown in light gray. Objects with a bad fit from GALFIT (GALFIT flag $>1$ ), a 3D-HST use_phot flag $=0$ or SExtractor flags $\geqslant 2$ have been excluded from the comparison. The running medians for GALFIT flag $=0$ and flag $=1$ objects are shown in red and blue, respectively. The contour levels are 5\%, 10\%, 25\%, 50\% and $75 \%$ of the maximum density.

(A color version of this figure is available in the online journal.)

to total fluxes (and the PSF correction for extended galaxies) is correct to a few percent-in the restricted sense that our catalog values correspond, in the median, to the measured flux in a large aperture.

The second test is similar to the first in the sense that it also compares total fluxes in our catalog to total fluxes that were measured in a different way. The alternative measurement here is not an aperture flux but the total flux as implied by the best-fitting Sérsic (1968) model. We used GALFIT (Peng et al. 2010) to fit these models to galaxies in the catalog, using the same procedures as described in van der Wel et al. (2012). The GALFIT total fluxes are the best-fitting Sérsic models integrated to $r=\infty$, and if galaxies are well-represented by Sérsic models out to large radii these fluxes are "true" total fluxes.

Figure 19 shows the difference between total catalog magnitude and total GALFIT magnitude as a function of total catalog magnitude, in the three WFC3 bands and for all five fields. Over the full magnitude range the median difference is $\leqslant 0.06 \mathrm{mag}$ in each field. The median differences in the range $21<H_{F 160 W}<24$ are very small: $-0.03,-0.04$, $-0.03,-0.03$, and $0.00 \mathrm{mag}$ for $H_{F 160 \mathrm{~W}}$ in AEGIS, COSMOS, GOODS-N, GOODS-S, and UDS respectively. The GALFIT magnitudes are slightly brighter, presumably because the Sérsic profiles take into account that the flux of galaxies exceeds that of point sources outside of the AUTO aperture. The differences are also small in the bluer bands: the median difference for the five fields is -0.04 in $J_{F 125 W}$. This is remarkable given that galaxies have color gradients: we use the $H_{F 160 W}$ total magnitudes to correct all other bands, and as galaxies generally become bluer with increasing radius one might have expected that the $H_{F 160 W}$ correction underestimates the needed correction in bluer bands.

In Figure 20 we compare the magnitude difference in two WFC3 bands ( $F 125 W$ versus $F 160 W$ in the upper panels, $F 140 \mathrm{~W}$ versus $F 160 \mathrm{~W}$ in the lower panels) for galaxies with $21<H_{F 160 W}<24$ mag, SExtractor flags $<2$, use_phot $=1$ and a good GALFIT fit (GALFIT flag $=0$ ). Figure 20 shows that the differences for individual galaxies are correlated, such that a relatively high GALFIT flux in one band also implies a relatively high GALFIT flux in another band. This shows that the observed differences for individual galaxies are not dominated by noise. It also suggests that the differences are caused by the structure of the galaxies and not by color gradients, as color gradients would not lead to correlations between offsets in $H_{F 160 W}$ and offsets in other bands.

\subsection{Error Estimates}

The errors in the photometry were determined by placing "empty apertures" in each of the images, and determining the width of the distribution of flux measurements (see Section 3.4). This method, described in more detail in Labbé et al. (2003), closely approximates the methodology that is used for the actual flux measurements and is insensitive to noise correlations (which affect error estimates based on the observed pixelto-pixel variation, such as the errors that are calculated by SExtractor). As described in Section 3.4, we ensured that the error for each object is adjusted to take into account the photometric weight at its position.

In Figure 21 we show the catalog errors as a function of $H_{F 160 W}$ magnitude in each of the five fields. The top panels 


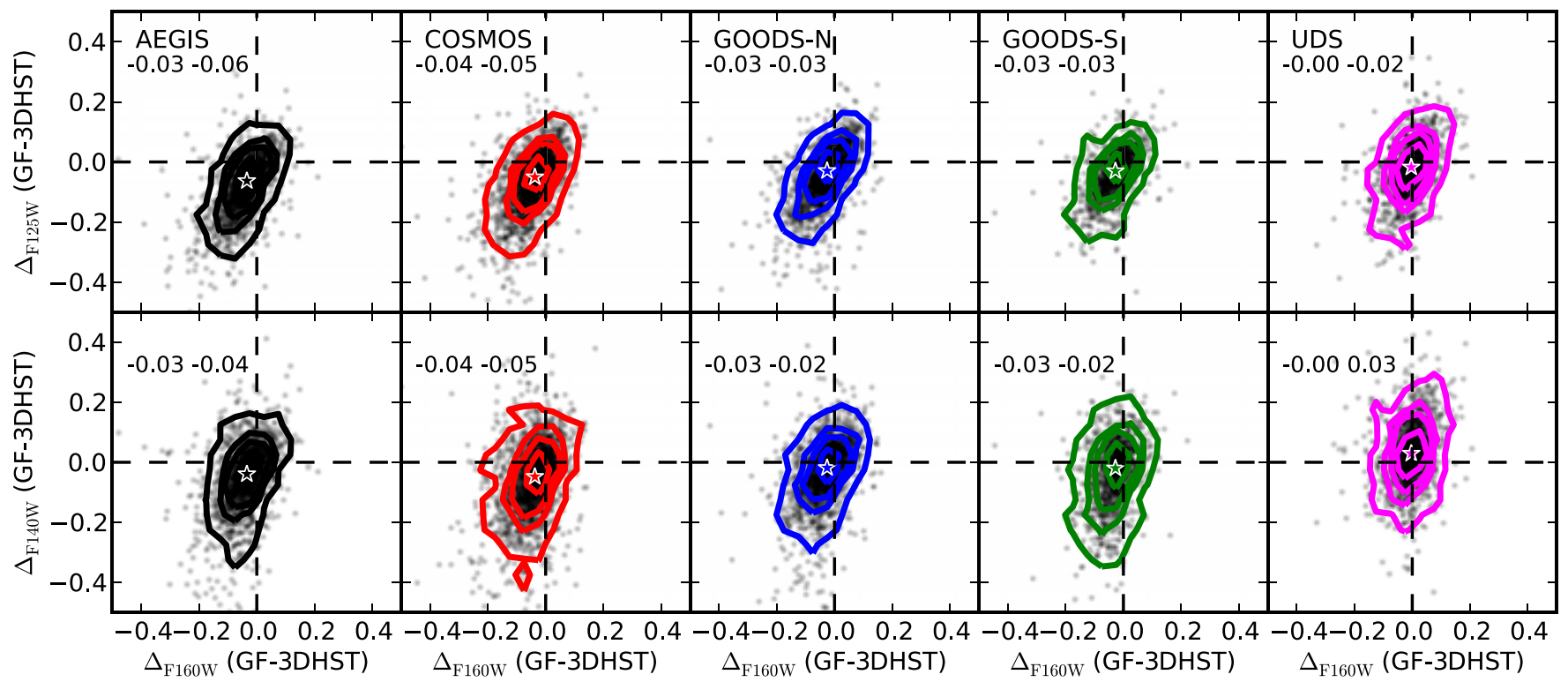

Figure 20. Difference between total magnitudes from GALFIT and 3D-HST in two WFC3 bands for $21<H_{F 160 W}<24$. The upper (lower) panels compare the $J_{\mathrm{F} 125 \mathrm{~W}}\left(H_{\mathrm{F} 140 \mathrm{~W}}\right)$ and the $H_{\mathrm{F} 160 \mathrm{~W}}$ magnitude differences. Only objects with good GALFIT fits (GALFIT flag $\left.=0\right)$ and 3DHST use $\_$phot $=1$ are included. The medians are shown with a star symbol and labelled in the upper left corner of each plot. The contour levels represent $2 \%, 10 \%, 20 \%$ and $50 \%$ of the maximum density. The differences are correlated and smallest in $H_{\mathrm{F} 160 \mathrm{w}}$.

(A color version of this figure is available in the online journal.)

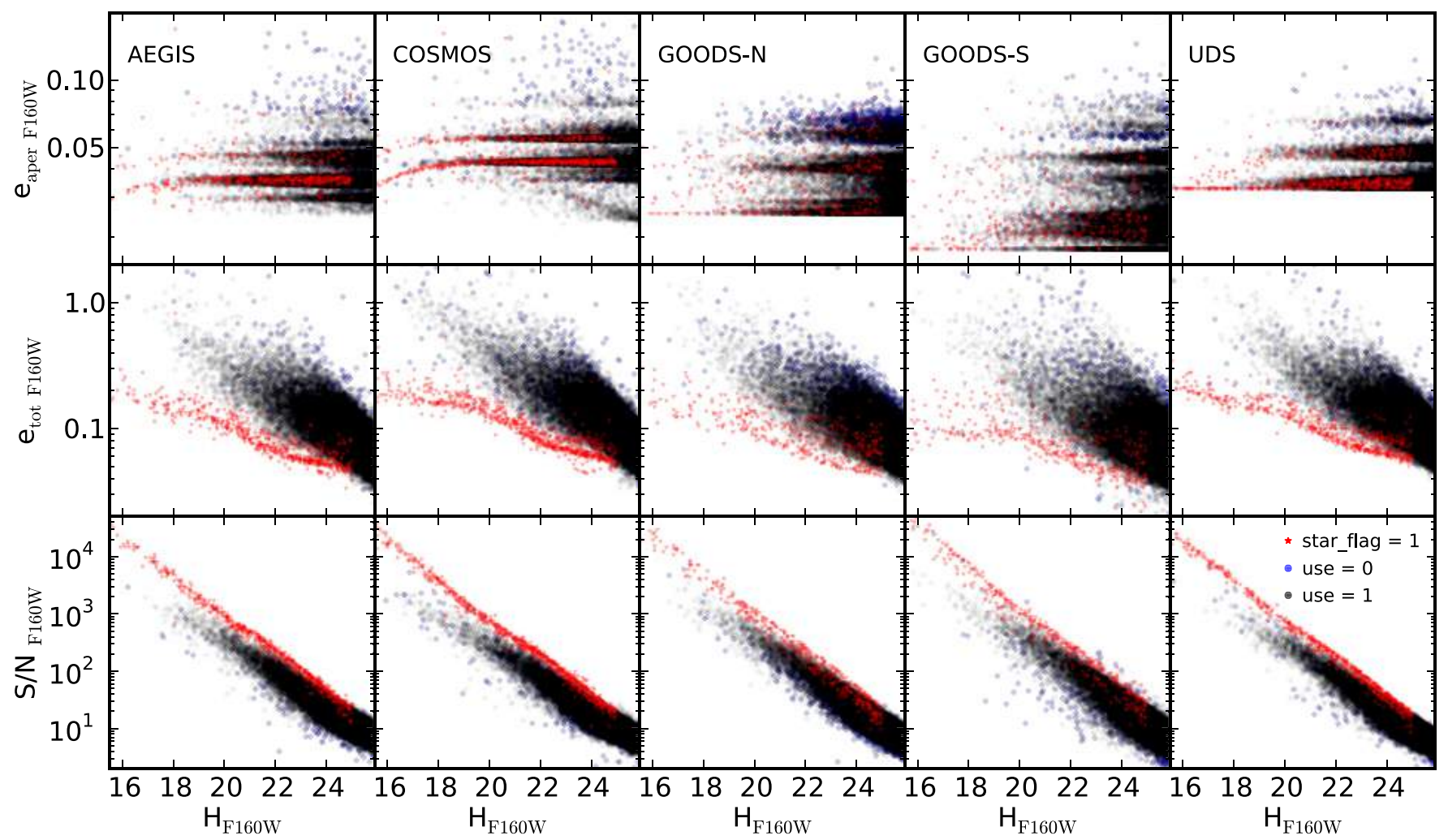

Figure 21. $F 160 \mathrm{~W}$ error distributions in each of the five fields. The units are such that $\mathrm{AB}=25-\log$ (value). Objects with use_phot $=0$ are shown in blue if they are extended or in red if they are point sources (star_flag $=1$ ). Galaxies defined with use_phot $=1$ are shown in black. Upper panels: $F 160 \mathrm{~W}$ errors within an aperture of 0.7 vs. magnitude. The variable depths across each mosaic give rise to the discrete levels. GOODS-S is the deepest image with aperture errors reaching the lowest values, while COSMOS is the shallowest field on average. Middle panels: total $F 160 \mathrm{~W}$ error vs. magnitude, as determined by scaling the noise within empty apertures to the aperture size of the circularized Kron radius of each object and making a correction to total as described in the text. Lowest panels: $F 160 \mathrm{~W}$ (total) signal to noise vs. magnitude. Point sources have the highest $\mathrm{S} / \mathrm{N}$ at a given magnitude, while objects that should be excluded from analysis because they have low weight (and correspondingly large errors) form the lower envelope of the distribution.

(A color version of this figure is available in the online journal.) 


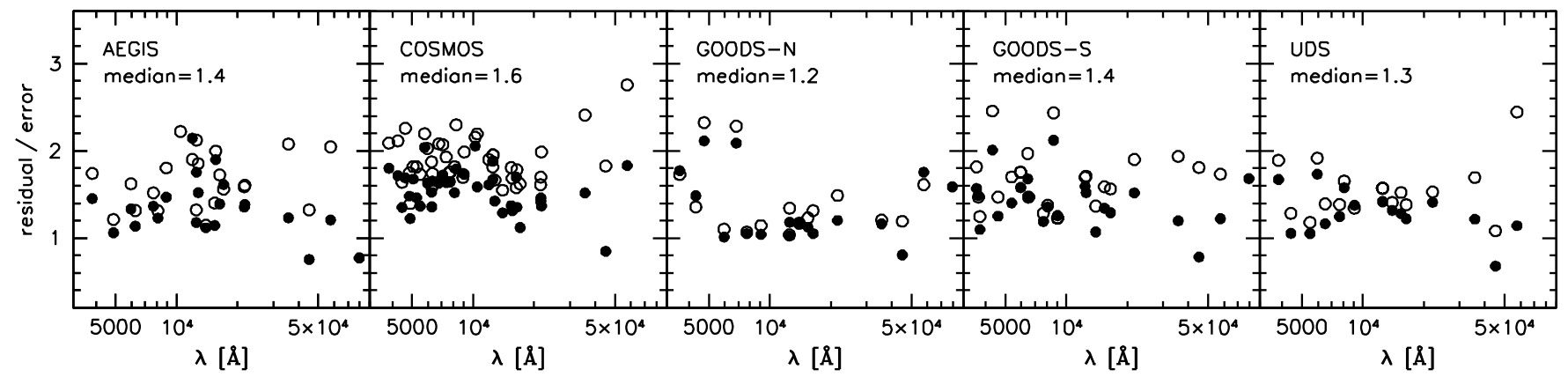

Figure 22. Comparison of the photometric uncertainties to the actual scatter in the data. For each filter in each field, the observed fluxes were compared to the best-fitting EAZY model. The panels show the average residual from the fit divided by the expected residual based on the photometric uncertainty. Open symbols show the catalog values, solid symbols show the catalog values with the EAZY template error function added in quadrature. If the uncertainties are accurate the average should be 1 . The uncertainties are slightly overestimated in most filters, but only by $20 \%-50 \%$.
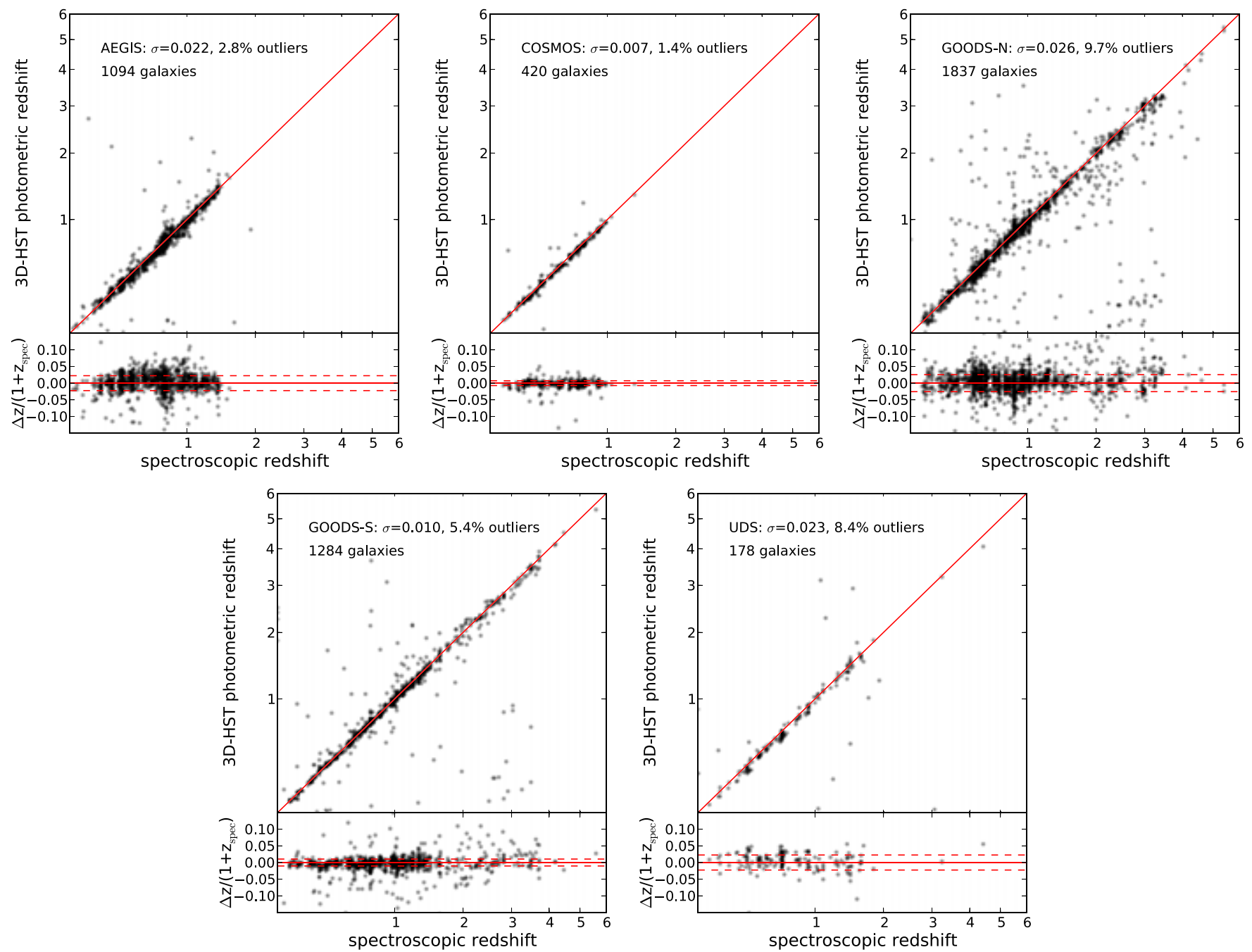

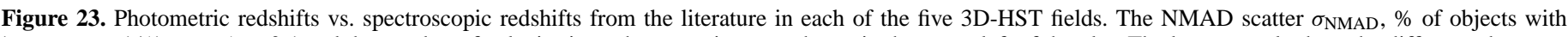

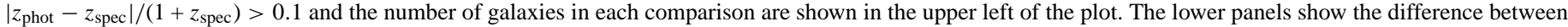
the photometric and spectroscopic redshifts over $1+z_{\text {spec }}$. The red dashed lines indicate $\pm \sigma_{\mathrm{NMAD}}$ in each case.

(A color version of this figure is available in the online journal.)

show the errors in our standard photometric aperture of 0.7 . The scatter in the error at fixed magnitude is caused by the variation in the depth of the $H_{F 160 W}$ mosaics. The stripes reflect the fact that the weights, and hence the errors, largely reflect the number of exposures that went into a particular position in the mosaic, and this number is an integer. Stars (red) fall in the same bands as galaxies, as their aperture fluxes are measured in the same $0 \prime^{\prime} 7$ aperture. The distributions are not identical in each field, as the depths are not identical. The error distribution in GOODS-S extends to very small errors, reflecting the great depth of the Ultra Deep Field data.

The middle panels show the errors in the "total" aperture. These errors are determined from the empty aperture errors using the power-law fit at the number of pixels in the circularized 


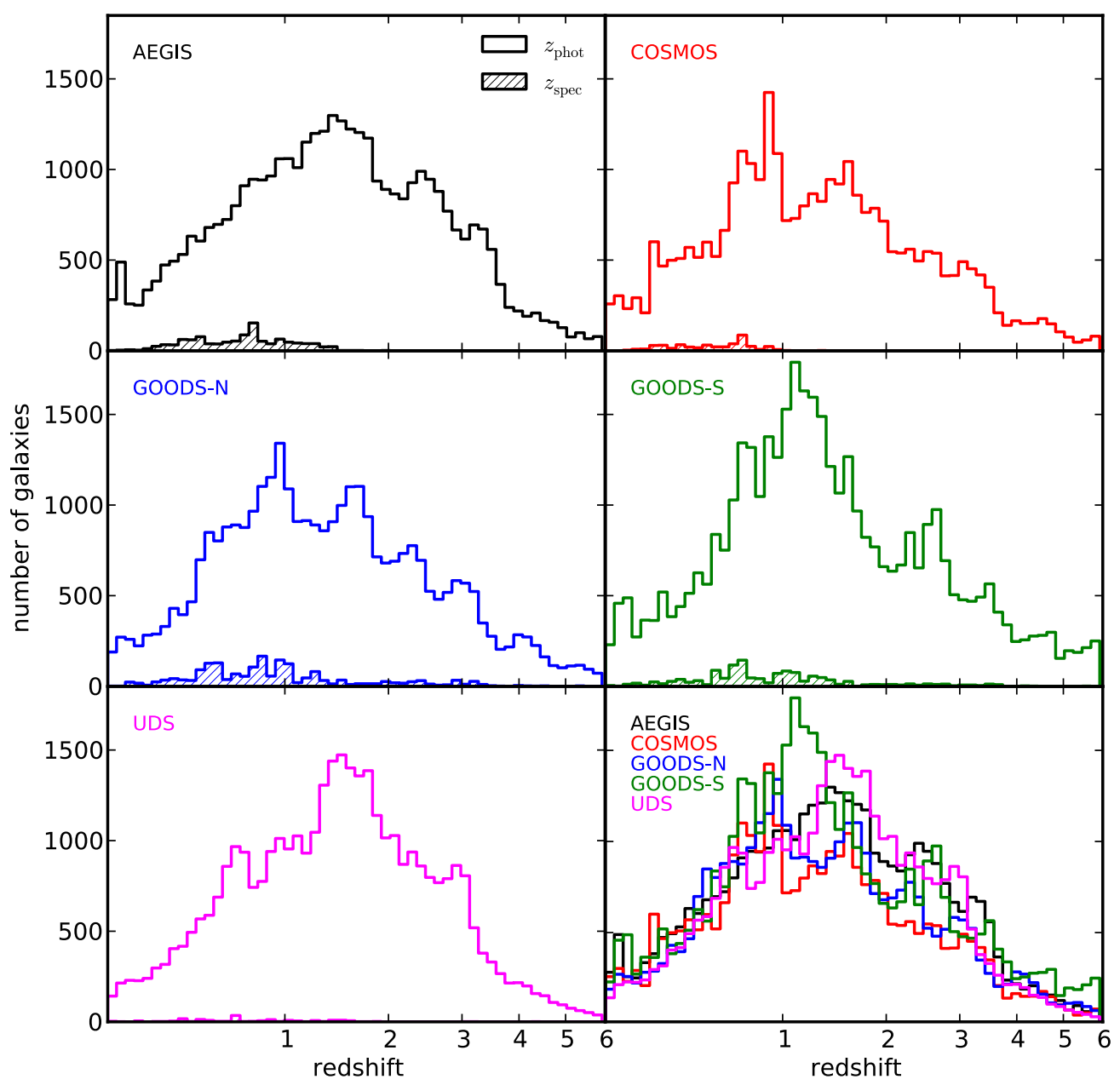

Figure 24. Redshift distribution in each of the five fields. The distribution of photometric and spectroscopic redshifts are shown as the open and hatched histograms, respectively. The final panel shows the distribution of photometric redshifts for all five fields, for comparison.

(A color version of this figure is available in the online journal.)

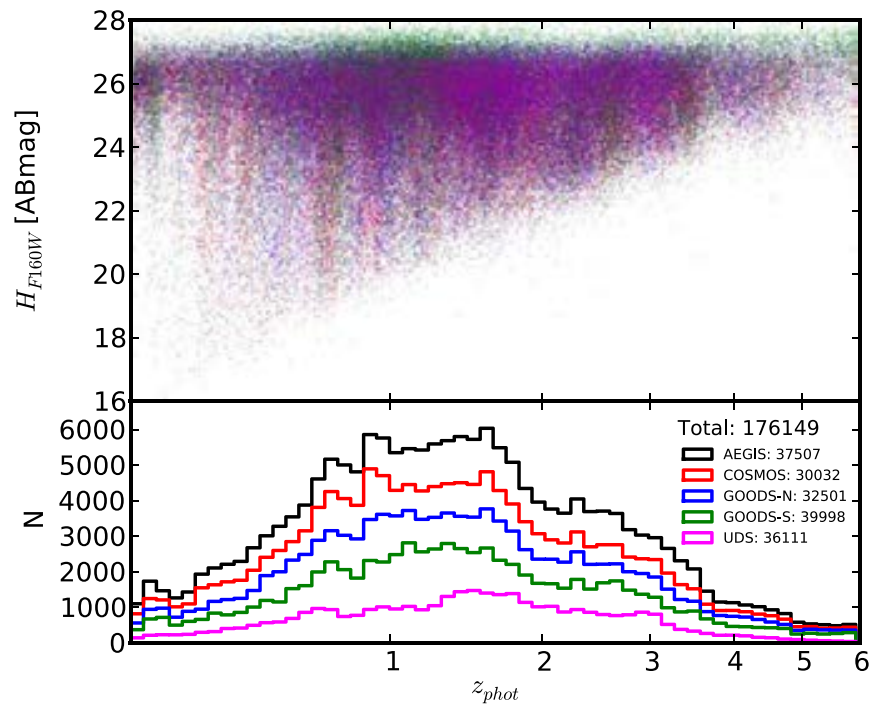

Figure 25. Distribution of $H_{F 160 W}$ apparent magnitudes with photometric redshift (z_peak), color-coded by field. The lower panel shows the number of galaxies as a function of $z_{-}$peak broken down into the contribution from each field. The histograms are successively added, with the black histogram giving the total distribution of all galaxies.

(A color version of this figure is available in the online journal.)
Kron aperture (see Figure 12 and Section 3.4). The stripes are blurred in these panels, as the scatter in the error at fixed magnitude is now dominated by the variation in the Kron aperture size at fixed magnitude. The scatter in the Kron aperture size reflects, in turn, the scatter in the sizes of galaxies at fixed magnitude. Stars are now clearly offset from galaxies: as the total flux of stars is measured in a smaller aperture than the total flux of extended sources the errors in their total fluxes are smaller. The scatter in the errors of stars is caused by the variation in weight: this also explains why some galaxies have smaller errors than some stars, particularly in GOODS-S (which has the largest depth variation of any of the fields).

In the bottom panels the $\mathrm{S} / \mathrm{N}$ is plotted as a function of magnitude. The $\mathrm{S} / \mathrm{N}$ was calculated by dividing the total $H_{F 160 \mathrm{~W}}$ flux by the error in the total aperture. The relation of the $\mathrm{S} / \mathrm{N}$ of stars with magnitude shows very little scatter, reflecting the small scatter in the errors of stars in the middle panels. Galaxies show a large scatter. We note that the depths we, and others, quote for our data reflects the depth for point sources (red points). The errors in the total magnitudes of galaxies are typically much larger, and this should be taken into account when assessing the quoted depth of the $H_{F 160 W}$ images (see Section 3.10). By contrast, the errors in the colors of galaxies, and the errors in the catalog for all bands except $H_{F 160 \mathrm{~W}}$, are independent of the size of the object as they are based on the error in the 0.7 aperture. 


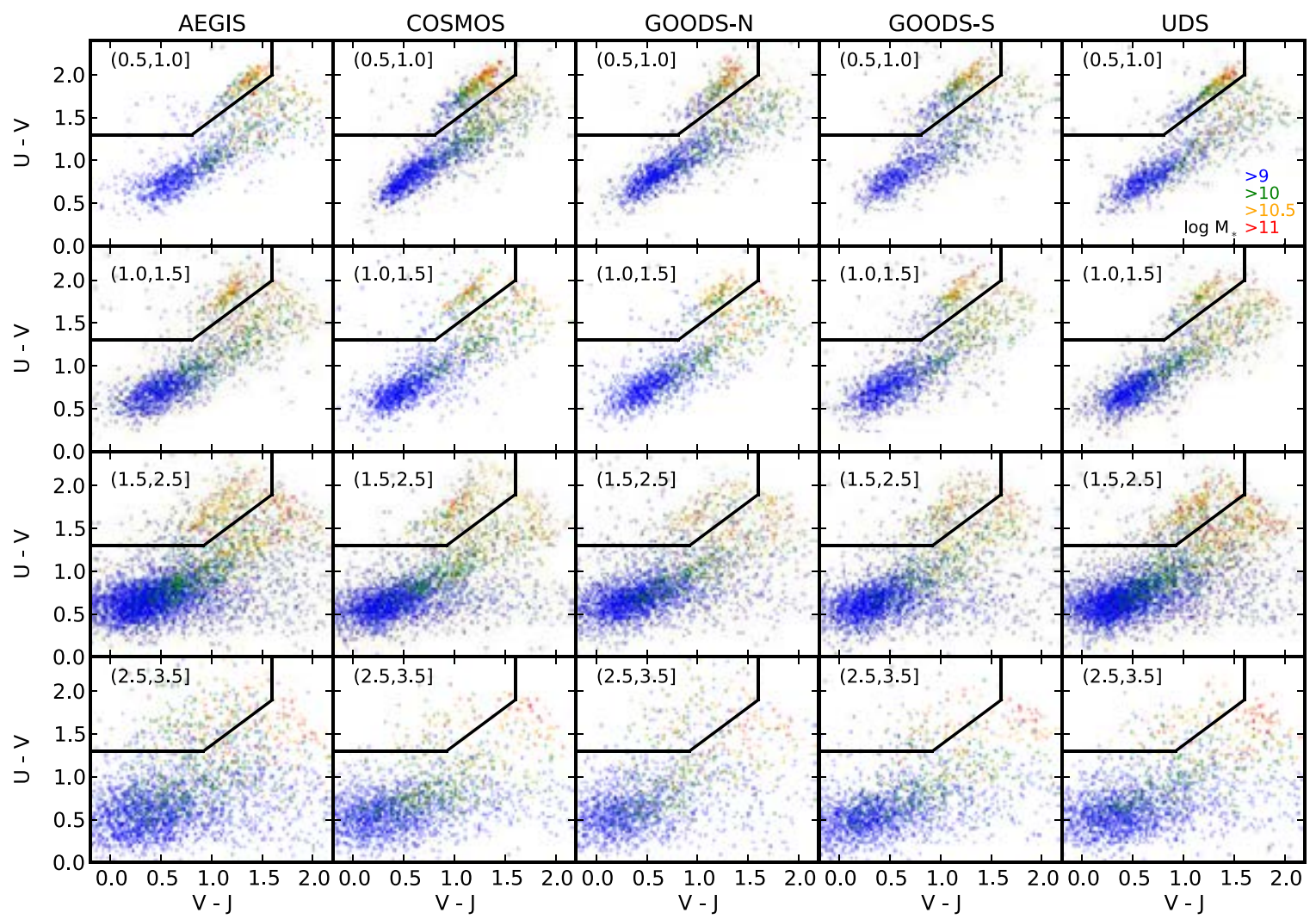

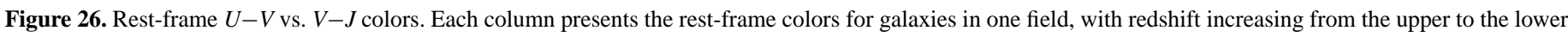

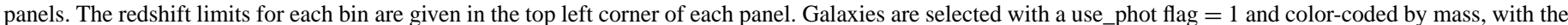

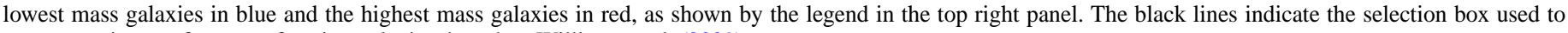
separate quiescent from star-forming galaxies, based on Williams et al. (2009).

(A color version of this figure is available in the online journal.)

The previous tests show that the errors behave as expected, but they do not demonstrate that they are reasonably close to the true errors in the measurements. We tested this in the following way. For each photometric band in each field we selected all objects with a $\mathrm{S} / \mathrm{N}$ ratio of 15 in that band. Next, we subtracted the bestfitting EAZY template (see Section 5.2) from the observed flux, and multiplied the residual by 15 . Finally, we determined the biweight scatter in these distributions. If the errors are correct and the EAZY templates are perfect then we expect to find that these distributions have a scatter of exactly 1 . The results are shown in Figure 22, for the catalog values (open symbols) and the catalog values modified by the EAZY template error function (solid symbols). We find that the scatter in these normalized residuals is close to 1 for nearly all filters in all fields. The median deviations for the fields are between 1.2 and 1.5, implying that the errors are typically underestimated by $20 \%-40 \%$. This is a relatively small effect given that the observed residuals include all possible sources of error, including residual template mismatch.

\section{REDSHIFTS, REST-FRAME COLORS, AND STELLAR POPULATION PARAMETERS}

We used the photometric catalogs to derive photometric redshifts, rest-frame colors, and stellar population parameters of the galaxies in the five fields. As is well known, these derived parameters depend on the methodology that is used to derive them, and on model assumptions (see, e.g., Brammer et al. 2008; Kriek et al. 2009). A "default" set of parameters, described below, is provided with our photometric catalogs from the 3D-HST release pages. We expect to release future updates to these catalogs of derived parameters, in particular versions in which the grism spectroscopic data are used to improve the redshift measurements.

\subsection{Spectroscopic Redshifts}

As a first step we searched the literature and other sources to find (ground-based) spectroscopic redshifts of objects in the five fields. These redshifts are used to assess the quality of photometric redshifts in Section 5.2, at least for objects that are relatively bright at optical wavelengths. Furthermore, when determining rest-frame colors and stellar population parameters we always use this spectroscopic redshift if it is available; otherwise we use the photometric redshift. The spectroscopic redshifts in our catalogs are obtained by cross-matching the positions of objects within 0 .'.5 to a number of publicly available catalogs.

In the AEGIS field there are 1139 spectroscopic redshifts obtained by matching to the DEEP $2+3$ catalogs (Davis et al. 2003; Newman et al. 2013; Cooper et al. 2012). Only objects with the highest quality flags (quality flag $=4$ ) are included in the catalog. There are 1094 galaxies with use_phot $=1$ and a spectroscopic redshift.

For the COSMOS field, we match to the zCOSMOS catalogs (Lilly et al. 2007), finding 383 spectroscopic redshifts. We additionally include 72 spectroscopic redshifts determined from MMT/Hectospec data (Fabricant et al. 2005; Mink et al. 


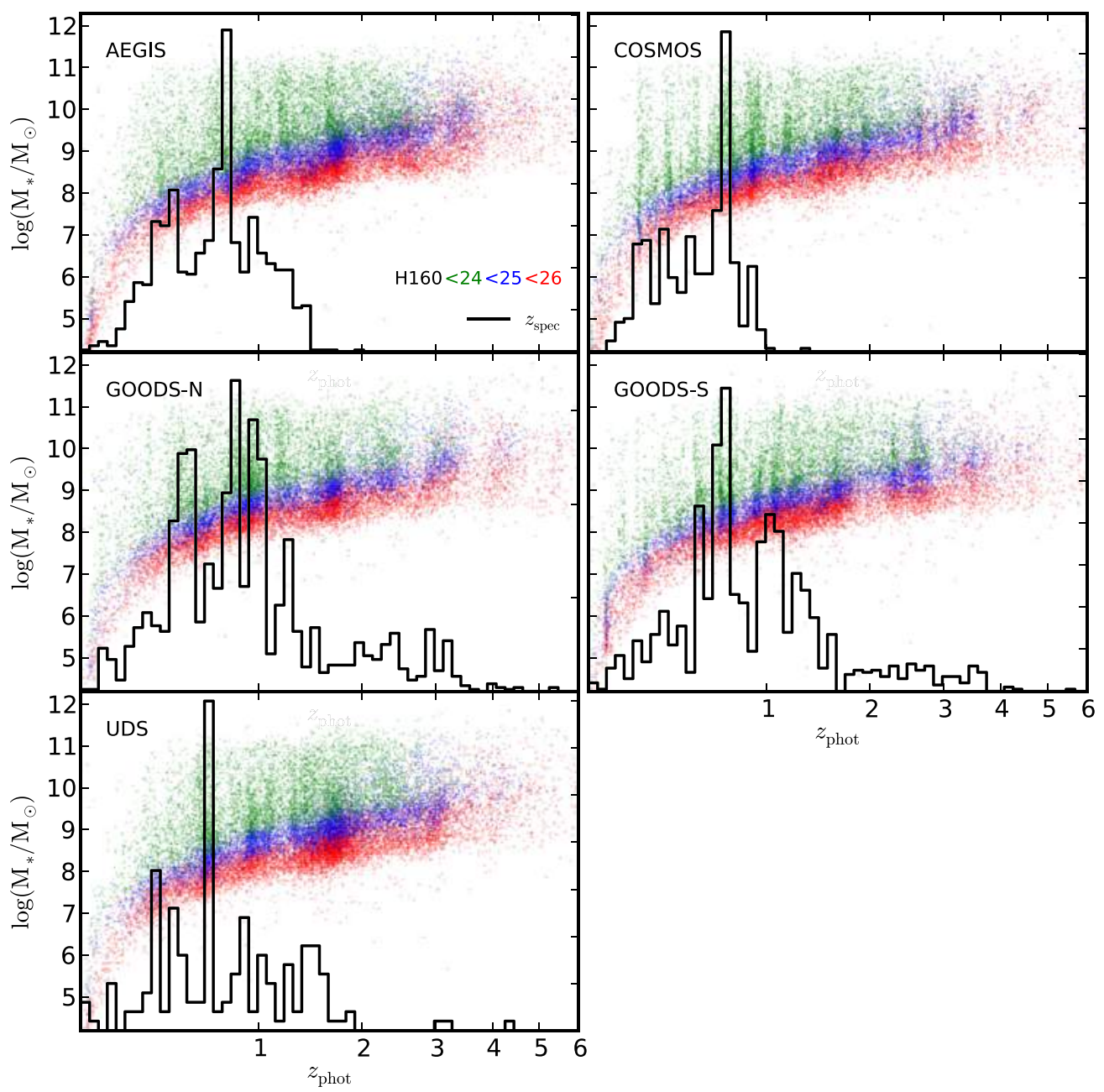

Figure 27. Stellar mass vs. photometric redshift. The points are color-coded by magnitude such that galaxies with $H_{F 160 W}<24$ are in green, $24 \leqslant H_{F 160 W}<25$ in blue and $25 \leqslant H_{F 160 W}<26$ in red. The gray histogram shows the distribution of spectroscopic redshifts from the literature, arbitrarily scaled. Many of the over densities in photometric redshift correspond to peaks in the spectroscopic redshift distribution.

(A color version of this figure is available in the online journal.)
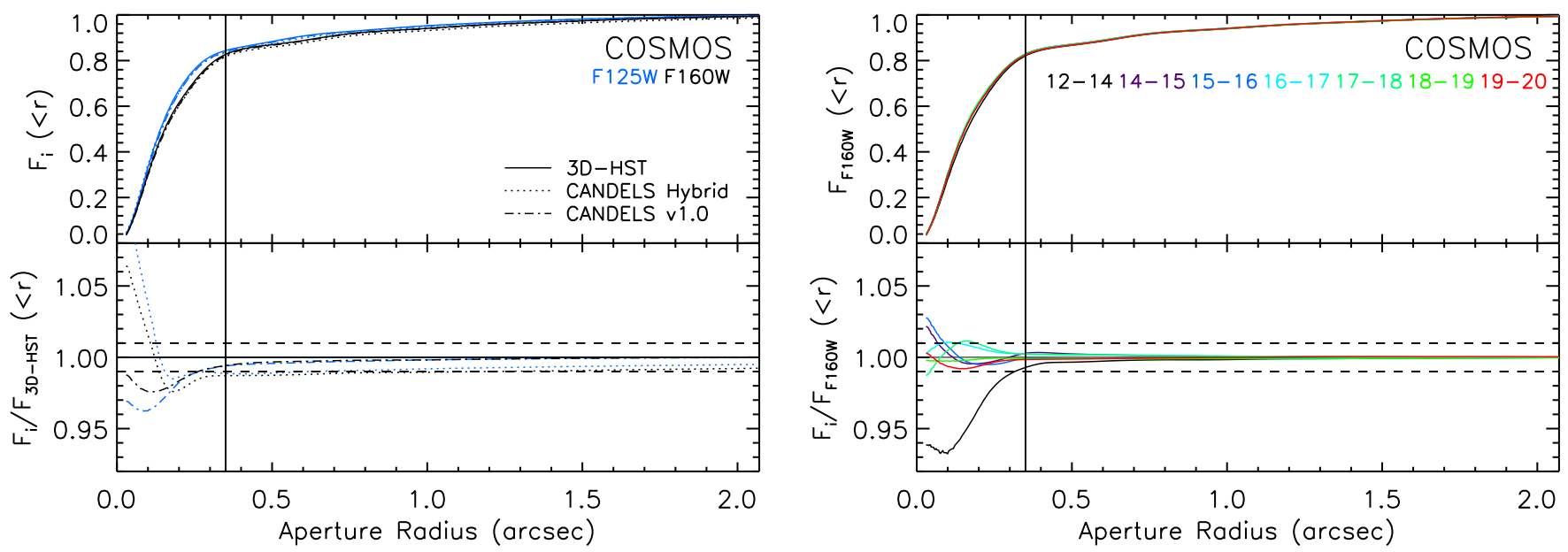

Figure 28. Left panels: a comparison of the $F 160 \mathrm{~W}$ (black lines) and $F 125 \mathrm{~W}$ (blue lines) growth curves from three PSFs for the COSMOS field. The solid lines show the 3D-HST PSFs used in this analysis, the dash-dotted line a PSF made in the same way using the CANDELS v1.0 mosaic, and the dotted line a "hybrid" PSF used for the GALFIT morphological measurements (van der Wel et al. 2012). The hybrid PSF combines an artificial PSF created with Tiny Tim in the inner region with an empirical PSF from a stack of stars in the outer regions. Right panels: A comparison of the growth curves from PSFs created in the COSMOS field using stars in narrow magnitude bins (color-coded as shown in the legend) rather than the full magnitude range. There are very small differences between the PSFs. Saturation affects only the brightest stars, leading to a larger difference in the PSF of stars with $12<H_{160 \mathrm{~W}}<14$. At the aperture used for photometry ( 0 '!35) the differences between the PSFs in all the magnitude bins are smaller than $1 \%$.

(A color version of this figure is available in the online journal.) 

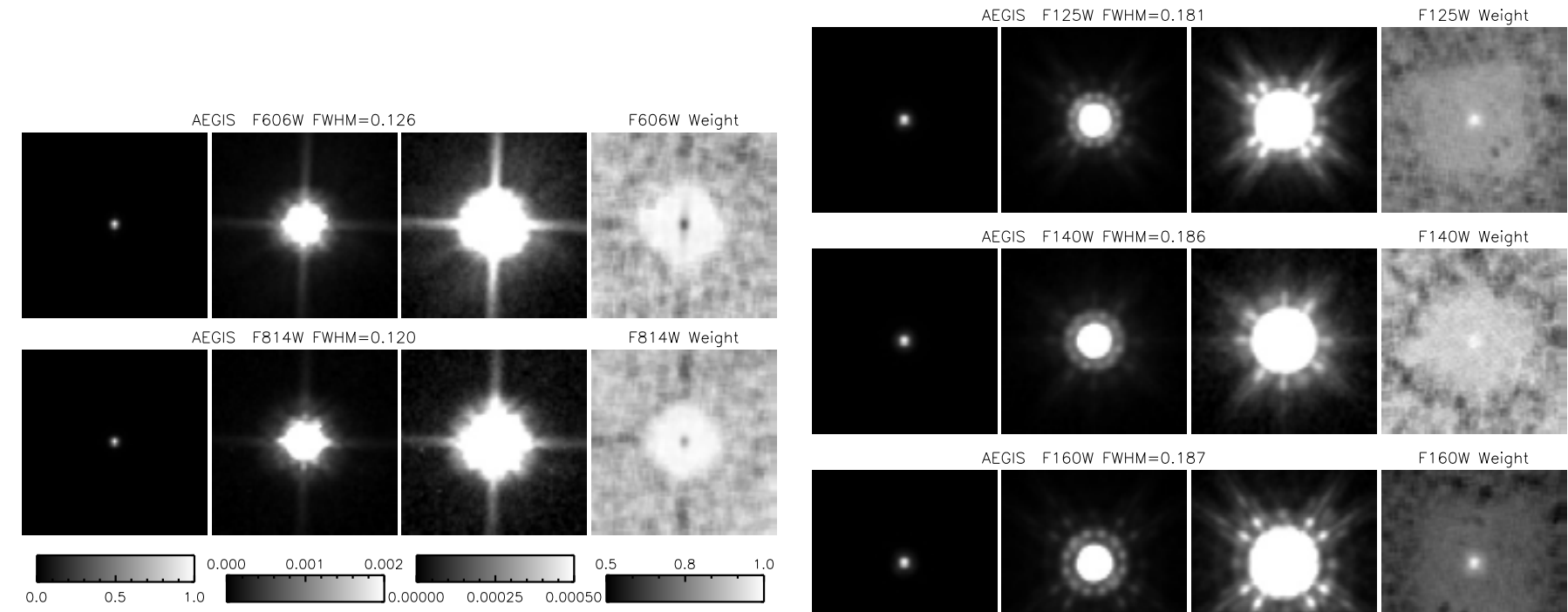

F140W Weight
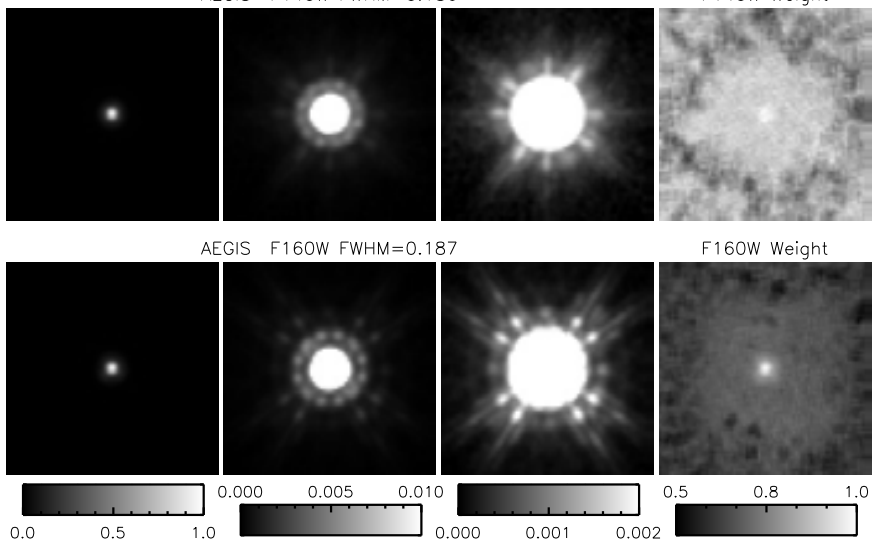

F160w Weight

0.5
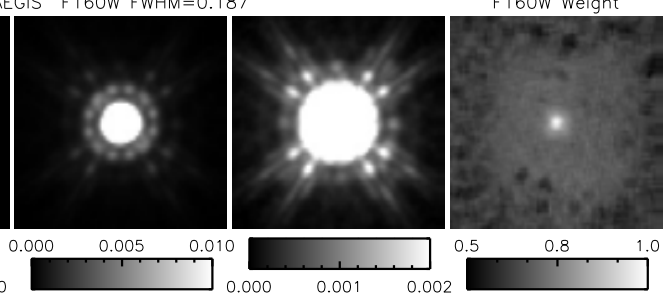

Figure 29. Point-spread functions (PSFs) for the ACS $F 606 W$ and $F 814 W$ bands (left 4 panels) and the WFC $3125 W, F 140 W$, and $F 160 W$ bands (right 4 panels) in the AEGIS field. The construction of the PSFs is described in Section 3.3. Each image is $69 \times 69$ pixels or 4 "' $14 \times 44^{\prime \prime} 14$, i.e., it traces the PSF out to just over 2" radius. For each filter we show three stretch levels (panels 1-3 and 5-7) to expose the structure of the PSF: the core, the first Airy ring and the diffraction spikes. The images are normalized to a maximum value of one. The grayscale bars show the stretch for each panel. These are slightly different for ACS and WFC 3 as a result of the different FWHMs (listed above the images). We also show the combined weight images for each PSF. The weight is largest in the center and lower at larger radii due to masking of neighboring objects. The ACS PSFs have lower weights in the central pixels because of cosmic ray rejection flagging the centers of stars.
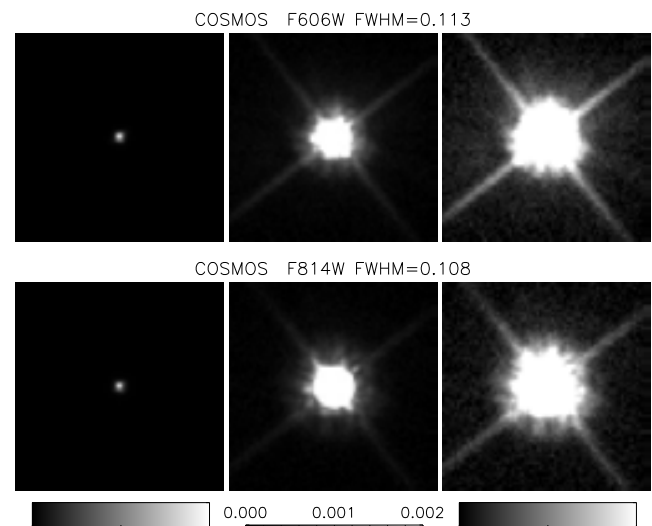

COSMOS F814W FWHM=0.108
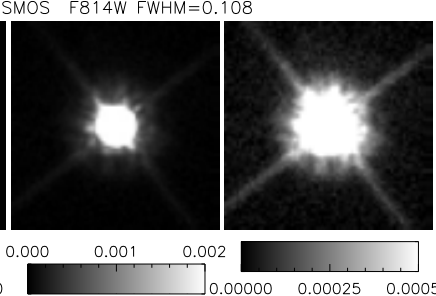

F606W Weight

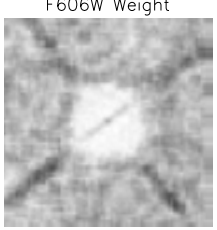

F814W Weight

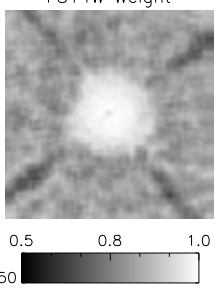

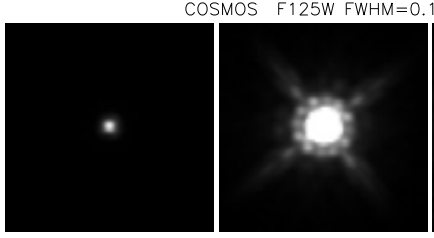

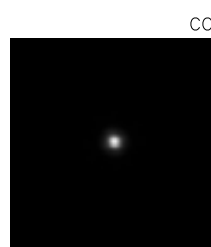

COSMOS F140W FWHM $=0.18$
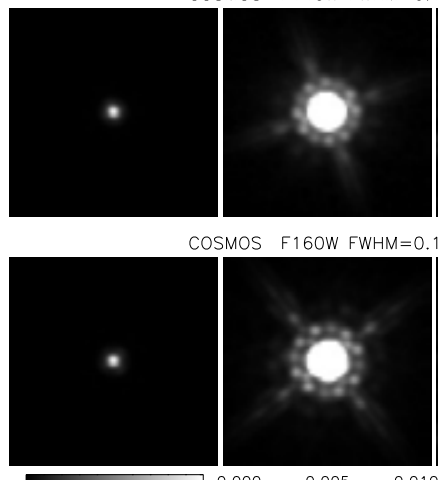

COSMOS F160W FWHM $=0.18$

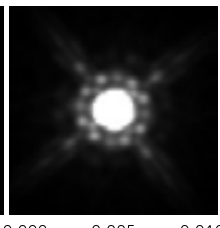

0.005

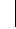

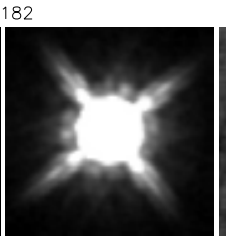
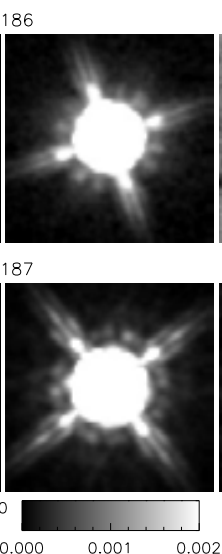

F125W Weight

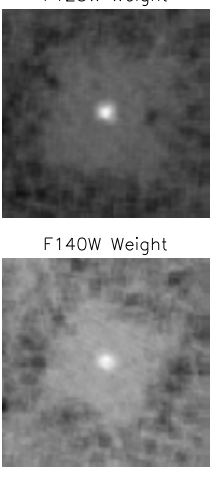

F160w Weight

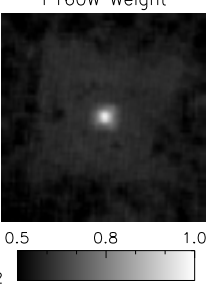

Figure 30. Same as Figure 29 for the ACS and WFC3 PSFs in the COSMOS field.

2007), ${ }^{32}$ bringing the total to 455 spectroscopic redshifts. Here too, we keep only redshifts with an "excellent" quality flag. There are 420 galaxies with use_phot $=1$ and a spectroscopic redshift.

There are 2081 spectroscopic redshifts included in the GOODS-N catalog. These were obtained by matching to the MODS catalog (Kajisawa et al. 2011). The MODS redshifts are compiled from Yoshikawa et al. (2010), Barger et al. (2008), Reddy et al. (2006), Treu et al. (2005), Wirth et al. (2004), Cowie et al. (2004), Cohen (2001), Cohen et al. (2000), Dawson

32 The MMT/Hectospec data include about $8 \mathrm{hr}$ in total, observed on 2011 November 23, 2013 February 2, 2013 March 31 and 2013 April 1 by M. Kriek. et al. (2001). No quality flags were provided, so there is a mix of reliable and less reliable redshifts in this field. There are 1837 galaxies with use_phot $=1$ and a spectroscopic redshift.

In GOODS-S we find 2228 objects match to objects with spectroscopic redshifts in the FIREWORKS catalog (Wuyts et al. 2008). We include the redshifts for the 1445 objects with a FIREWORKS quality flag of 1.0 in the catalog. There are 1284 galaxies with use_phot $=1$ and a spectroscopic redshift.

There are 238 spectroscopic redshifts in the UDS catalog, 182 of which were obtained by matching to the compilation provided on the UDS Nottingham Web page. ${ }^{33}$ The redshifts

\footnotetext{
33 http://www.nottingham.ac.uk/ ppzoa/UDS_redshifts_18Oct2010.fits
} 


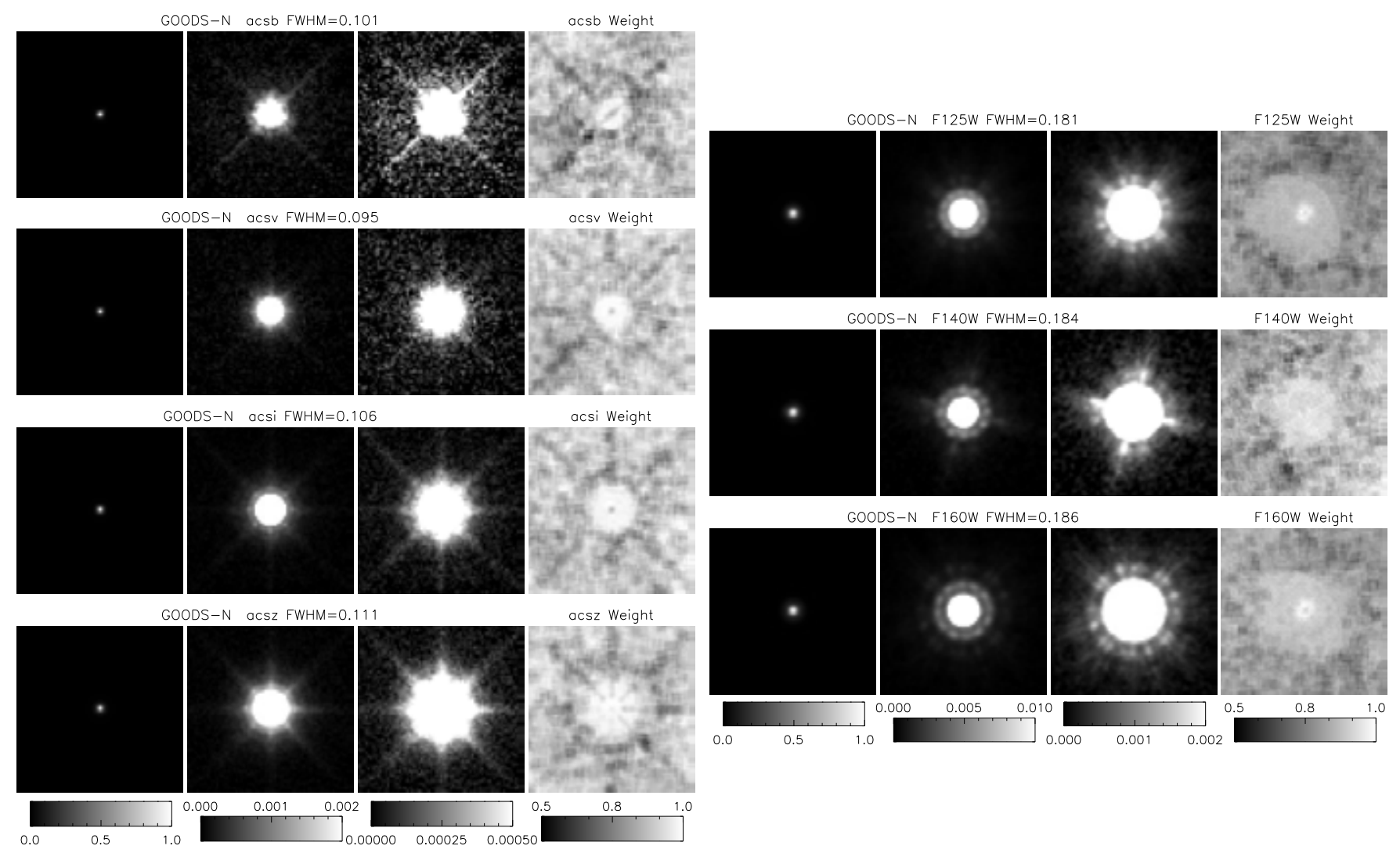

Figure 31. Same as Figure 29 for the ACS and WFC3 PSFs in the GOODS-N field.

are from a variety of sources, with some unpublished at the time the compilation was made (Yamada et al. 2005; Simpson et al. 2006; Geach et al. 2007; van Breukelen et al. 2007; Ouchi et al. 2008; Smail et al. 2008; Ono et al. 2010; Simpson et al. 2012; M. Akiyama et al., in preparation). Redshifts with quality flags A (based on multiple reliable features), B (one reliable feature) or $\mathrm{Z}$ (flag not provided) are included in our catalog. Redshifts with quality flag C (one dubious feature) are not included. We also include 37 spectroscopic redshifts from IMACS/Magellan (Papovich et al. 2010, and I. Momcheva, private communication), 18 redshifts from Bezanson et al. (2013) and 1 redshift from van de Sande et al. (2013). There are 178 galaxies with use_phot $=1$ and a spectroscopic redshift.

\subsection{Photometric Redshifts and Zero Point Corrections}

We determine photometric redshifts by fitting the SED of each object with a linear combination of seven galaxy templates, using the EAZY code (Brammer et al. 2008). ${ }^{34}$ The template set is based on the default set described in Brammer et al. (2008). It contains five templates derived from a library of PÉGASE stellar population synthesis models (Fioc \& Rocca-Volmerange 1997), a young, dusty template and an old, red galaxy template, as described in Whitaker et al. (2011). The old, red galaxy template is derived from the Maraston (2005) stellar population synthesis models with an age of 12.6 Gyr, a Kroupa initial mass function (IMF) and solar metallicity. We use the default template error function scaled by a factor of 0.5 , which helps to account for systematic wavelength-dependent uncertainties in the templates, and a redshift prior based on the $K$-band apparent magnitudes.

\footnotetext{
34 https://code.google.com/p/eazy-photoz/
}

We also modify both the templates and the input photometry in the fitting procedure: the templates are corrected for subtle differences between the observed SEDs of galaxies and the best-fitting templates, and the photometry is corrected for empirically-determined zero point errors. The methodology is discussed in the Appendix. The zero point offsets for each field and band are provided in Table 11. The listed zero points have been applied to the catalogs and the corrected photometry used for the redshift fitting and stellar population parameters we present in the following sections. In what follows, galaxies are selected with a use_phot flag of 1 and we use the spectroscopic redshift, where available, or the peak of the photometric redshift distribution (EAZY's z_peak) as the galaxy redshift, unless specified otherwise.

Figure 23 compares the photometric redshifts to spectroscopic redshifts from the literature. The number and quality of the spectroscopic redshifts in each field are heterogeneous as they are compiled from a number of different sources, as described in Section 5.1. In general we find excellent agreement with a normalized median absolute deviation $\sigma_{\mathrm{NMAD}}=1.48 \times$ $\mathrm{MAD} /(1+\mathrm{z})$ of $<2.7 \%$ in all fields. The improvement in photometric redshifts that arises from including medium band data can be clearly seen by the reduction in scatter in the COSMOS and GOODS-S fields, reaching $\sigma_{\mathrm{NMAD}}=0.007$ and $\sigma_{\mathrm{NMAD}}=0.01$, respectively. Among objects with spectroscopic redshifts, there are few catastrophic failures: $3 \%, 1 \%, 10 \%, 5 \%$, and $8 \%$ for AEGIS, COSMOS, GOODS-N, GOODS-S, and the UDS, respectively, where we define a catastrophic outlier as one with $\left|z_{\text {phot }}-z_{\text {spec }}\right| /\left(1+z_{\text {spec }}\right)>0.1$. We note that X-ray sources have not been excluded from these comparisons. In GOODS-S, 87 objects from the X-ray selected catalog of Szokoly et al. (2004) are included. Removing these sources reduces the outlier fraction and scatter marginally. Ten objects from the XMM catalog 


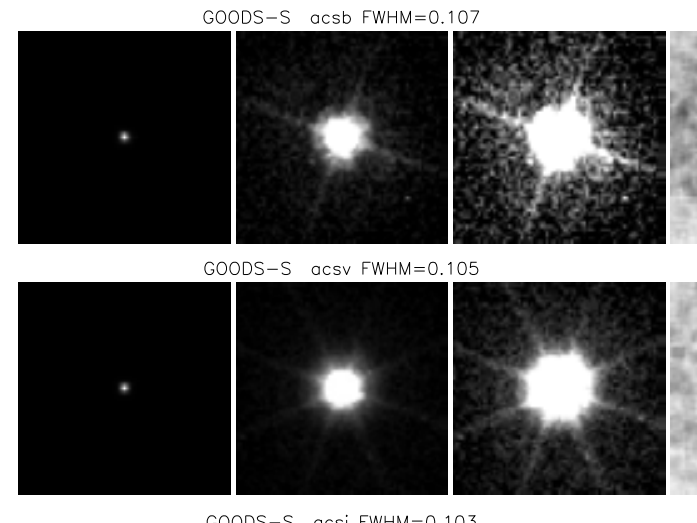

acsb Weight

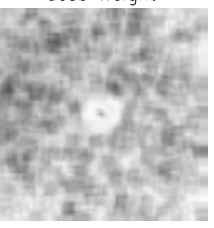

acsv Weight

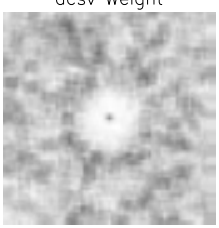

acsi Weight

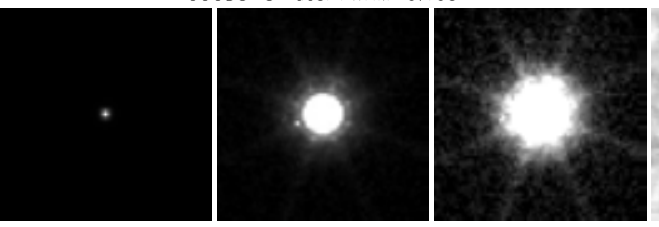

GOODS $-S$ acsz FWHM $=0.110$

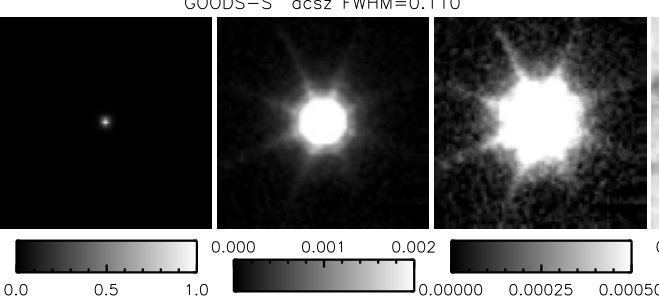

acsz Weight

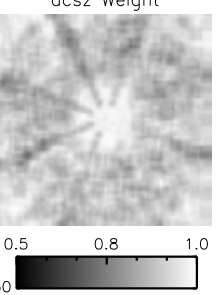

candels_f606w Weight
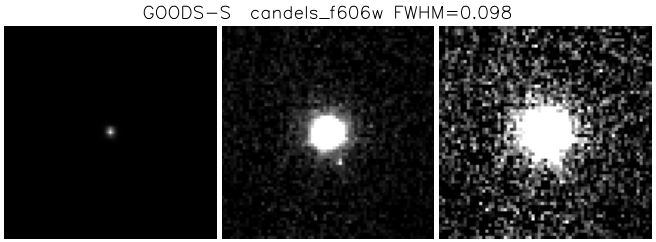

GOODS $-S$ candels_f 814 w FWHM $=0.102$
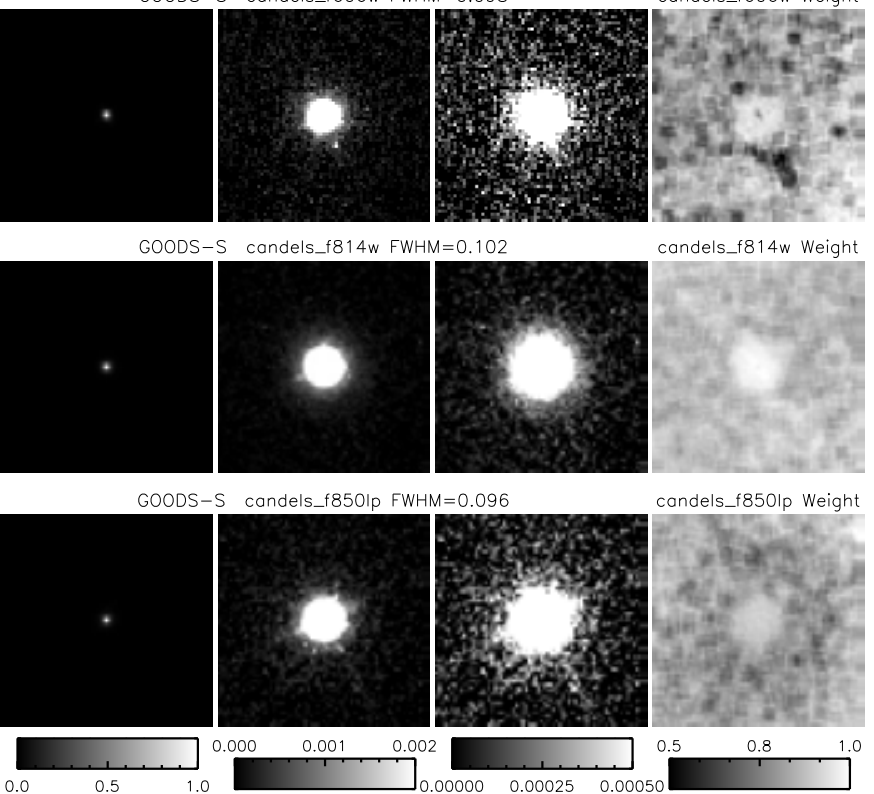
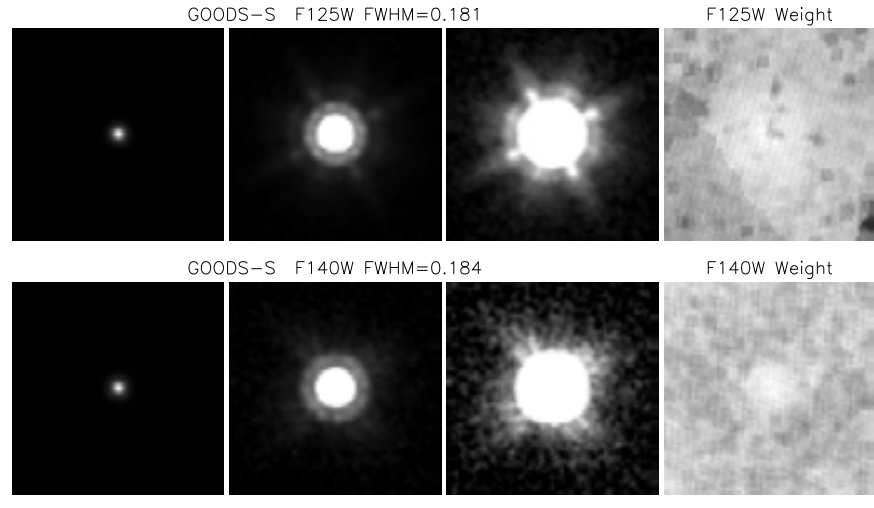

ODS $-S$ F140W FWHM $=0.184$
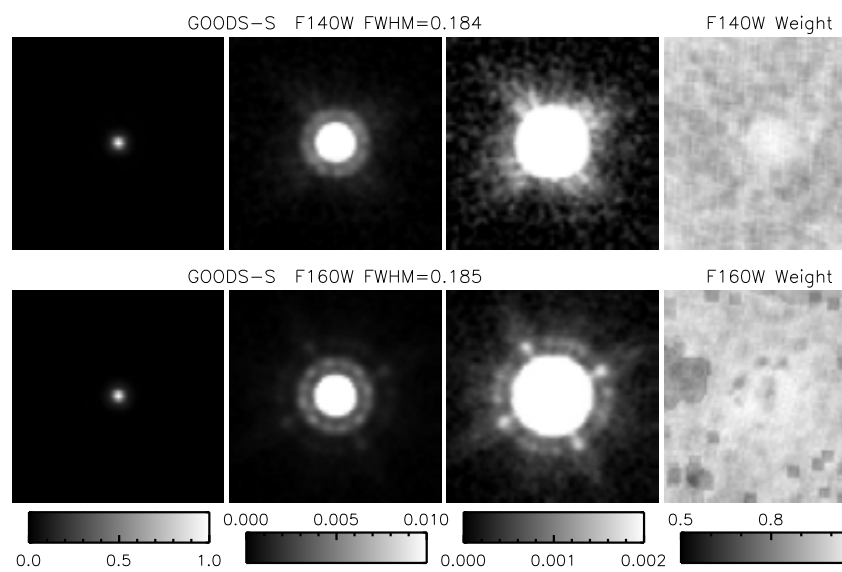

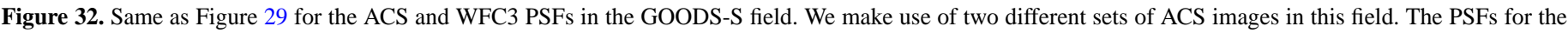
images from the GOODS Survey are shown in the top four rows (left). The PSFs for the images from the CANDELS Survey are shown in the bottom three rows.

of Ueda et al. (2008) are included in the UDS spectroscopic redshift catalog, and four of these are outliers in the spectroscopic versus photometric redshift comparison. Given the small numbers of redshifts in the UDS, excluding these X-ray sources has a noticeable impact, reducing the outlier fraction to $6 \%$. In GOODS-N there are approximately 45 sources in common with the Chandra $2 \mathrm{Ms}$ optically bright catalog from Alexander et al. (2003), however, very few of these are outliers. The relatively high fraction of outliers in this field is likely to be caused by unreliable spectroscopic redshifts, as there are no quality flags made available in the merged source catalog. This can be contrasted with the low outlier fraction in the AEGIS field, where the spectroscopic redshifts are uniformly sourced from the DEEP2+3 surveys and stringent quality cuts have been applied.

In Figure 24 we show the photometric redshift distributions of galaxies (selected with use_phot $=1$ ) in each of the 3D-HST fields, using z_mc from EAZY. $z_{-} m c$ is a "Monte Carlo" redshift, randomly chosen from the EAZY probability distribution for each galaxy and is more appropriate for showing redshift 

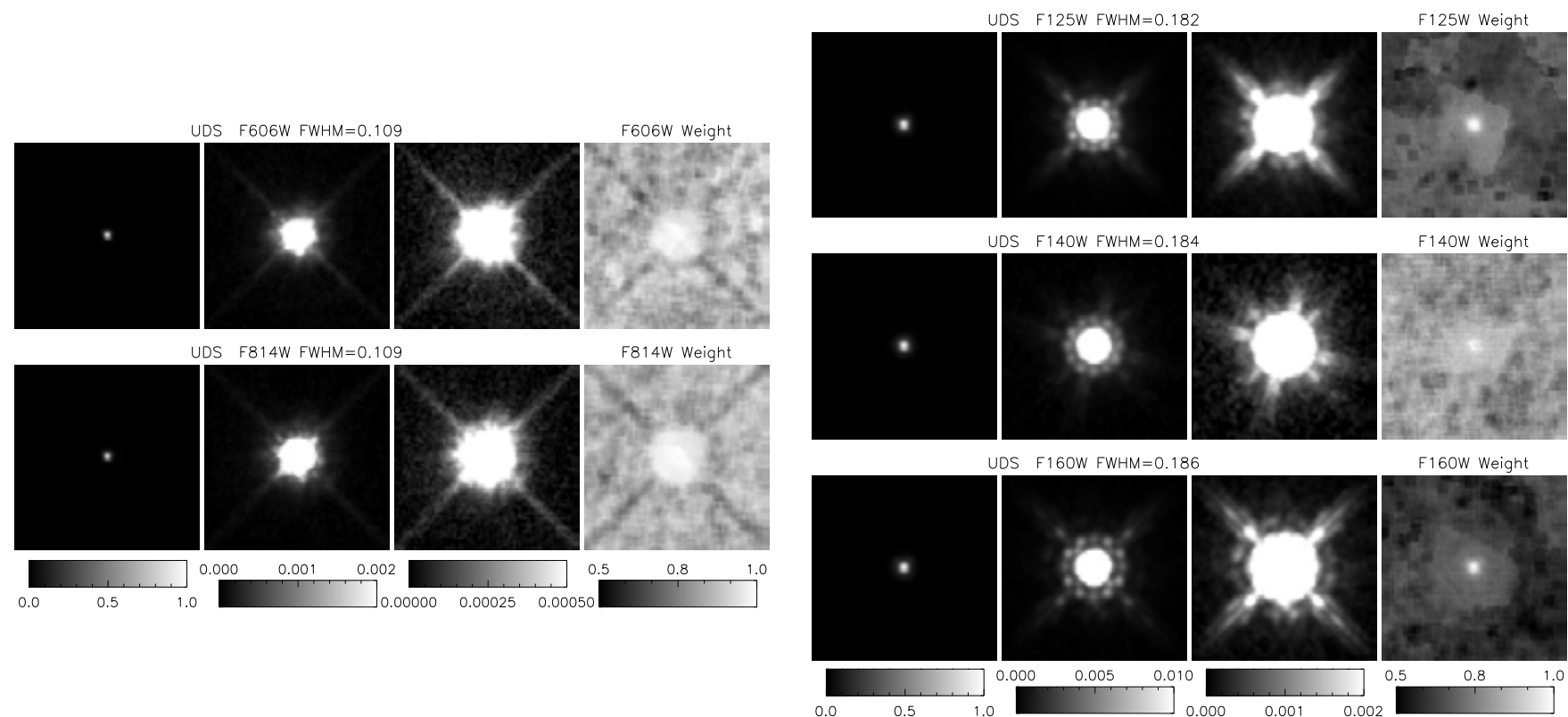

F140W Weight

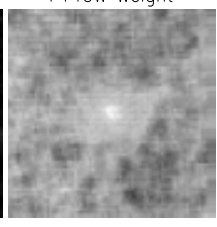

F160w Weight

Figure 33. Same as Figure 33 for the ACS and WFC3 PSFs in the UDS field.

distributions incorporating the redshift uncertainties (Wittman 2009). The spectroscopic redshift distributions are shown by the hashed histograms. Overdensities such as those already known at $z=0.7$ and $z=1.1$ in GOODS-S (Adami et al. 2005) and the $z=1.6$ cluster in the UDS (Papovich et al. 2010) stand out clearly. These overdensities can also be seen in the distribution of apparent magnitudes with $z_{-}$peak, shown in Figure 25, and in the mass distribution (see Figure 27 below). In the lower panel of Figure 25 we show the number of galaxies as a function of $z_{-}$peak, adding the contributions from each consecutive field to the total number in that redshift bin. The upper (black) histogram then shows the total number in all five fields, while the difference between the upper histogram and the one below it shows the number of galaxies in AEGIS, and so on for each field.

\subsection{Rest-frame Colors}

The catalogs contain colors of galaxies in the observed frame, but to compare galaxies at different redshifts rest-frame colors need to be used. These can be determined robustly as we have a wide range of observed-frame photometry in each of the fields. We use the EAZY templates and best-fitting redshift for each galaxy to determine its rest-frame luminosity in a series of filters, and determine rest-frame colors as the ratio of the luminosities in two filters. More information on how the rest-frame colors are calculated is provided in Brammer et al. (2011), but we note that the calculation is now made for individual filters, rather than a set of two filters. That is, the templates are refit forcing $z=z_{\text {phot }}$ and the flux in the rest-frame bandpass, $j$, is taken from the best-fit template considering only observed filters, $i$, where $\left|\lambda_{\text {obs }, \mathrm{i}}-\lambda_{\text {rest } \mathrm{j}}(1+z)\right|<1000 \AA$. We provide a catalog that contains the rest-frame luminosities in a variety of commonly used filters.

In order to assess the quality of the rest-frame photometry we show the "UVJ" diagram of galaxies in Figure 26. This diagram shows the rest-frame $U-V$ color versus the rest-frame $V-J$ color. Each column represents one of the fields, with redshift increasing from top to bottom, as shown in the top left corner of each panel. Galaxies (selected with use_phot = 1) are color-coded by mass, with the most massive galaxies in red $\left(\log M_{*}>11\right)$, galaxies with $10.5<\log M_{*}<11$ in orange, $10<\log M_{*}<10.5$ in green and $9<\log M_{*}<10$ in blue. The black lines mark the selection that is typically used to distinguish star-forming and quiescent galaxies (Williams et al. 2009; Whitaker et al. 2011). In this space, quiescent galaxies with low levels of star formation that are red in $U-V$ (upper left region) are separated from similarly red (in $U-V$ ), dusty star-forming galaxies, with the star-forming galaxies having redder $V-J$ colors. A clear progression of increasing mass toward redder colors can be seen along the quiescent galaxy sequence, particularly in the lowest redshift bins. The majority of low mass galaxies lie in the star-forming "blue cloud" at all redshifts. In the highest redshift bin $(2.5<z \leqslant 3.5)$ the most massive galaxies lie within the star-forming region and appear to be red due to higher levels of dust rather than older stellar populations.

In future papers we will interpret the distribution of galaxies in this color-color plane; here we simply note that the $U V J$ distributions are largely consistent from field to field. The only exception is the quiescent galaxy sequence in the UDS at $z \leqslant 1$, where the relation is tighter and the median somewhat bluer than the other fields. This is likely to be caused by the groundbased $u$-band data, where we found that a large zero point offset was required to make it consistent with the other bands (see Appendix B).

\subsection{Stellar Population Parameters}

We use the FAST code (Kriek et al. 2009) to estimate the stellar masses, star formation rates, ages and and dust extinctions, given the spectroscopic redshift, where available, or the photometric redshift from EAZY z_peak otherwise. We use the Bruzual \& Charlot (2003) stellar population synthesis model library with a Chabrier (2003) IMF and solar metallicity. We assume exponentially declining star formation histories with a minimum e-folding time of $\log _{10}(\tau / y r)=7$, a minimum age of 40 Myr, $0<A_{V}<4$ mag and the Calzetti et al. (2000) dust attenuation law. The stellar population parameters are provided in separate catalogs for each field. We stress that the star formation rates, dust absorption, and star formation 

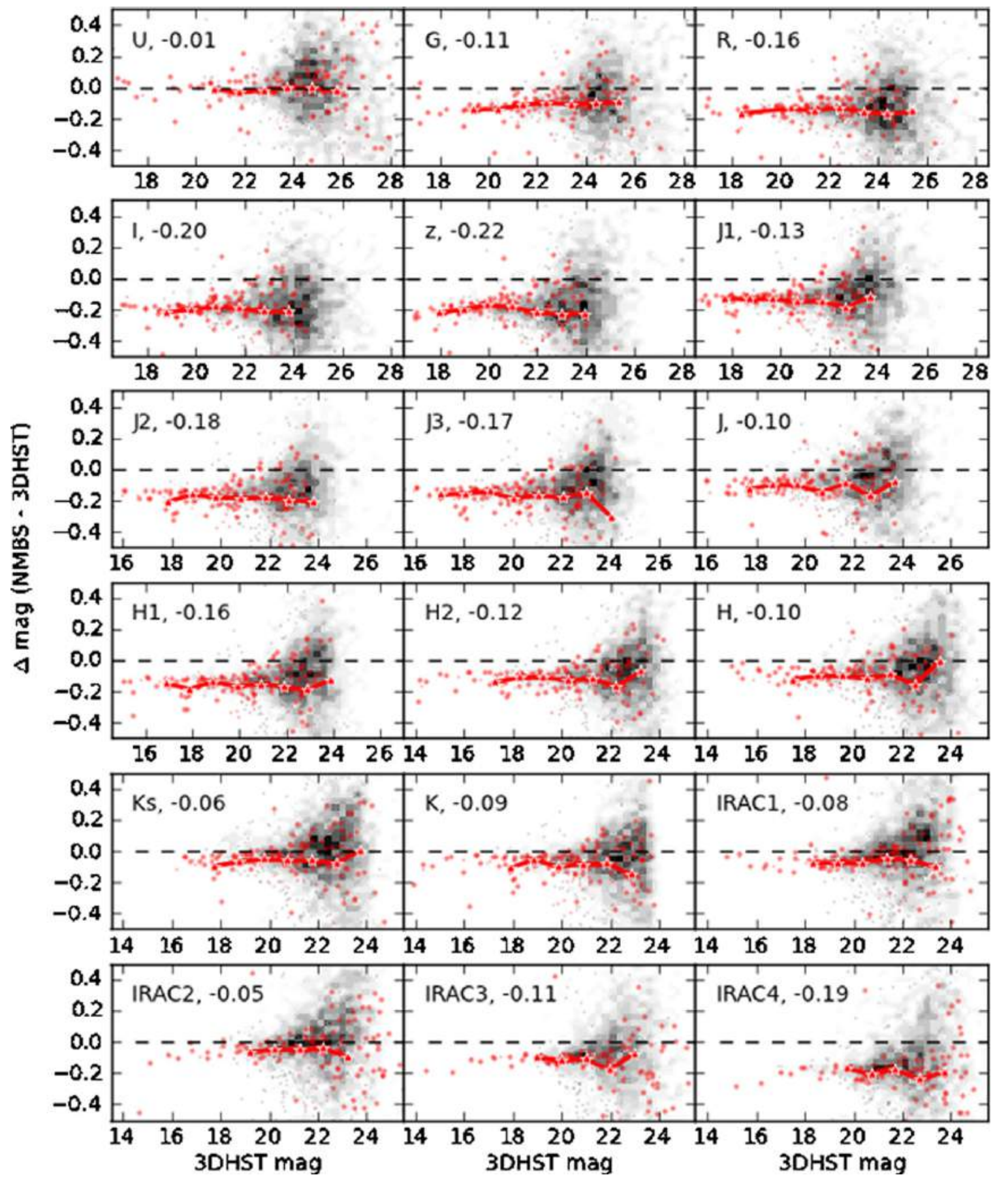

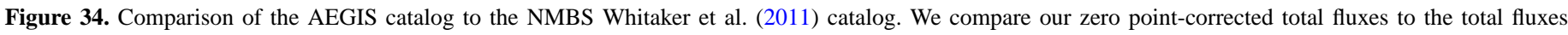

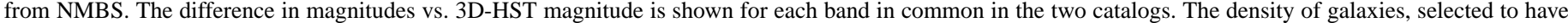

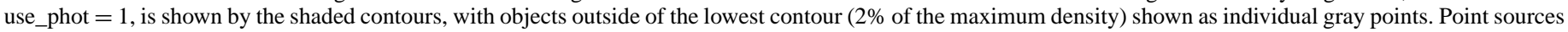

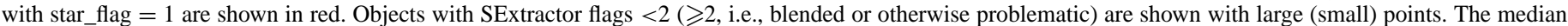
magnitude difference for all stars in bins of $1 \mathrm{mag}$ is shown by the red solid line and large red star symbols.

(A color version of this figure is available in the online journal.)

histories of the galaxies are uncertain when they are derived solely from optical - near-IR photometry (see, e.g., Wuyts et al. 2012). By contrast, stellar masses and $M / L$ ratios are relatively well-constrained as they mostly depend on the rest-frame optical colors of the galaxies, and these are well-covered by our photometry.

In Figure 27 we show the distribution of galaxy stellar masses with photometric redshift (z_peak). The points are color-coded according to the galaxy's $H_{\mathrm{F} 160 \mathrm{w}}$ magnitude, with the brightest galaxies in green $\left(H_{\mathrm{F} 160 \mathrm{~W}}<24\right)$, galaxies with $24 \leqslant H_{F 160 \mathrm{~W}}<$ 25 in blue and $25 \leqslant H_{F 160 W}<26$ in red. The histograms show the distribution of spectroscopic redshifts in each field (arbitrarily normalized). Many of the overdensities that can be seen in the photometric redshift distribution correspond to peaks in the distributions of already known spectroscopic redshifts, but extend to lower mass (fainter) galaxies than it 

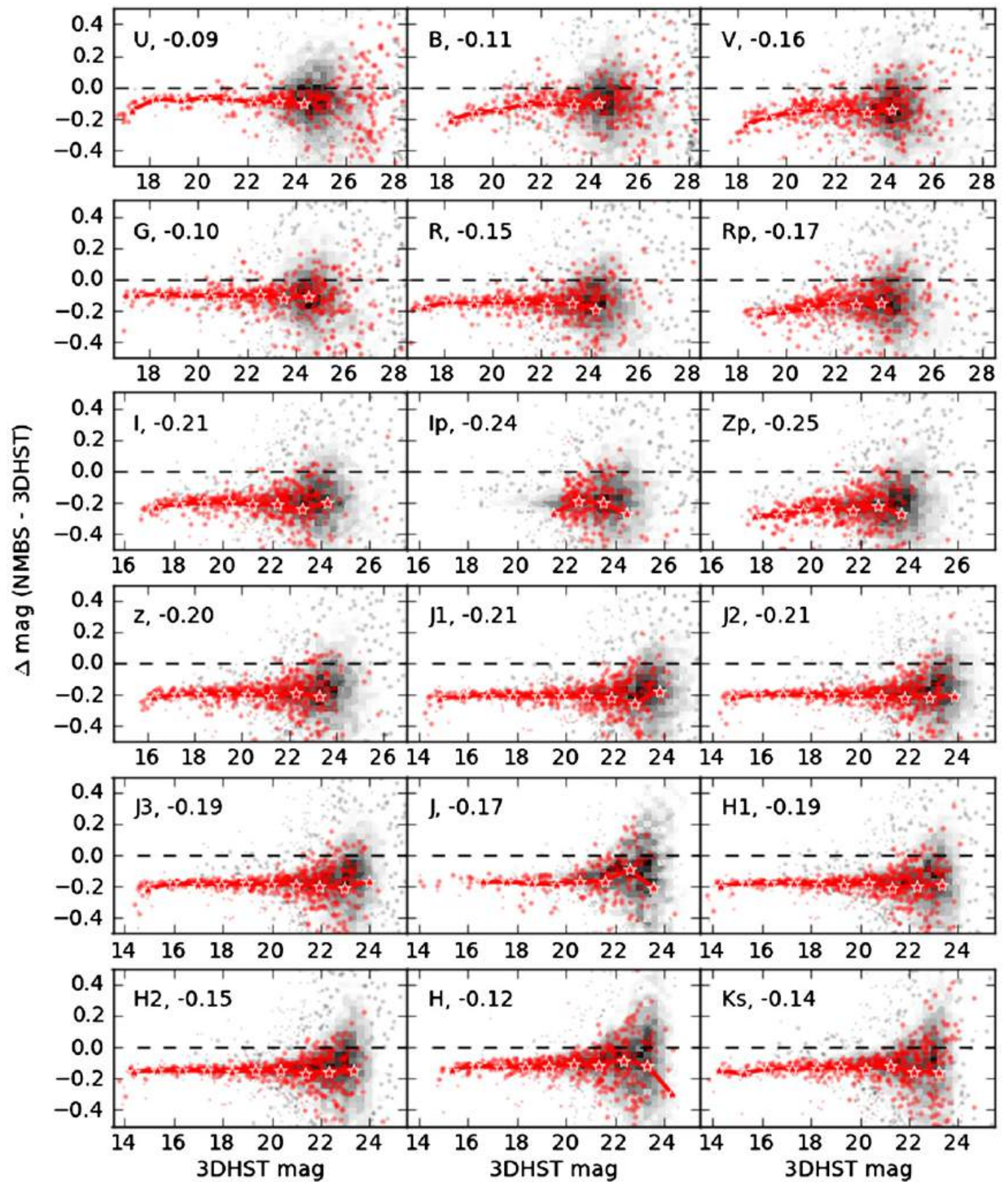

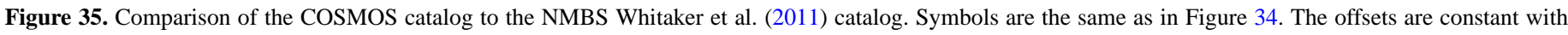
magnitude and can largely be explained by the zero point corrections we have made to adjust the ground-based data to the $H S T$ system.

(A color version of this figure is available in the online journal.)

is possible to measure spectroscopic redshifts for from the ground.

\section{SUMMARY}

In this paper we have presented the images and multiwavelength photometric catalogs produced by the 3D-HST project for the five CANDELS/3D-HST extragalactic fields. The survey covers $\sim 900 \mathrm{arcmin}^{2}$ in the AEGIS, COSMOS, GOODS-North, GOODS-South and UDS fields with HST/ WFC3 imaging and grism spectroscopy. The details of the WFC3 image reduction are given in Section 2.1.2. In addition to the new WFC3 data, we incorporated much of the available ground-based, Spitzer and HST/ACS data into the catalogs, using a total of 147 distinct data sets (see Section 2.2 and Table 3). We make all the images that have been used available on our website together with the catalogs. Each of the images is on the same astrometric system as the CANDELS WFC3 mosaics.

We have applied consistent methodology to produce multiwavelength catalogs for all five of the fields. The SExtractor software (Bertin \& Arnouts 1996) was used to detect sources on a noise-equalized combination of the $F 125 \mathrm{~W}, F 140 \mathrm{~W}$ and $F 160 \mathrm{~W}$ images. By using all three WFC 3 bands, we exploited the maximum survey area and depth. As described 

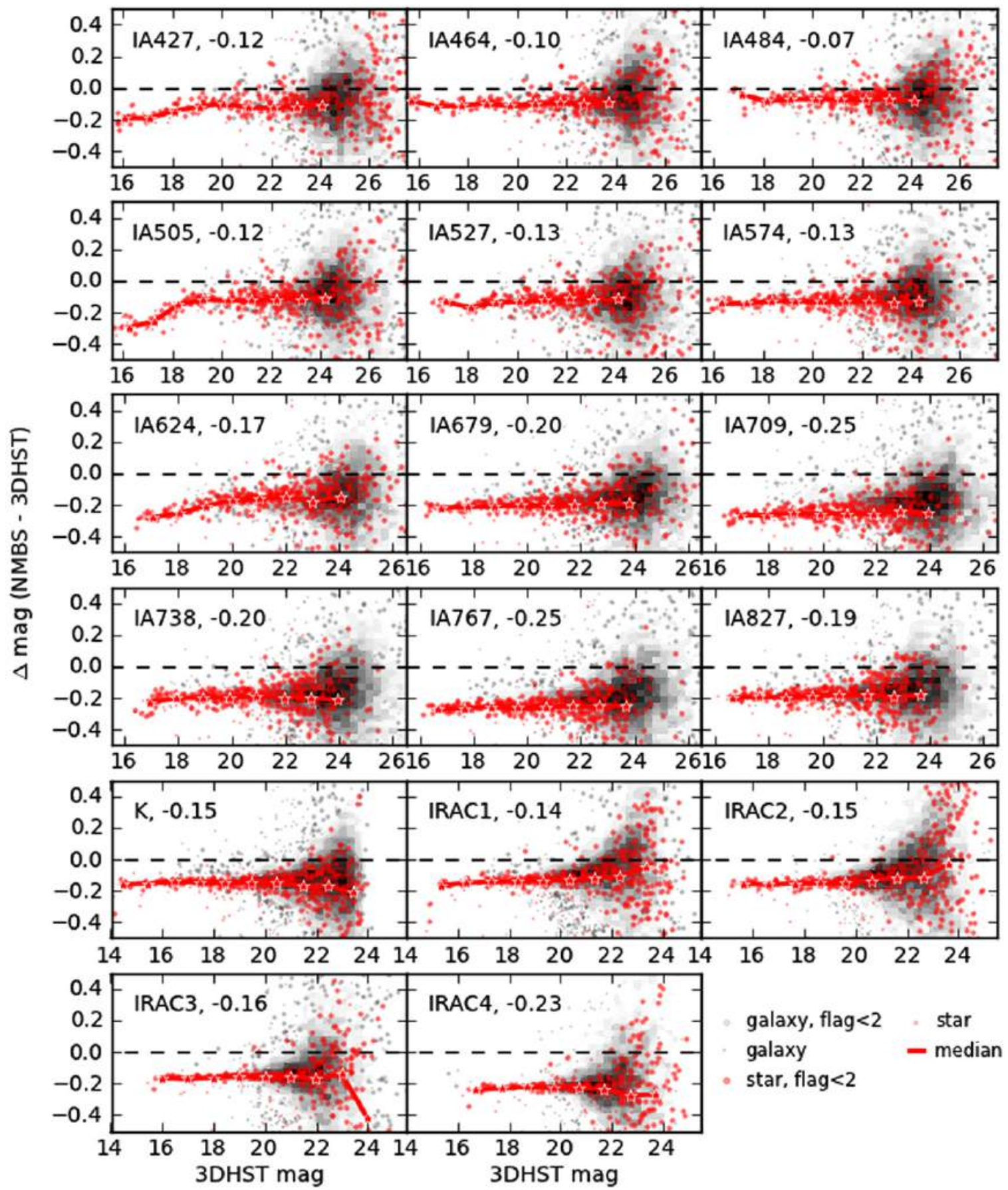

Figure 36. Comparison of the COSMOS catalog to the NMBS Whitaker et al. (2011) catalog continued. Symbols are the same as in Figure 34. (A color version of this figure is available in the online journal.)

in detail in Section 3, we measured the SEDs of objects using all the available ancillary data, carefully taking into account differences in image resolution (see Sections 3.4 and 3.5). The results are consistent for all five of the fields and the total WFC3 magnitudes agree well with independently derived total magnitudes from morphological fitting (see Section 4). The resulting SEDs span from the $U$-band to $8 \mu \mathrm{m}$ and are of excellent quality, as demonstrated throughout the paper. We used the EAZY code (Brammer et al. 2008) to fit photometric redshifts and reach an NMAD scatter between the photometric and spectroscopic redshifts of $<2.7 \%$ with fewer than $5 \%$ significant outliers in all fields. In the two fields where there is good medium band coverage (COSMOS and GOODS-S), the scatter is $\leqslant 1 \%$. We provide rest-frame colors based on the bestfitting EAZY templates, as well as stellar masses and stellar population parameters for all the galaxies based on fits to their SEDs.

The CANDELS team has provided similar catalogs for two of the five fields discussed in this paper (Guo et al. 2013; Galametz et al. 2013), and we can expect future CANDELS releases of the other three fields. Our catalogs are complementary to these; we use slightly deeper detection images and a larger number of photometric filters, but these differences are probably not critical for most purposes. It will be very useful to have multiple "realizations" of the CANDELS datasets in the public domain, using independent reductions and methodology. In an 

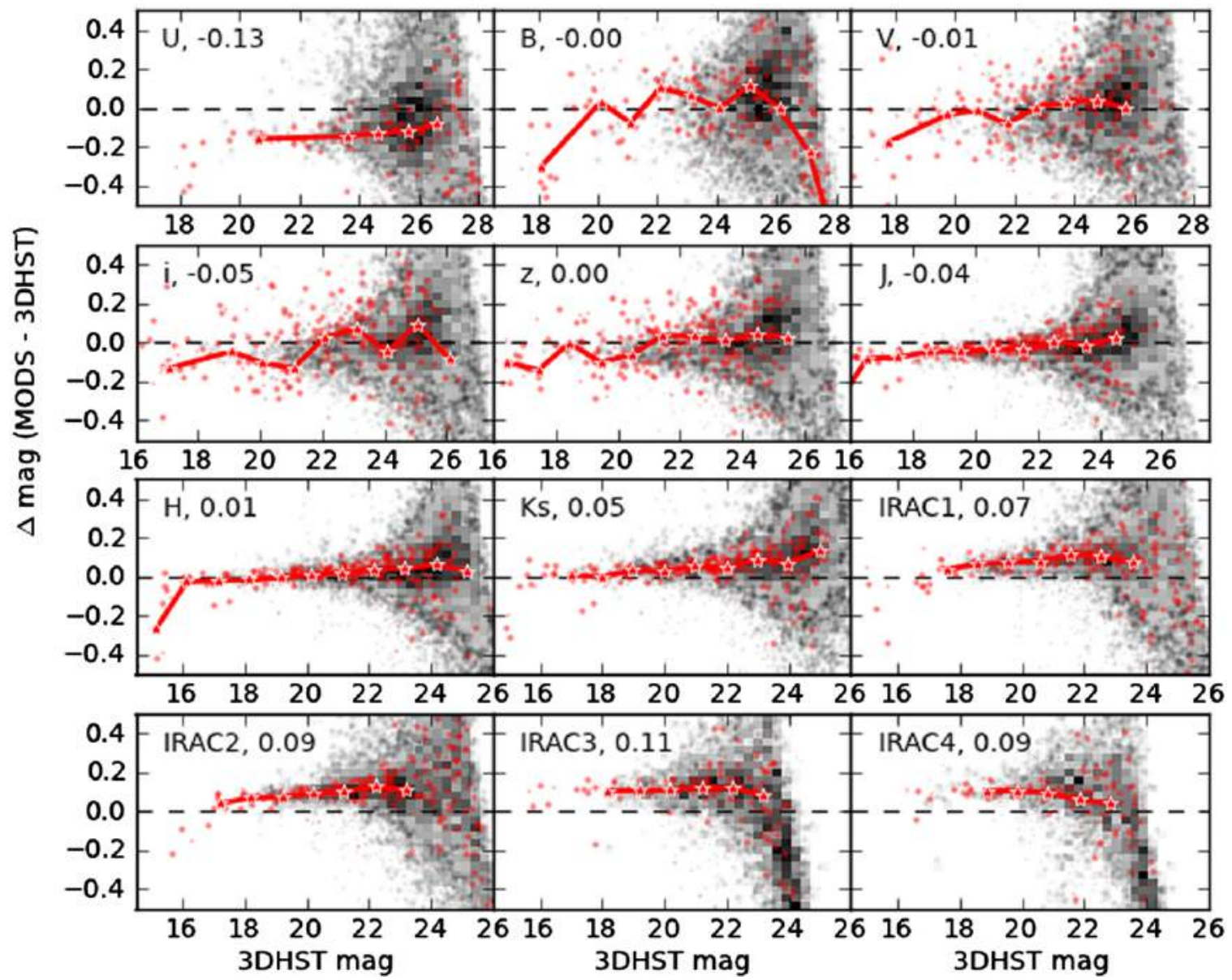

Figure 37. Comparison of the GOODS-N catalog to the Moircs Deep Survey catalog (Kajisawa et al. 2011). Symbols are the same as in Figure 34. (A color version of this figure is available in the online journal.)

Appendix we show band-by-band differences between the CANDELS catalogs and ours, and such comparisons provide much-needed estimates of systematic uncertainties in the various catalogs.

As explained in the Introduction, in the context of the 3DHST project, the work described in this paper merely concludes the first phase of an even more ambitious undertaking. The most innovative aspect of 3D-HST is the grism spectroscopy; future papers will describe these data and will quantify how they improve the measurement of redshifts, masses, and other parameters.

We are grateful to the many colleagues who have provided public data and catalogs in the five fields described in this paper; high redshift galaxy science has thrived owing to this gracious mindset and the TACs and Observatory Directors who have encouraged this. Formal acknowledgements follow below. R.S. acknowledges the support of the South African National Research Foundation through the Professional Development Programme Postdoctoral Fellowship. K.W. acknowledges support by an appointment to the NASA Postdoctoral Program at the Goddard Space Flight Center, administered by Oak Ridge Associated Universities through a contract with the National Aeronautics and Space Administration (NASA). D.M. acknowledges the support of the Research Corporation for Science Advancements Cottrell Scholarship. B.L. acknowledges support from the NSF Astronomy and Astrophsics Fellowship grant AST-1202963. We acknowledge support from
ERC Advanced Grant HIGHZ \#227749 and an NWO Spinoza Grant. We thank the Lorentz Center for its support and hospitality.

This work is based on observations made with the NASA/ ESA Hubble Space Telescope, obtained from the MAST Data Archive at the Space Telescope Science Institute, which is operated by the Association of Universities for Research in Astronomy, Inc., under NASA contract NAS 5-26555. Observations associated with the following GO and GTO programs were used: 12063, 12440, 12442, 12443, 12444, 12445, 12060, 12061, 12062, 12064 (PI: Faber); 12177 and 12328 (PI: van Dokkum); 12461 and 12099 (PI: Riess); 11600 (PI: Weiner); 9425 and 9583 (PI: Giavalisco); 12190 (PI: Koekemoer); 11359 and 11360 (PI: OConnell); 11563 (PI: Illingworth). Based, in part, on data obtained at the W. M. Keck Observatory, which is operated as a scientific partnership among the California Institute of Technology, the University of California, and NASA and was made possible by the generous financial support of the W. M. Keck Foundation. This work makes use of data obtained as part of the ESO/GOODS survey. Observations have been carried out using the Very Large Telescope at the ESO Paranal Observatory under Programme ID 168.A-0485. Based on observations made with ESO Telescopes at the La Silla or Paranal Observatories under programme number 168.A-0485. Based on data products from observations made with ESO Telescopes at the La Silla Paranal Observatory under ESO programme ID 179.A-2005 and on data products produced by TERAPIX and the Cambridge Astronomy Survey Unit on behalf of the UltraVISTA 

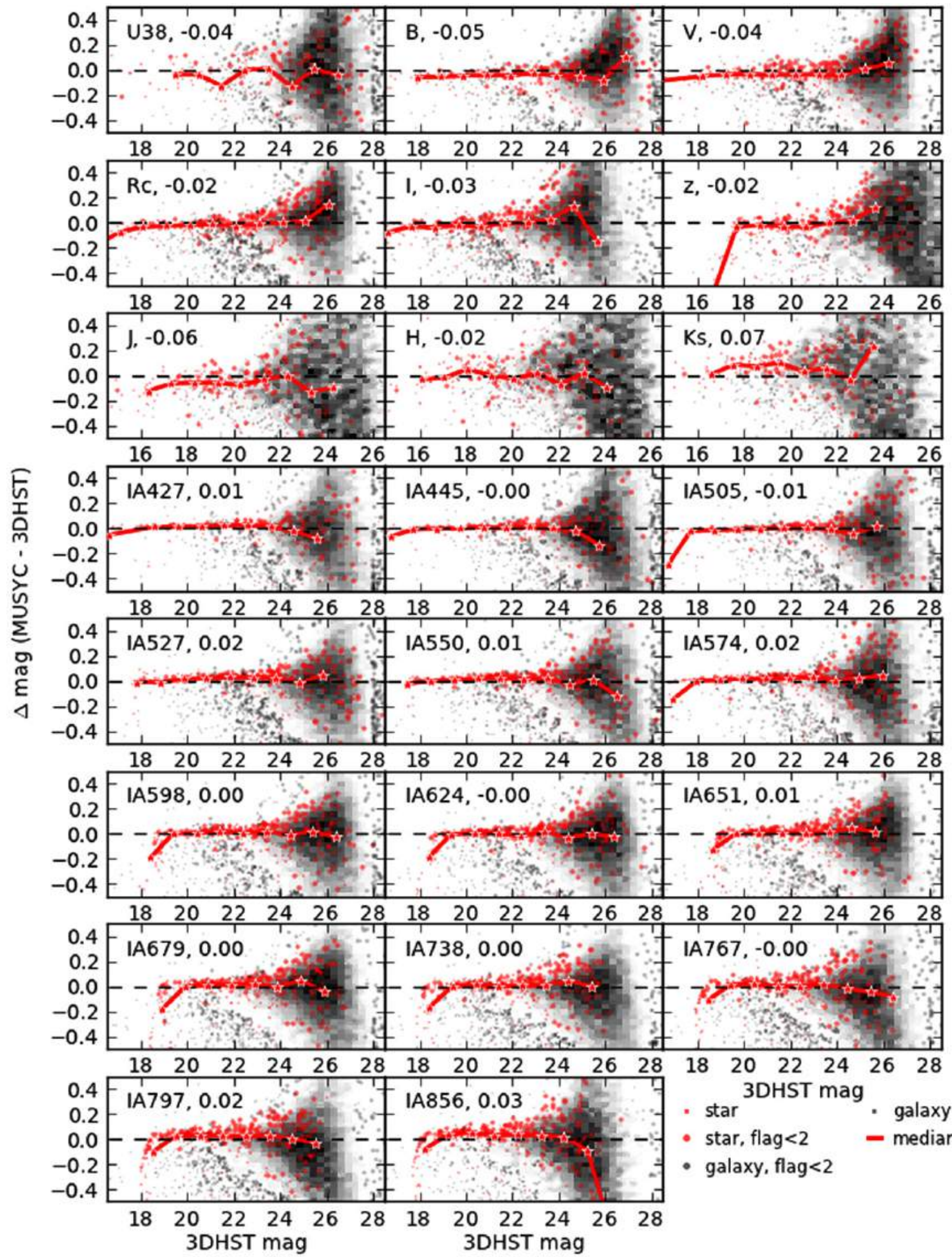

- star galaxy

- star, flag<2 - median

- galaxy, flag<2

Figure 38. Comparison of the GOODS-S catalog to the MUSYC catalog (Cardamone et al. 2010). We have applied the extinction and color corrections provided with the MUSYC release to their fluxes and compare to our total zero point-corrected fluxes. The agreement for stars is good in all the medium bands.

(A color version of this figure is available in the online journal.)

consortium. Based on observations obtained with MegaPrime/ MegaCam, a joint project of CFHT and CEA/IRFU, at the Canada-France-Hawaii Telescope (CFHT) which is operated by the National Research Council (NRC) of Canada, the Institut National des Science de l'Univers of the Centre National de la Recherche Scientifique (CNRS) of France, and the University of Hawaii. This work is based in part on data products produced at Terapix available at the Canadian Astronomy Data Centre as part of the Canada-France-Hawaii Telescope Legacy Survey, a collaborative project of NRC and CNRS. This work makes use of data products produced at TERAPIX, the WIRDS consortium, and the Canadian Astronomy Data Centre. We thank H. Hildebrandt for providing the CARS-reduced CFHTLS images. This research has made use of the NASA/IPAC Infrared 

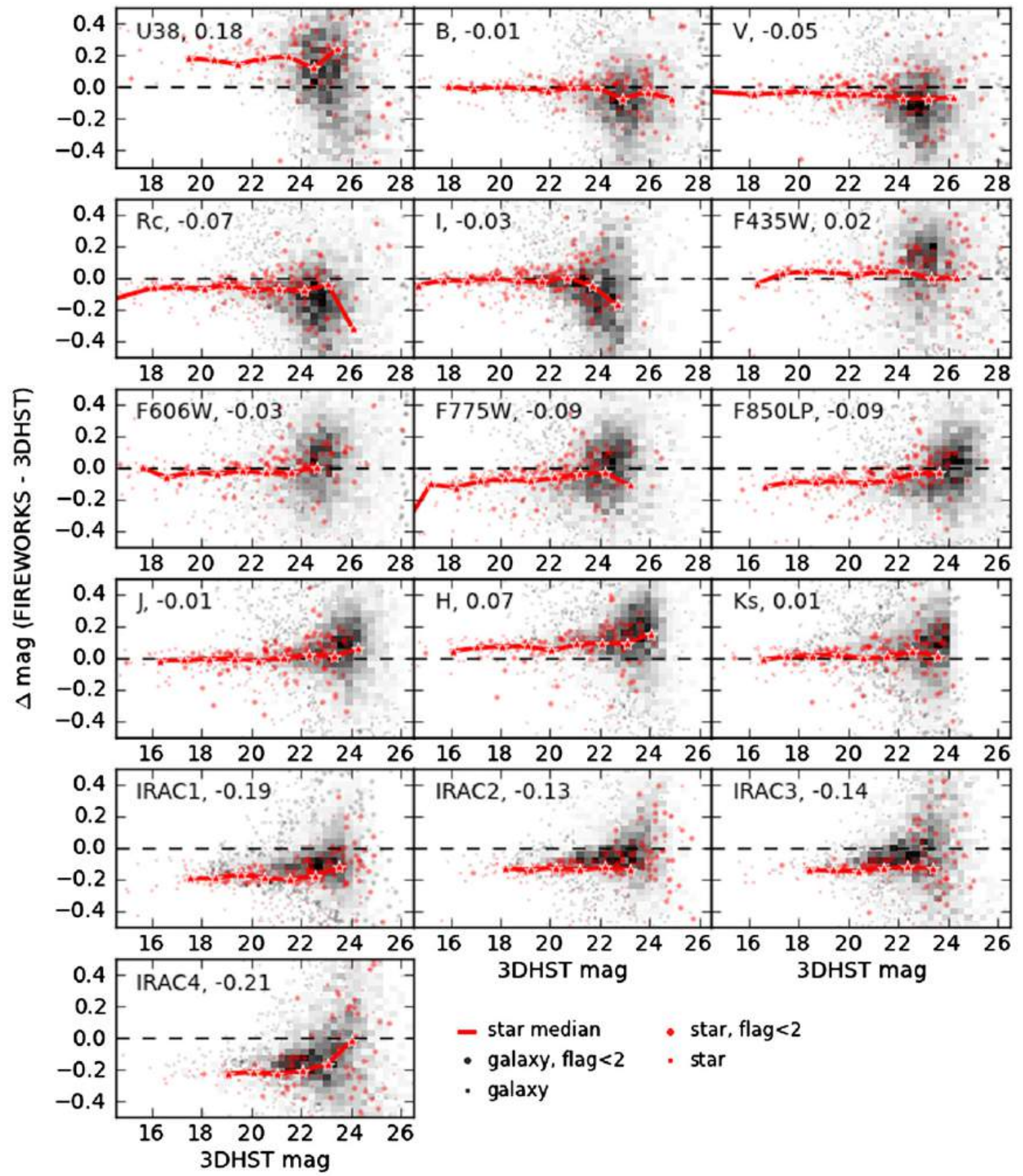

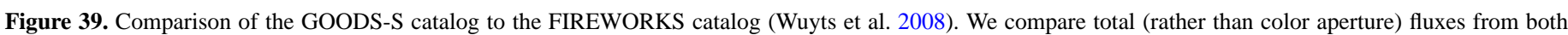

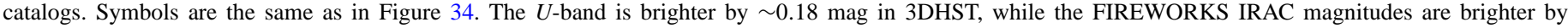
$\sim 0.1-0.2$ mag. There are no significant trends with magnitude.

(A color version of this figure is available in the online journal.)

Science Archive, which is operated by the Jet Propulsion Laboratory, California Institute of Technology, under contract with the National Aeronautics and Space Administration. This study makes use of data from AEGIS, a multiwavelength sky survey conducted with the Chandra, GALEX, Hubble, Keck, CFHT, MMT, Subaru, Palomar, Spitzer, VLA, and other telescopes and supported in part by the NSF, NASA, and the STFC. This study makes use of data from the NEWFIRM MediumBand Survey, a multi-wavelength survey conducted with the NEWFIRM instrument at the KPNO, supported in part by the NSF and NASA. This paper uses data products produced by the OIR Telescope Data Center, supported by the Smithsonian Astrophysical Observatory. This work is based on observations made with the Spitzer Space Telescope, which is operated by the Jet Propulsion Laboratory, California Institute of Technology under contract with NASA. Based in part on data from the UKIDSS Ultra Deep Survey (UDS) and the Spitzer Public Legacy Survey of the UKIDSS Ultra Deep Survey (SpUDS), a Cycle 4 Spitzer Legacy program. Author contributions: R.S. and K.W. led this part of the 3D-HST project and created the photometric catalogs described in this paper. R.S., I.M. and P.v.D. wrote the manuscript. I.M. is the 3D-HST project lead, reduced 

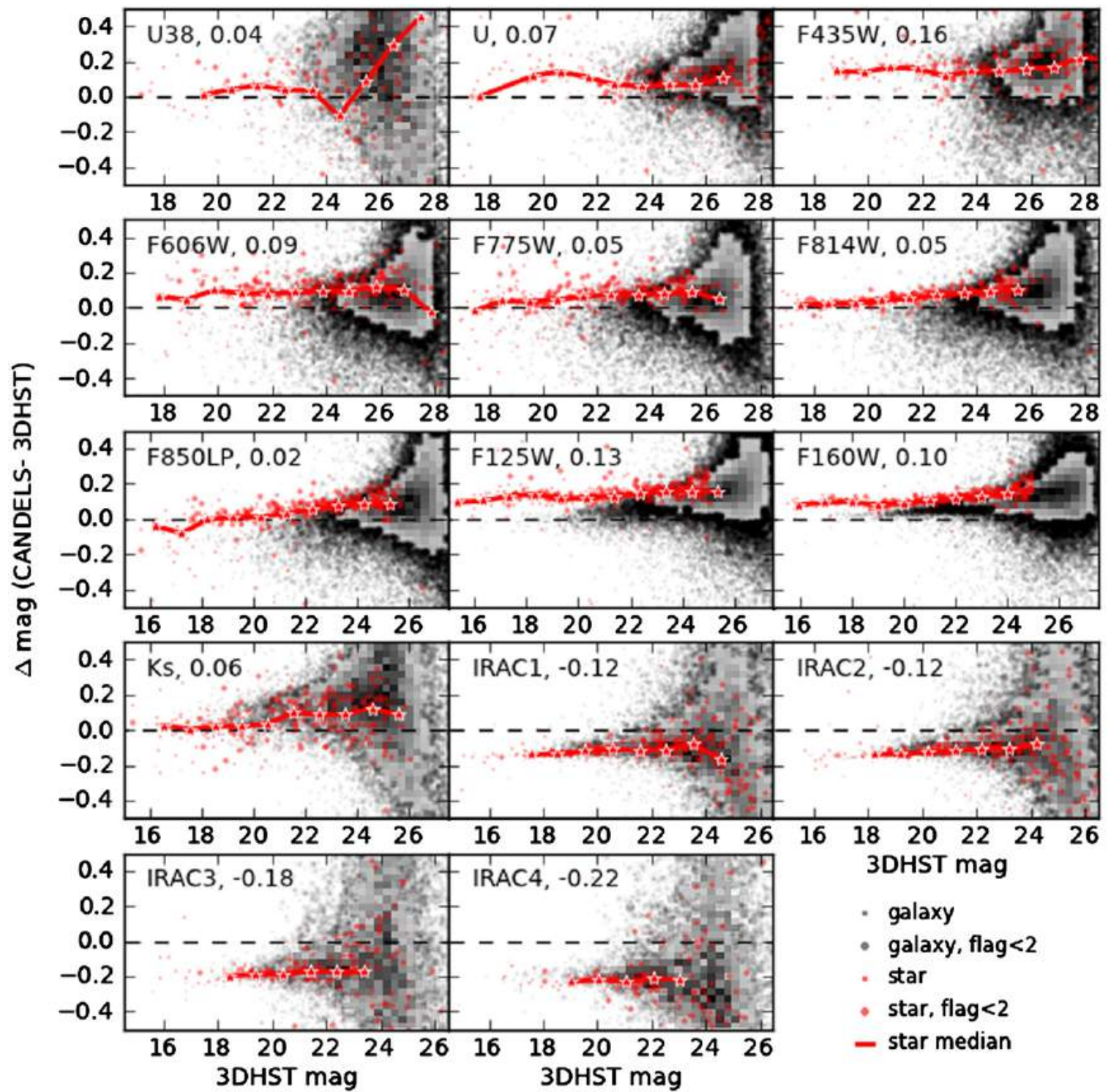

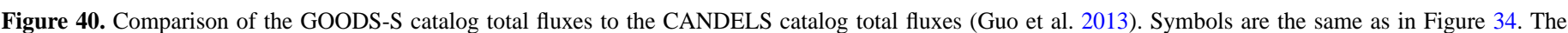

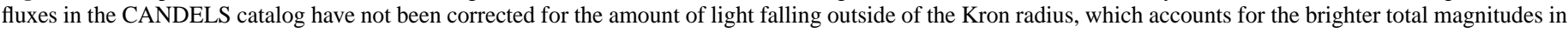
3D-HST.

(A color version of this figure is available in the online journal.)

all the WFC3 imaging in the CANDELS fields, and was responsible for the integration of the data products. G.B. and I.L. developed many of the algorithms and techniques used in the creation of the catalogs. P.v.D. is the PI of 3D-HST. The other authors provided help at various stages with the project and/or commented on the manuscript.

\section{APPENDIX A}

\section{POINT-SPREAD FUNCTIONS}

In the left-hand panel of Figure 28 we compare the growth curves of the $F 125 W$ and $F 160 W$ PSFs obtained using the same method (described in Section 3.3) from the 3D-HST (solid lines) and CANDELS v1.0 mosaics (dash-dot lines) to the hybrid PSF used for morphological fitting in van der Wel et al. (2012) (dotted lines) in the COSMOS field. The three PSFs in each band are very similar over the full range of apertures, with differences of $\lesssim 1 \%$ at apertures $>0$.' 5 . In the inner regions the hybrid PSF has larger flux than the 3D-HST PSF, indicating that we may still be missing the some flux due to the cosmic ray rejection. The CANDELS PSF has lower flux than the 3D-HST PSF in the cores. In the right-hand panel we show the growth curves for PSFs created using stars in bins of 1 mag in the COSMOS field. There is excellent agreement across the full range in magnitude, with only the very brightest stars $\left(H_{F 160 W}<14\right)$ having significantly less flux within the central region. Tests on the other fields yield similar results.

In Figures 29-33 we show the thumbnails of the PSFs and corresponding weight images for all the HST bands in all five fields. Each image is $69 \times 69$ pixels or 4 ". $14 \times 4$ ". 14 , i.e., it traces the PSF out to just over $2^{\prime \prime}$ radius. For each filter we show three stretch levels (panels 1-3 and 5-7) to expose the structure of the PSF: the core, the first Airy ring and the diffraction spikes. The images are normalized to a maximum value of one. The grayscale bars show the stretch for each panel. These are slightly different for ACS and WFC3 as a result of the different 
Table 11

Zero Point Offsets Applied to the v4.1 Catalogs

\begin{tabular}{|c|c|c|c|c|c|c|c|}
\hline Field & Band & Flux Correction & Magnitude Offset & Field & Band & Flux Correction & Magnitude Offset \\
\hline \multirow[t]{23}{*}{$\overline{\text { AEGIS }}$} & $U$ & 1.2258 & -0.22 & \multirow[t]{45}{*}{ COSMOS } & $U$ & 1.1624 & -0.16 \\
\hline & $G$ & 1.0076 & -0.01 & & $B$ & 0.9747 & 0.03 \\
\hline & $F 606 W$ & 0.9999 & 0.00 & & $G$ & 0.9436 & 0.06 \\
\hline & $R$ & 0.9594 & 0.05 & & V & 0.8055 & 0.23 \\
\hline & $I$ & 0.9065 & 0.11 & & $F 606 \mathrm{~W}$ & 1.0109 & -0.01 \\
\hline & $F 814 W$ & 0.9353 & 0.07 & & $R p$ & 0.8305 & 0.20 \\
\hline & Z & 0.8980 & 0.12 & & $R$ & 0.9239 & 0.09 \\
\hline & $J 1$ & 0.8568 & 0.17 & & $I p$ & 1.2181 & 0.12 \\
\hline & $J 2$ & 0.8749 & 0.15 & & $I$ & 0.8799 & 0.14 \\
\hline & $J 3$ & 0.8863 & 0.13 & & $F 814 W$ & 0.9658 & 0.04 \\
\hline & $J$ & 1.0575 & -0.06 & & Z & 0.8644 & 0.16 \\
\hline & $F 125 W$ & 0.9966 & 0.00 & & $Z p$ & 0.8232 & 0.21 \\
\hline & $H 1$ & 0.8899 & 0.13 & & UVISTA Y & 0.9152 & 0.10 \\
\hline & $H 2$ & 0.9196 & 0.09 & & $J 1$ & 0.8461 & 0.18 \\
\hline & $H$ & 1.0028 & 0.00 & & $J 2$ & 0.8593 & 0.16 \\
\hline & $F 140 W$ & 0.9967 & 0.00 & & $J 3$ & 0.8834 & 0.13 \\
\hline & $F 160 W$ & 1.0000 & 0.00 & & $J$ & 0.9290 & 0.08 \\
\hline & K & 0.9572 & 0.05 & & UVISTA J & 0.9284 & 0.08 \\
\hline & $K s$ & 0.9817 & 0.02 & & $F 125 W$ & 1.0129 & -0.01 \\
\hline & IRAC1 & 1.0142 & -0.02 & & $H 1$ & 0.8766 & 0.14 \\
\hline & IRAC2 & 0.9918 & 0.01 & & $H 2$ & 0.8866 & 0.13 \\
\hline & IRAC3 & 1.0453 & -0.05 & & $H$ & 0.9372 & 0.07 \\
\hline & IRAC4 & 1.0234 & -0.03 & & UVISTA H & 0.9860 & 0.02 \\
\hline \multirow[t]{22}{*}{ GOODS-N } & $U$ & 0.8324 & 0.20 & & $F 140 W$ & 1.0344 & -0.04 \\
\hline & B & 0.7878 & 0.26 & & $F 160 W$ & 1.0000 & 0.00 \\
\hline & $F 435 W$ & 1.0237 & -0.03 & & K & 0.8918 & 0.12 \\
\hline & $G$ & 0.9716 & 0.03 & & $K s$ & 0.9398 & 0.07 \\
\hline & V & 0.7966 & 0.25 & & UVISTA Ks & 0.9580 & 0.05 \\
\hline & $F 606 \mathrm{~W}$ & 1.0000 & 0.00 & & IRAC1 & 1.0170 & -0.02 \\
\hline & $R s$ & 0.9497 & 0.06 & & IRAC2 & 0.9724 & 0.03 \\
\hline & $R$ & 0.7244 & 0.35 & & IRAC3 & 0.9581 & 0.05 \\
\hline & I & 0.7702 & 0.28 & & IRAC4 & 0.8917 & 0.12 \\
\hline & $F 775 \mathrm{~W}$ & 0.9899 & 0.01 & & IA427 & 1.0314 & -0.03 \\
\hline & Z & 0.8524 & 0.17 & & IA464 & 1.0382 & -0.04 \\
\hline & $F 850 L P$ & 0.9877 & 0.01 & & IA484 & 0.9929 & 0.01 \\
\hline & $F 125 W$ & 1.0099 & -0.01 & & IA505 & 0.9497 & 0.06 \\
\hline & $J$ & 0.9050 & 0.11 & & IA527 & 0.9419 & 0.06 \\
\hline & $H$ & 0.9924 & 0.01 & & IA574 & 0.9695 & 0.03 \\
\hline & $F 140 \mathrm{~W}$ & 1.0129 & -0.01 & & IA624 & 0.7890 & 0.26 \\
\hline & $F 160 \mathrm{~W}$ & 1.0000 & 0.00 & & IA679 & 0.6997 & 0.39 \\
\hline & Ks & 1.0009 & 0.00 & & IA709 & 0.9086 & 0.10 \\
\hline & IRAC1 & 0.9889 & 0.01 & & IA738 & 0.9501 & 0.06 \\
\hline & IRAC2 & 0.9984 & 0.00 & & IA767 & 0.8625 & 0.16 \\
\hline & IRAC3 & 1.0891 & -0.09 & & IA 827 & 0.9712 & 0.03 \\
\hline & IRAC4 & 1.0799 & -0.08 & & & & \\
\hline \multirow[t]{20}{*}{ GOODS-S } & $U$ & 1.0846 & -0.09 & \multirow[t]{20}{*}{ UDS } & $u$ & 1.2635 & -0.25 \\
\hline & U38 & 1.2200 & -0.22 & & $B$ & 0.9756 & 0.03 \\
\hline & B & 1.0055 & -0.01 & & V & 0.9472 & 0.06 \\
\hline & $F 435 \mathrm{~W}$ & 1.0819 & -0.09 & & $F 606 \mathrm{~W}$ & 1.0079 & -0.01 \\
\hline & V & 0.9787 & 0.02 & & $R$ & 0.8442 & 0.18 \\
\hline & $F 606 W$ cand & 1.0038 & 0.00 & & $i$ & 0.7961 & 0.25 \\
\hline & $F 606 W$ & 1.0033 & 0.00 & & $F 814 W$ & 0.9342 & 0.07 \\
\hline & $R$ & 1.0185 & -0.02 & & $z$ & 0.8448 & 0.18 \\
\hline & $R c$ & 0.9367 & 0.07 & & $F 125 \mathrm{~W}$ & 1.0048 & -0.01 \\
\hline & $F 775 \mathrm{~W}$ & 0.9845 & 0.02 & & $J$ & 1.0096 & -0.01 \\
\hline & I & 0.9910 & 0.01 & & $H$ & 1.0549 & -0.06 \\
\hline & $F 814 W$ cand & 0.9919 & 0.01 & & $F 140 \mathrm{~W}$ & 1.0257 & -0.03 \\
\hline & $F 850 L P$ & 0.9838 & 0.02 & & $F 160 W$ & 1.0000 & 0.00 \\
\hline & $F 850 L P$ cand & 1.0022 & 0.00 & & K & 1.0614 & -0.06 \\
\hline & $F 125 W$ & 1.0028 & -0.00 & & IRAC1 & 1.0426 & -0.05 \\
\hline & $J$ & 0.9975 & 0.00 & & IRAC2 & 1.0041 & 0.00 \\
\hline & tenisJ & 0.8736 & 0.15 & & IRAC3 & 1.1500 & -0.15 \\
\hline & $H$ & 1.0752 & -0.08 & & IRAC4 & 1.1500 & -0.15 \\
\hline & $F 140 \mathrm{~W}$ & 1.0072 & -0.01 & & & & \\
\hline & $F 160 W$ & 1.0000 & 0.00 & & & & \\
\hline
\end{tabular}


Table 11

(Continued)

\begin{tabular}{|c|c|c|c|c|c|c|c|}
\hline Field & Band & Flux Correction & Magnitude Offset & Field & Band & Flux Correction & Magnitude Offset \\
\hline & tenisK & 0.7644 & 0.29 & & & & \\
\hline & $K s$ & 1.0360 & -0.04 & & & & \\
\hline & IRAC1 & 1.0226 & -0.02 & & & & \\
\hline & IRAC2 & 1.0124 & -0.01 & & & & \\
\hline & IRAC3 & 0.9693 & 0.03 & & & & \\
\hline & IRAC4 & 0.9451 & 0.06 & & & & \\
\hline & IA427 & 0.9815 & 0.02 & & & & \\
\hline & IA445 & 0.9839 & 0.02 & & & & \\
\hline & IA505 & 0.9861 & 0.02 & & & & \\
\hline & IA527 & 0.9519 & 0.05 & & & & \\
\hline & IA550 & 0.9632 & 0.04 & & & & \\
\hline & IA574 & 1.0385 & -0.04 & & & & \\
\hline & IA598 & 0.9468 & 0.06 & & & & \\
\hline & IA624 & 0.8999 & 0.11 & & & & \\
\hline & IA651 & 0.9798 & 0.02 & & & & \\
\hline & IA679 & 0.9991 & 0.00 & & & & \\
\hline & IA738 & 0.9228 & 0.09 & & & & \\
\hline & IA767 & 0.9092 & 0.10 & & & & \\
\hline & IA797 & 0.9102 & 0.10 & & & & \\
\hline & IA856 & 0.8628 & 0.16 & & & & \\
\hline
\end{tabular}

Notes. Corrected_AB $=25-2.5 \log _{10}($ Flux $\times$ Flux correction $)$ or Corrected_AB $=25-2.5 \log _{10}$ (Flux)+magnitude offset.

FWHMs (listed above the images). We also show the combined weight images for each PSF. The weight is largest in the center and lower at larger radii due to masking of neighboring objects. The ACS PSFs have lower weights in the central pixels because of cosmic ray rejection flagging the centers of stars.

\section{APPENDIX B}

\section{ZERO POINT OFFSETS FROM SED FITTING}

When fitting photometric redshifts, we apply two corrections: we modify the templates and we modify the photometric zero points. These two corrections are separable, as the template correction is derived in the rest-frame and the zero point correction is derived in the observed frame. As we have many objects and many filters we can robustly determine the required corrections. In detail, we shift the observed and best-fit SEDs to the rest-frame and examine differences between the two for a large number of objects as a function of wavelength. We then improve the templates to include subtle features that are not included in the models, such as the broad dust absorption feature at $2175 \AA$. Since the galaxies span a wide range of redshifts, shifting to the rest-frame ensures that each part of the spectrum is sampled by a number of different photometric bands. This allows one to disentangle template effects from systematic offsets between the photometric bands. After adjusting the templates we fit for a photometric zero point offset for each filter from the residuals in an iterative fashion. We largely follow the procedure described in Whitaker et al. (2011) and other works, but with two significant differences. We now include all objects in the fit rather than just galaxies that have spectroscopic redshifts. By including all objects, we avoid the bias toward lower redshift star-forming galaxies which dominate the sample with spectroscopic redshifts and are able to obtain good estimates of the zero point offsets even in fields with limited numbers of spectroscopic redshifts. Additionally we follow a two-step process to account for the relatively large zero point uncertainties in the ground-based data compared to the well-calibrated HST filters. In the first iteration we allow the $H S T$ bands to vary with respect to each other, keeping $H_{F 160 W}$ as a fixed reference point. The HST band zero points are then fixed and the ground-based and IRAC band zero points are allowed to vary until convergence is reached. Convergence is defined as having the largest change in any band other than $U$ or IRAC be less than $0.5 \%$, and this condition is usually met after only three or four iterations of the procedure.

We list the offsets applied to the fluxes in each field in Table 11. The zero point offsets for the HST bands are generally small, of the order of $0.01 \mathrm{mag}$. It is hardest to separate template and photometric zero point errors where there are no bracketing filters at the bluest and reddest ends of the spectra, sampled by the $U$-band and IRAC $8 \mu \mathrm{m}$, respectively. The COSMOS CFHTLS $u$-band zero point is known to be highly uncertain (Erben et al. 2009; Whitaker et al. 2011) as is the UDS $u^{\prime}$ band (R. Quadri 2012, private communication). As a result, the zero point offsets applied in the $U$-band can be as large as $0.25 \mathrm{mag}$. The IRAC offsets are $\leqslant 0.15 \mathrm{mag}$ in the $8 \mu \mathrm{m}$ band and generally much smaller in the other channels. The GOODS$\mathrm{N}$ optical Subaru data are offset from the other optical data by $\sim 0.2$ mag. Some of the optical medium bands with large FWHM in GOODS-S were found to have particularly large offsets and were excluded from further analysis (IA464, IA484, IA709, IA827). These bands are not included in the released catalogs.

In the COSMOS field there appears to be a systematic difference between the ground-based and the space-based NIR data, with an average offset of $0.1 \mathrm{mag}$. Similar offsets are found in the AEGIS $J$ bands. The $K$ bands from the NMBS, WIRDS and UltraVISTA are known to have differences in the calibrated zero point (see the discussion in the Appendix of Muzzin et al. 2013). In both NMBS and UltraVISTA, the difference between the measured fluxes for NMBS $K$ and WIRDS $K_{s}$ was found to be $\sim 0.03 \mathrm{mag}$. In the NMBS COSMOS catalog a zero point offset of $0.05 \mathrm{mag}$ is applied to the WIRDS $K_{s}$-band to bring it into agreement with the NMBS $K$ band. Muzzin et al. (2013) find a difference a difference of 0.08 mag between the NMBS $K$ and UltraVISTA $K_{s}$, such that the NMBS fluxes are brighter, and choose to correct the UltraVISTA fluxes to be consistent with the NMBS. We find very similar differences between the zero 

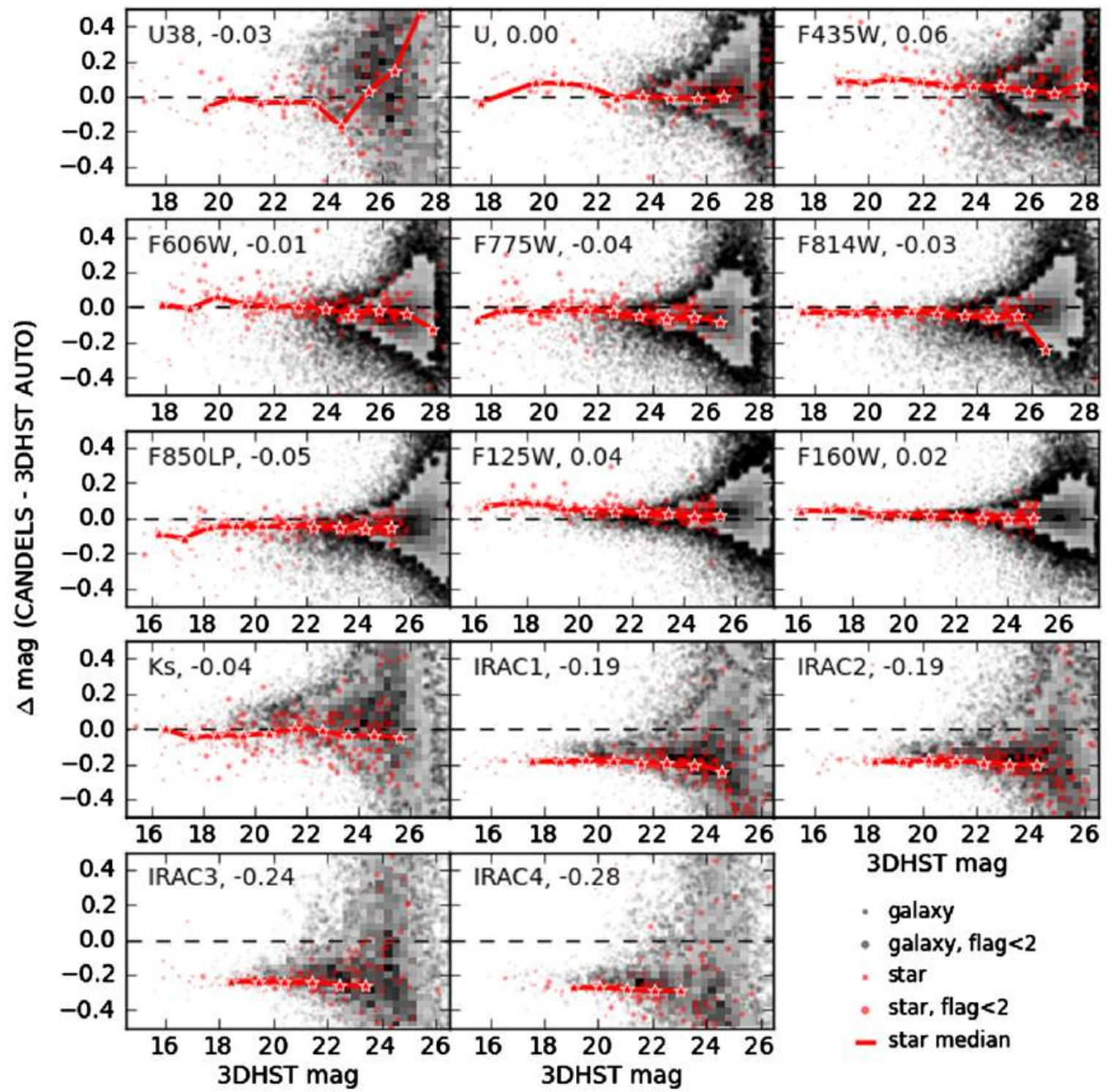

Figure 41. Comparison of the GOODS-S catalog AUTO fluxes to the CANDELS catalog total fluxes (Guo et al. 2013). Symbols are the same as in Figure 34. (A color version of this figure is available in the online journal.)

point corrections for the three $K$-bands $\left(\Delta K_{\mathrm{NMBS}}-K_{s \text {, WIRDS }}=\right.$ $\left.0.05 \mathrm{mag}, \Delta K_{\mathrm{NMBS}}-K_{s, \text { UVISTA }}=0.07 \mathrm{mag}\right)$. We have shifted all three bands fainter to agree with the (fainter) $H S T$ reference system, however.

\section{APPENDIX C}

\section{COMPARISONS TO OTHER CATALOGS}

In Figures 34-44 we show comparisons of each of our catalogs to other publicly available catalogs. We compare the AEGIS and COSMOS catalogs to their counterparts from the NEWFIRM Medium Band Survey (Whitaker et al. 2011). The GOODS-N catalog is compared to the Moircs Deep Survey (MODS) catalog from Kajisawa et al. (2011). There are a number of catalogs covering the GOODS-S field. Here we compare to the recent CANDELS catalog (Guo et al. 2013), the FIREWORKS catalog Wuyts et al. (2008) and the MUSYC survey (Cardamone et al. 2010), from which the medium band data are drawn. We compare the 3D-HST UDS catalog to the catalog published by Williams et al. (2009), an updated version of the same catalog, and the recent CANDELS catalog (Galametz et al. 2013).

A direct comparison of the aperture fluxes measured in two independent surveys is a useful diagnostic of problems with the photometry and catalog processing, particularly when the same images have been used and similar methods applied. However, where there are unresolved differences, it is not clear which catalog is more accurate. In this Appendix we aim to inform the reader what the differences between the 3D-HST and other available catalogs are, rather than commenting on the quality of either catalog. We therefore present comparisons of the default fluxes that would be used by anyone accessing the catalogs, rather than the fluxes from any intermediate stage, which may be more directly comparable. We note, for instance, that the fluxes in the CANDELS catalogs are more equivalent to our AUTO fluxes than our (default) total fluxes. Also, in the comparison to the NMBS we find better agreement before applying any zero point corrections than after, due to our choice of the HST filters as a reference for the zero point fitting. Where possible, we explain the offsets between the catalogs in the text. 


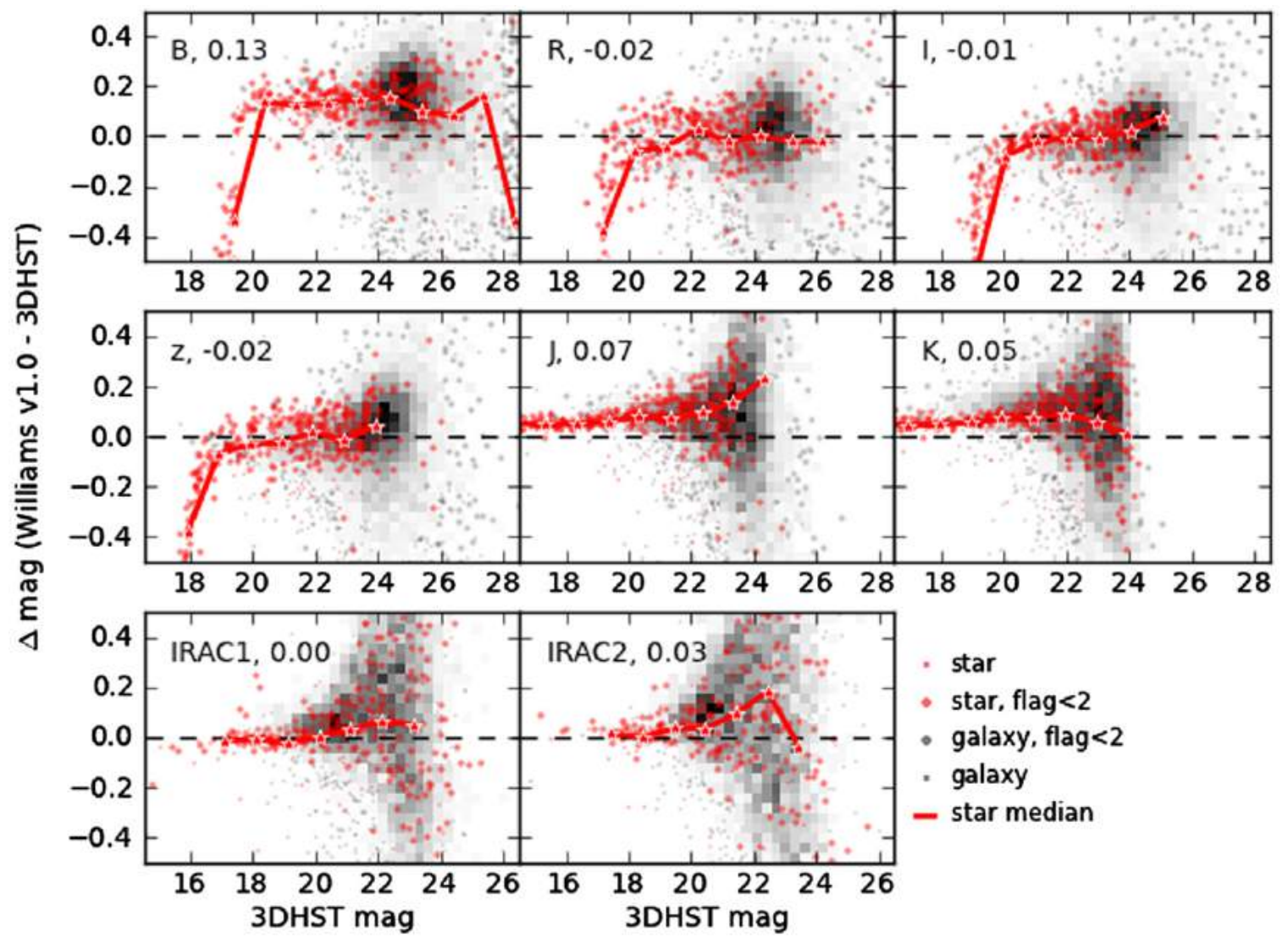

Figure 42. Comparison of the UDS catalog to the Williams et al. (2009) catalog. We compare total (rather than color aperture) fluxes from both catalogs. Symbols are the same as in Figure 34.

(A color version of this figure is available in the online journal.)

We compare the 3D-HST catalog total fluxes, which have been adjusted for zero point offsets, as described in Appendix B, to the total fluxes provided in each of the other catalogs. In some cases zero point offsets were calculated and applied in a similar way in the comparison catalogs. In the catalogs where color aperture fluxes and a correction to total in the detection band are provided, we convert the colors to total fluxes. In each panel we plot the difference between the total flux from the comparison catalog and the total flux in the 3D-HST catalog as a function of magnitude in that band from the 3D-HST catalog. We cross-match objects within $1^{\prime \prime}$ when the comparison catalog uses a ground-based detection image, and 0'.5 for the two WFC3detected CANDELS catalogs. The filter and median difference for stars is shown in the top left hand corner of each panel. The density of galaxies, selected with use_phot $=1$ is shown in the gray scale, with outlying objects shown as individual gray points. Stars (with star_flag =1) are shown in red. Objects that are not blended, with a SExtractor flag of $<2$, are shown by the larger points. Objects that have a flag $\geqslant 2$ are shown with small points. The median values for stars in bins of 1 magnitude are shown by the large red star symbols and the red solid line.

The AEGIS catalog is compared to the NMBS AEGIS v5.1 catalog (Whitaker et al. 2011) in Figure 34. The methods used for photometry in the two surveys are very similar and zero point corrections have been applied to both catalogs. The $U$-band measurements from the two surveys are in excellent agreement, although there is large scatter, and the same zero point offset of $-0.22 \mathrm{mag}$ was found in both cases. In the other bands, 3D-HST is fainter than the NMBS, with median offsets of 0.1-0.2 mag, and no significant trends with magnitude. The largest difference is $0.2 \mathrm{mag}$ in IRAC channel $4(8 \mu \mathrm{m})$. The offsets in the NIR can largely be explained by the zero point corrections applied to our catalogs in order to bring the ground-based data into agreement with the HST data. The (unadjusted) fluxes measured on the NMBS narrow-band $(J 1, J 2, J 3, H 1, H 2)$ and $K_{s^{-}}$ band images are in excellent agreement with the NMBS catalog fluxes, however we find that a shift of $\sim 0.15$ mag is necessary in order to bring them into agreement with the HST filters and the other ground-based NIR bands.

In Figures 35 and 36 we compare the COSMOS catalog to the NMBS v5.1 catalog (Whitaker et al. 2011). Zero point corrections have been applied to both catalogs. Here the offsets are larger on average but again constant with magnitude for most bands. The bands found to have the most uncertain zero points in NMBS ( $U$ and $I p$ ) have the largest offsets. For three of the medium bands and some of the optical bands, there is a turndown towards fainter magnitudes in 3D-HST for stars at bright end of the stellar sequence. These may be saturated stars for which the larger aperture (1..5) used in NMBS captures more of the light. The offsets in the NIR can largely be explained by the zero point corrections applied to both catalogs. If we remove the corrections from both and compare the measured photometry directly, we find differences of $\Delta$ (NMBS - 3DHST) $=-0.02$ to $-0.09 \mathrm{mag}$ for the bands between $z$ and $K$ in wavelength. Taking the NMBS $K$-band difference of $-0.03 \mathrm{mag}$ as a reference point would lead to differences between the bands of $-0.06 \mathrm{mag}$ (in the $J$-band) to $0.01 \mathrm{mag}$ (in $H 2$ ), both qualitatively and quantitatively similar to the actual zero point corrections applied within the NMBS catalog. We have also verified that the fluxes measured in large apertures on the NIR 

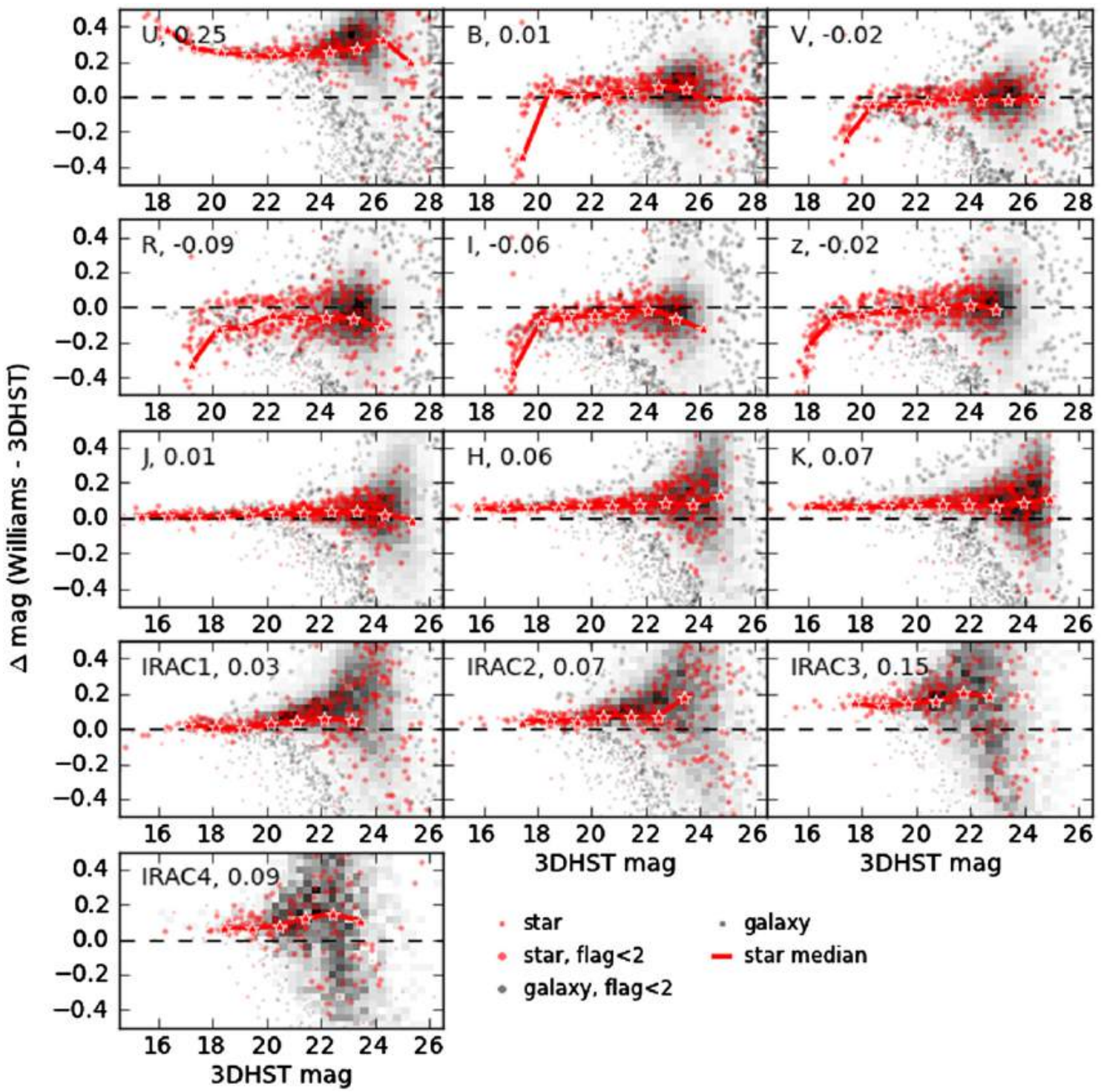

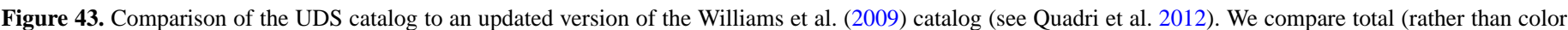

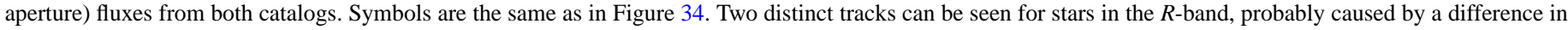

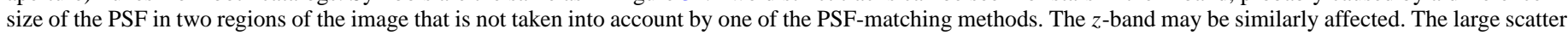
at the bright end in the other ground-based optical data suggests that there may also be (more subtle) variations in the PSF in these images.

(A color version of this figure is available in the online journal.)

NMBS images agree well with the total fluxes in the catalog and that the $H S T$-ground based colors measured in large apertures are consistent with the catalog colors. We are therefore confident that it is the zero points of the images themselves that are uncertain at this level rather than an error introduced by the aperture or total corrections applied in the analysis.

Figure 37 compares the GOODS-N catalog to the MODS Wide catalog (Kajisawa et al. 2011). The 1".2 aperture fluxes in the MODS catalog are converted to total using the ratio of the $K_{s}$-band MAG_AUTO_COR and aperture fluxes. There is large scatter between the fluxes in the Subaru optical bands, but fairly good agreement in the median. The NIR measurements agree well, with smaller scatter. There is a slight trend with magnitude, such that 3D-HST is brighter than MODS for fainter objects. The IRAC fluxes are brighter in 3D-HST, with an offset of $\sim 0.1$ mag in all four channels.

In Figure 38 we compare the total zero point corrected fluxes from the MUSYC Subaru v1.0 Catalog (Cardamone et al. 2010) to the 3D-HST fluxes. We have applied the recommended corrections to go from the MUSYC color aperture fluxes to galactic extinction and zero point corrected total magnitudes. The agreement for all bands is excellent. Outlying galaxies tend to be fainter in 3D-HST than MUSYC, however, with more objects are scattered below zero than above. There are also some differences for bright stars, with 3D-HST measuring lower fluxes at the very bright end than MUSYC.

Figure 39 compares the FIREWORKS catalog (Wuyts et al. 2008 ) to the 3D-HST GOODS-S catalog. We convert the color aperture fluxes in FIREWORKS to total using the ratio of the total to aperture flux in the $K_{s}$-band. The agreement is generally good in the optical and NIR. The $U$-band measurements are brighter by $0.18 \mathrm{mag}$ in 3D-HST, while the ACS $F 775 \mathrm{~W}$ and $F 850 L P$ bands are fainter by 0.09 mag. In the IRAC bands there are offsets of 0.13-0.21 mag, with FIREWORKS brighter than 3D-HST.

In Figure 40 we compare the 3D-HST GOODS-S catalog to the recent CANDELS catalog by Guo et al. (2013). The CANDELS fluxes in all the HST images are measured in an 


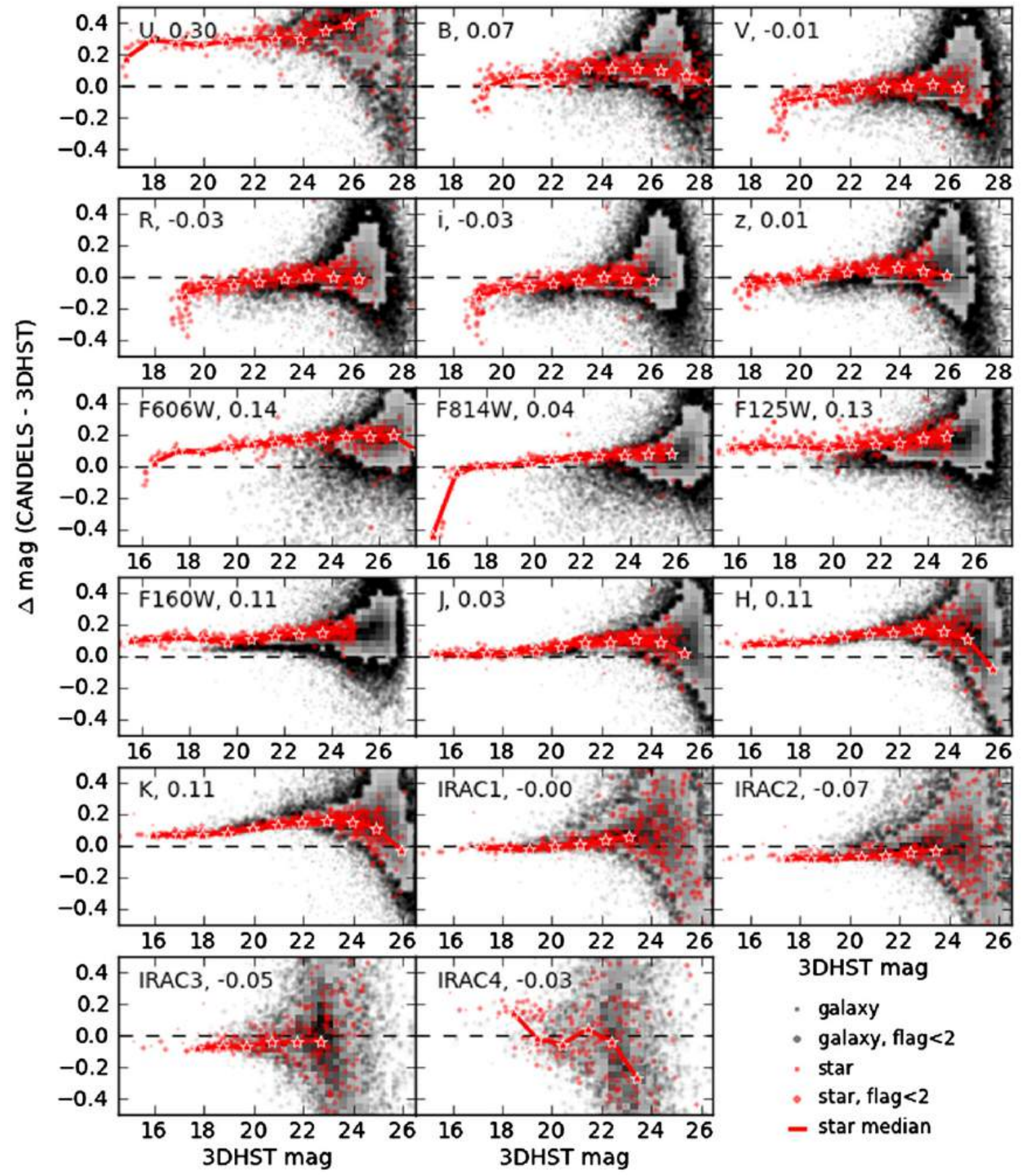

Figure 44. Comparison of the UDS catalog total fluxes to the CANDELS Galametz et al. (2013) catalog total fluxes. Symbols are the same as in Figure 34. The fluxes in the CANDELS catalog have not been corrected for the amount of light falling outside of the Kron radius, which accounts for the brighter total magnitudes in 3D-HST.

(A color version of this figure is available in the online journal.)

aperture given by the isophotal area of each source in the $F 160 \mathrm{~W}$ image. These "color" fluxes are then converted to total fluxes using the ratio of the AUTO and isophotal fluxes in $F 160 \mathrm{~W}$. The resulting total fluxes do not include a correction for the flux outside of the Kron radius and are fainter than the 3D-HST total fluxes by $\sim 0.05-0.15 \mathrm{mag}$ as a result. The offsets in the optical and NIR bands and the difference in the median for stars and galaxies are largely removed by comparing the 3D-HST AUTO fluxes to the CANDELS catalog fluxes. This comparison is shown in Figure 41. The IRAC fluxes measured by CANDELS using the TFIT software (Laidler et al. 2007), which applies similar techniques for the photometry of low-resolution images with a high-resolution prior, are $0.12-0.22$ mag brighter than the 3D-HST total fluxes.

Figure 42 compares the 3D-HST UDS catalog to the UDS v1.0 catalog from Williams et al. (2009). Figure 43 shows the comparison to an updated version of the same catalog, with significant updates as described by Quadri et al. (2012). The public catalog contains only 8 bands, with the newer catalog adding the $U, V$, and $H$-bands, and IRAC $5.8 \mu \mathrm{m}$ and $8 \mu \mathrm{m}$. The agreement with both catalogs is generally good, with large scatter in the $R$ and $z$-band, that separate more clearly into two tracks in the second $R$-band comparison. The $R$-band offsets show a spatial dependence, indicating that there may be a 

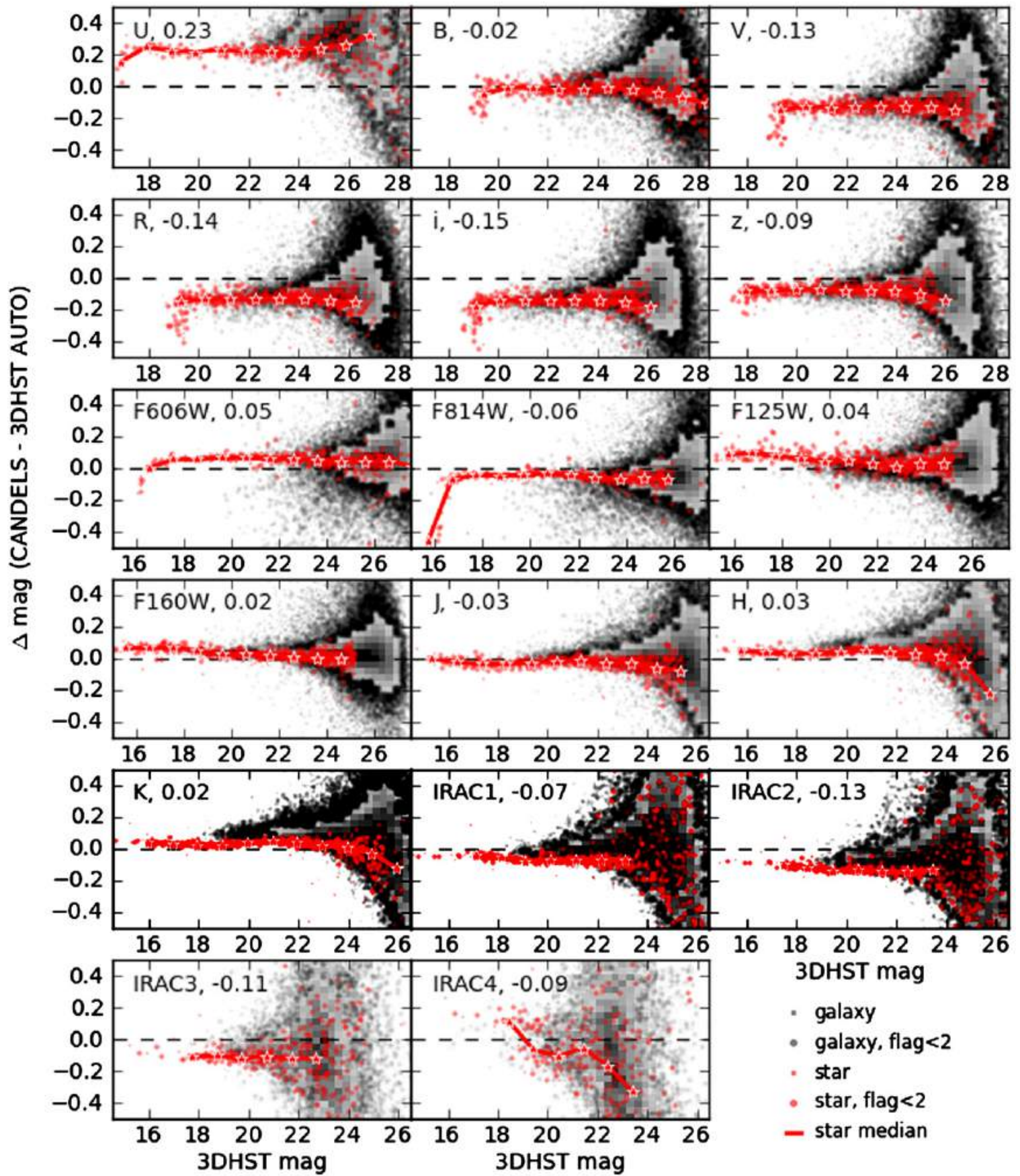

Figure 45. Comparison of the UDS catalog AUTO fluxes to the CANDELS Galametz et al. (2013) catalog total fluxes. Symbols are the same as in Figure 34.

(A color version of this figure is available in the online journal.)

systematic variation in the PSF or astrometry between two of the original SXDS fields that has not been accounted for in the mosaicking or by the photometry in one of the catalogs. Bright stars are systematically fainter in 3D-HST. There is a dependence on magnitude in the $I, J$ and IRAC bands in Figure 42 that is no longer visible in Figure 43. The $U$ band, for which the zero point is known to be uncertain, is offset brighter by 0.25 mag in $3 \mathrm{D}-\mathrm{HST}$.

Figure 44 compares the 3D-HST UDS catalog to the CANDELS catalog from Galametz et al. (2013). The methods applied for the CANDELS UDS catalog are very similar to those used for the Guo et al. (2013) catalog described above, and similar trends are seen in the comparisons. When we account for the correction from AUTO to total fluxes, which is not applied in the
Galametz et al. (2013) catalog, the offsets in the ACS and WFC3 bands largely disappear, as shown in Figure 45 . The median difference for stars decreases from 0.11 to $0.02 \mathrm{mag}$ in $F 160 \mathrm{~W}$, for example. With the exception of the $B$ band and NIR, the total fluxes measured on the low-resolution images agree better without adjusting for this difference, suggesting that the "dilation" method to grow the isophotal areas of objects before applying TFIT returns fluxes that are closer to the total flux measured by 3D-HST. We again find that the $U$-band is brighter in 3D-HST. There is large scatter in the IRAC4 $(8 \mu \mathrm{m})$ band.

\section{REFERENCES}

Adami, C., Mazure, A., Ilbert, O., et al. 2005, A\&A, 443, 805

Alexander, D. M., Bauer, F. E., Brandt, W. N., et al. 2003, AJ, 126, 539 
Ashby, M. L. N., Willner, S. P., Fazio, G. G., et al. 2013, ApJ, 769, 80 Barger, A. J., Cowie, L. L., \& Wang, W.-H. 2008, ApJ, 689, 687 Barmby, P., Huang, J.-S., Ashby, M. L. N., et al. 2008, ApJS, 177, 431 Bell, E. F., van der Wel, A., Papovich, C., et al. 2012, ApJ, 753, 167 Bertin, E., \& Arnouts, S. 1996, A\&AS, 117, 393

Bezanson, R., van Dokkum, P. G., van de Sande, J., et al. 2013, ApJL, 779, L21 Bielby, R., Hudelot, P., McCracken, H. J., et al. 2012, A\&A, 545, A23 Brammer, G. B., Sánchez-Janssen, R., Labbé, I., et al. 2012a, ApJL, 758, L17 Brammer, G. B., van Dokkum, P. G., \& Coppi, P. 2008, ApJ, 686, 1503 Brammer, G. B., van Dokkum, P. G., Franx, M., et al. 2012b, ApJS, 200, 13 Brammer, G. B., Whitaker, K. E., van Dokkum, P. G., et al. 2011, ApJ, 739, 24 Bruzual, G., \& Charlot, S. 2003, MNRAS, 344, 1000

Calzetti, D., Armus, L., Bohlin, R. C., et al. 2000, ApJ, 533, 682

Capak, P., Cowie, L. L., Hu, E. M., et al. 2004, AJ, 127, 180

Cardamone, C. N., van Dokkum, P. G., Urry, C. M., et al. 2010, ApJS, 189, 270

Casali, M., Adamson, A., Alves de Oliveira, C., et al. 2007, A\&A, 467, 777

Casertano, S., de Mello, D., Dickinson, M., et al. 2000, AJ, 120, 2747

Chabrier, G. 2003, PASP, 115, 763

Cirasuolo, M., McLure, R. J., Dunlop, J. S., et al. 2010, MNRAS, 401, 1166

Cohen, J. G. 2001, AJ, 121, 2895

Cohen, J. G., Hogg, D. W., Blandford, R., et al. 2000, ApJ, 538, 29

Cooper, M. C., Griffith, R. L., Newman, J. A., et al. 2012, MNRAS, 419, 3018

Cowie, L. L., Barger, A. J., Hu, E. M., et al. 2004, AJ, 127, 3137

Cuillandre, J.-C. J., Withington, K., Hudelot, P., et al. 2012, Proc. SPIE, 8448

Davis, M., Faber, S. M., Newman, J., et al. 2003, Proc. SPIE, 4834, 161

Davis, M., Guhathakurta, P., Konidaris, N. P., et al. 2007, ApJL, 660, L1

Dawson, S., Stern, D., Bunker, A. J., et al. 2001, AJ, 122, 598

Dickinson, M., Giavalisco, M., \& GOODS Team. 2003, in The Mass of Galaxies at Low and High Redshift, ed. R. Bender \& A. Renzini (Garching: ESO), 324

Erben, T., Hildebrandt, H., Lerchster, M., et al. 2009, A\&A, 493, 1197

Erben, T., Schirmer, M., Dietrich, J. P., et al. 2005, AN, 326, 432

Fabricant, D., Fata, R., Roll, J., et al. 2005, PASP, 117, 1411

Fioc, M., \& Rocca-Volmerange, B. 1997, A\&A, 326, 950

Fitzpatrick, E. L. 1999, PASP, 111, 63

Franx, M., van Dokkum, P. G., Schreiber, N. M. F., et al. 2008, ApJ, 688,770

Furusawa, H., Kosugi, G., Akiyama, M., et al. 2008, in ASP Conf. Ser. 399 Panoramic Views of Galaxy Formation and Evolution, ed. T. Kodama, T. Yamada, \& K. Aoki (San Francisco, CA: ASP), 131

Galametz, A., Grazian, A., Fontana, A., et al. 2013, ApJS, 206, 10

Gawiser, E., van Dokkum, P. G., Herrera, D., et al. 2006, ApJS, 162, 1

Geach, J. E., Simpson, C., Rawlings, S., et al. 2007, MNRAS, 381, 1369

Giavalisco, M., Ferguson, H. C., Koekemoer, A. M., et al. 2004, ApJL, 600, L93

Grogin, N. A., Kocevski, D. D., Faber, S. M., et al. 2011, ApJS, 197, 35

Guo, Y., Ferguson, H. C., Giavalisco, M., et al. 2013, ApJS, 207, 24

Hambly, N. C., Collins, R. S., Cross, N. J. G., et al. 2008, MNRAS, 384, 637

Hewett, P. C., Warren, S. J., Leggett, S. K., et al. 2006, MNRAS, 367, 454

Hildebrandt, H., Erben, T., Dietrich, J. P., et al. 2006, A\&A, 452, 1121

Hildebrandt, H., Pielorz, J., Erben, T., et al. 2009, A\&A, 498, 725

Hodgkin, S. T., Irwin, M. J., Hewett, P. C., et al. 2009, MNRAS, 394, 675

Hsieh, B.-C., Wang, W.-H., Hsieh, C.-C., et al. 2012, ApJS, 203, 23

Kajisawa, M., Ichikawa, T., Tanaka, I., et al. 2011, PASJ, 63, 379

Koekemoer, A. M., Faber, S. M., Ferguson, H. C., et al. 2011, ApJS, 197, 36

Koekemoer, A. M., Fruchter, A. S., Hook, R. N., et al. 2003, in HST Calibration Workshop: Hubble after the Installation of the ACS and the NICMOS Cooling System, ed. S. Arribas, A. Koekemoer, \& B. Whitmore (Baltimore, MD: Space Telescope Science Institute), 337

Kriek, M., van Dokkum, P. G., Labbé, I., et al. 2009, ApJ, 700, 221

Kron, R. G. 1980, ApJS, 43, 305

Kümmel, M., Walsh, J. R., Pirzkal, N., et al. 2009, PASP, 121, 59

Labbé, I., Bouwens, R., Illingworth, G. D., et al. 2006, ApJL, 649, L67

Labbé, I., Franx, M., Rudnick, G., et al. 2003, AJ, 125, 1107

Labbé, I., Huang, J., Franx, M., et al. 2005, ApJL, 624, L81
Laidler, V. G., Papovich, C., Grogin, N. A., et al. 2007, PASP, 119, 1325

Lawrence, A., Warren, S. J., Almaini, O., et al. 2007, MNRAS, 379, 1599

Lilly, S. J., Le Fèvre, O., Renzini, A., et al. 2007, ApJS, 172, 70

Lundgren, B. F., van Dokkum, P., Franx, M., et al. 2014, ApJ, 780, 34

Maraston, C. 2005, MNRAS, 362, 799

Marchesini, D., van Dokkum, P. G., Förster Schreiber, N. M., et al. 2009, ApJ, 701,1765

McCracken, H. J., Capak, P., Salvato, M., et al. 2010, ApJ, 708, 202

McCracken, H. J., Milvang-Jensen, B., Dunlop, J., et al. 2012, A\&A, 544, A156

Mink, D. J., Wyatt, W. F., Caldwell, N., et al. 2007, in ASP Conf. Ser. 376, Astronomical Data Analysis Software and Systems XVI, ed. R. A. Shaw, F. Hill, \& D. J. Bell (San Francisco, CA: ASP), 249

Muzzin, A., Marchesini, D., Stefanon, M., et al. 2013, ApJS, 206, 8

Nelson, E. J., van Dokkum, P. G., Brammer, G., et al. 2012, ApJL, 747, L28

Newman, J. A., Cooper, M. C., Davis, M., et al. 2013, ApJS, 208, 5

Nonino, M., Dickinson, M., Rosati, P., et al. 2009, ApJS, 183, 244

Oke, J. B. 1971, ApJ, 170, 193

Ono, Y., Ouchi, M., Shimasaku, K., et al. 2010, MNRAS, 402, 1580

Ouchi, M., Shimasaku, K., Akiyama, M., et al. 2008, ApJS, 176, 301

Papovich, C., Momcheva, I., Willmer, C. N. A., et al. 2010, ApJ, 716, 1503

Peng, C. Y., Ho, L. C., Impey, C. D., et al. 2010, AJ, 139, 2097

Pérez-González, P. G., Rieke, G. H., Villar, V., et al. 2008, ApJ, 675, 234

Pirzkal, N., Mack, J., Dahlen, T., et al. 2011, Sky Flats: Generating Improved

WFC3 IR Flat-fields, Tech. Rep. (Instrument Science Report WFC3-2011-

11; Baltimore, MD: Space Telescope Science Institute)

Quadri, R. F., Marchesini, D., van Dokkum, P., et al. 2007, AJ, 134, 1103

Quadri, R. F., Williams, R. J., Franx, M., et al. 2012, ApJ, 744, 88

Reddy, N. A., Erb, D. K., Steidel, C. C., et al. 2005, ApJ, 633, 748

Reddy, N. A., Steidel, C. C., Erb, D. K., et al. 2006, ApJ, 653, 1004

Retzlaff, J., Rosati, P., Dickinson, M., et al. 2010, A\&A, 511, A50

Rix, H.-W., Barden, M., Beckwith, S. V. W., et al. 2004, ApJS, 152, 163

Sanders, D. B., Salvato, M., Aussel, H., et al. 2007, ApJS, 172, 86

Schlafly, E. F., \& Finkbeiner, D. P. 2011, ApJ, 737, 103

Schlegel, D. J., Finkbeiner, D. P., \& Davis, M. 1998, ApJ, 500, 525

Scoville, N., Aussel, H., Brusa, M., et al. 2007, ApJS, 172, 1

Sérsic, J. L. 1968, Atlas de Galaxias Australes (Cordoba: Observatorio Astronomico)

Simpson, C., Martínez-Sansigre, A., Rawlings, S., et al. 2006, MNRAS, 372,741

Simpson, C., Rawlings, S., Ivison, R., et al. 2012, MNRAS, 421, 3060

Smail, I., Sharp, R., Swinbank, A. M., et al. 2008, MNRAS, 389, 407

Steidel, C. C., Adelberger, K. L., Shapley, A. E., et al. 2003, ApJ, 592, 728

Steidel, C. C., Shapley, A. E., Pettini, M., et al. 2004, ApJ, 604, 534

Szokoly, G. P., Bergeron, J., Hasinger, G., et al. 2004, ApJS, 155, 271

Tal, T., Dekel, A., Oesch, P., et al. 2014, ApJ, 789, 164

Taniguchi, Y., Scoville, N., Murayama, T., et al. 2007, ApJS, 172, 9

Treu, T., Ellis, R. S., Liao, T. X., et al. 2005, ApJL, 622, L5

Ueda, Y., Watson, M. G., Stewart, I. M., et al. 2008, ApJS, 179, 124

van Breukelen, C., Cotter, G., Rawlings, S., et al. 2007, MNRAS, 382, 971

van de Sande, J., Kriek, M., Franx, M., et al. 2013, ApJ, 771, 85

van der Wel, A., Bell, E. F., Häussler, B., et al. 2012, ApJS, 203, 24

van Dokkum, P. G., \& Brammer, G. 2010, ApJL, 718, L73

Whitaker, K. E., Labbé, I., van Dokkum, P. G., et al. 2011, ApJ, 735, 86

Whitaker, K. E., van Dokkum, P. G., Brammer, G., et al. 2012, ApJL, 754, L29

Whitaker, K. E., van Dokkum, P. G., Brammer, G., et al. 2013, ApJL, 770, L39

Williams, R. J., Quadri, R. F., Franx, M., et al. 2009, ApJ, 691, 1879

Windhorst, R. A., Cohen, S. H., Hathi, N. P., et al. 2011, ApJS, 193, 27

Wirth, G. D., Willmer, C. N. A., Amico, P., et al. 2004, AJ, 127, 3121

Wittman, D. 2009, ApJL, 700, L174

Wuyts, S., Förster Schreiber, N. M., Genzel, R., et al. 2012, ApJ, 753, 114

Wuyts, S., Labbé, I., Franx, M., et al. 2007, ApJ, 655, 51

Wuyts, S., Labbé, I., Schreiber, N. M. F., et al. 2008, ApJ, 682, 985

Yamada, T., Kodama, T., Akiyama, M., et al. 2005, ApJ, 634, 861

Yoshikawa, T., Akiyama, M., Kajisawa, M., et al. 2010, ApJ, 718, 112 